\title{
Les accidents d'irradiation Mise en place d'une base de données « ACCIRAD » à l'IPSN
}

\author{
V. CHAMBRETTE ${ }^{1}$, S. HARDY ${ }^{1}$, J.-C. NÉNOT ${ }^{1}$
}

(Manuscrit reçu le 3 mai 2001, accepté le $I^{e r}$ octobre 2001)

RÉSUMÉ Dans le cadre de ses missions d'expertise pour la protection de l'homme contre les rayonnements ionisants, l'IPSN a pour objectif de dresser un bilan des accidents d'origine radiologique survenus dans le monde ayant causé des effets déterministes afin d'en tirer les enseignements et d'établir un retour d'expérience. Une base documentaire informatique «ACCIRAD" de ces événements est établie en reprenant des données acquises à l'IPSN au cours de ses propres expertises, mais également des informations issues d'un travail de recherche bibliographique. Un recensement sous forme de tableau résumé est présenté dans cet article et une synthèse permettant d'établir un certain nombre de faits marquants a été réalisée. Dans un second temps, une consultation via Internet sera envisagée pour rendre accessible la base de données « ACCIRAD » au public intéressé.

ABSTRACT Radiological accidents - Database establishment "ACCIRAD" at IPSN.

One of the missions of the IPSN, as a part of expertise for human radiation protection, is to establish a review of radiological accidents resulting into deterministic effects occurring worldwide, in order to improve the management of radiation accident situations. For this purpose, a database called "ACCIRAD" has been created, gathering information obtained by the IPSN during its own expertise analyses or from data reported in the literature. A summary of the data is presented in a table in the present paper, as well as a global analysis of the accidental cases. $A$ consultation of "ACCIRAD" database by Internet will be possible, in the next future.

\section{Introduction}

Les accidents d'irradiation survenus et connus de par le monde restent des événements rares comparativement aux autres types d'accidents. Ils sont souvent peu documentés, qu'il s'agisse d'événements datant de plus de 10 ans (publications inexistantes) ou de ceux des dernières années (problème de confidentialité médicale). Or, il est essentiel de les recenser au mieux afin de tirer des enseignements importants sur les circonstances conduisant à une surexposition accidentelle, afin d'améliorer la prévention, la gestion de la situation de crise et les traitements médicaux appropriés.

\footnotetext{
I Institut de protection et de sûreté nucléaire, BP 6, 92265 Fontenay-aux-Roses Cedex, France.
} 
Dans le cadre de ses missions d'expertises médicales et sanitaires face aux expositions accidentelles aux rayonnements, l'IPSN dresse actuellement un bilan documentaire des accidents/incidents de surexposition connus depuis la Seconde Guerre mondiale et ayant causé des effets cliniquement observables. Pour cela, des données acquises par l'IPSN au cours de ses propres expertises ont été reprises. Par ailleurs, un travail de recherche bibliographique a été mené, et notamment une synthèse de différents recensements existant déjà dans la littérature a été effectuée (Lushbaugh et al., 1980 ; Dousset et Jammet, 1984 ; De Oliveira, 1987 ; Nénot, 1996 ; Verger et Winter, 1998 ; Cosset et al., 1999 ; Gonzales, 1999 ; Vargo, 1999 ; Croft et al., 1999 ; UNSCEAR, 2000a ; Gusev et al., 2001). Ces travaux présentent chacun leurs propres caractéristiques selon l'origine des données et l'objectif prédéfini ; ils sont souvent complémentaires. À partir de ces différents éléments, l'IPSN a mis en place deux actions. La première consiste à établir une base documentaire des principales publications faisant référence à ces événements. Dans le cadre de la deuxième, elle développe une base de données informatique baptisée «ACCIRAD ». Celle-ci présente 14 champs (date, pays, lieu, domaine de l'accident, catégorie d'accident, nature de la source, activité de la source, nombre d'exposés, nombre de blessés, nombre de décès, type d'exposition, informations cliniques, estimation de dose, références bibliographiques) renseignés pour chaque événement répertorié. Des recherches par tri selon un critère choisi sont réalisables et permettent aux utilisateurs de retrouver facilement les données de base de certains accidents.

Cet article a pour objet de présenter un certain nombre de faits marquants tirés de l'analyse des données recensées dans ACCIRAD, et d'adjoindre en annexe le bilan actuel des événements répertoriés par l'IPSN sous une forme réduite (extraction de 10 champs sur les 14 existants).

\section{Historique}

Dès la découverte des rayons $\mathrm{X}$ à la fin du $19^{\mathrm{e}}$ siècle, on peut identifier dans la littérature des événements accidentels d'irradiation de l'homme où sont relatés les effets néfastes des rayonnements ionisants, notamment sur la peau (De Oliveira, 1987). À partir des années 1940 et jusqu'en 1964, le développement des programmes de recherche et de mise au point de l'arme nucléaire provoquent une série d'accidents de criticité entraînant la mort de plus d'une dizaine de personnes (Charles, 2000). Dans la seconde moitié du siècle, le champ des applications des rayonnements ionisants s'étend au nucléaire civil et se diversifie de façon importante dans de nombreux secteurs. Les applications médicales et industrielles ont abouti à une multiplication des sources de petites dimensions, qui rend plus difficile leur suivi et la maîtrise de leur confinement. La figure 1 présente des 

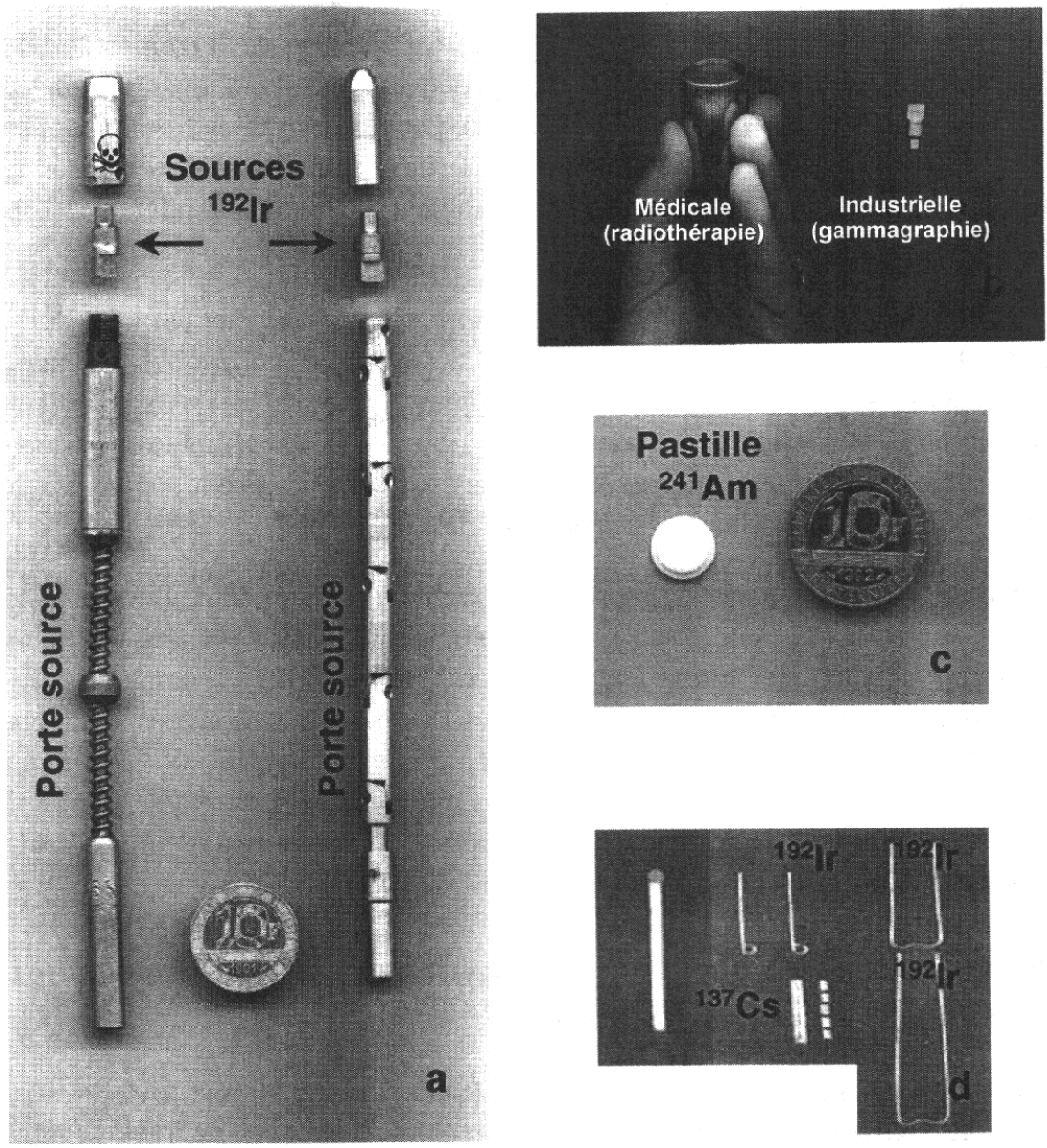

Figure 1 - Exemples de sources de rayonnements ionisants : (a) sources d'iridium 192 de gammagraphie industrielle avec leur porte source, (b) sources de cobalt 60 pour radiothérapie et gammagraphie industrielle, (c) pastille d'américium 241 pour la détection de fumée, (d) fils d'iridium 192 et de césium 137 pour la curiethérapie.

Examples of ionising radiation sources: (a) iridium 192 sources for industrial radiography with shielded housing, (b) cobalt 60 sources for medical radiotherapy and industrial radiography, (c) americium 241 source for smoke detector, (d) iridium 192 and caesium 137 needles for brachytherapy.

illustrations de sources de rayonnements ionisants utilisées pour différentes applications et montre leur taille et leur diversité. 


\section{CHAMBRETTE et al.}

Les raisons conduisant à une surexposition sont variées : non respect des règles élémentaires de radioprotection, manque de formation, erreur de jugement, erreur d'étalonnage... Dans bien des cas, la méconnaissance du danger retarde le diagnostic d'une surexposition accidentelle qui peut affecter dans certains cas un nombre important de personnes (Nénot, 1997). Ces accidents peuvent concerner toutes les catégories de population : professionnels, patients ou individus de la population générale.

Le nombre d'accidents dont l'origine radiologique est connue par hasard, est important. Il est donc possible d'imaginer que des accidents comparables aient pu passer inaperçus. C'est souvent l'état des victimes qui permet au médecin traitant d'évoquer la cause radiologique. À ce propos, il est permis de s'interroger sur le nombre d'accidents graves méconnus, dont les conséquences sont attribuées, du moins pour les plus anciens, à des causes banales.

La base ACCIRAD, mise à jour régulièrement, établit à partir de l'année 1945 le recensement des accidents/incidents radiologiques connus. Plus de cinq cent événements de surexposition aux rayonnements ionisants ont été ainsi recensés depuis cette date jusqu'à la fin de 2000 (Tab. I en annexe). Ce sont des situations qui ont entraîné une exposition de personnes à des doses supérieures aux limites réglementaires et ont souvent causé des effets cliniquement observables, voire le décès des victimes. Tous ces événements répertoriés sont répartis uniformément tout au long des années.

\section{Accidents de surexposition aux rayonnements ionisants ayant entraîné le décès des victimes}

Sur les 560 événements recensés dans ACCIRAD, 70 ont entraîné le décès d'au moins une victime. Ces accidents radiologiques ont provoqué au total la mort d'environ 180 personnes consécutivement à un syndrome aigu d'irradiation.

La figure 2 présente depuis 1945 et au fil des années, le nombre de victimes décédées au cours des accidents mortels. Parmi ceux-ci, 7 accidents ont été particulièrement dramatiques :

- deux accidents militaires russes de sous-marins (en 1961, avec 8 morts et en 1985, avec environ 10 morts) ;

- deux accidents liés à la perte d'une source industrielle (en 1982, à Bakou avec 5 morts et en 1984, à Casablanca avec 8 morts) ;

- deux accidents de radiothérapie (en 1990, à Saragosse, et en 1996, à San José avec au moins 13 morts dans chacun des deux cas);

- l'accident de Tchernobyl, en 1986, avec 28 morts. 


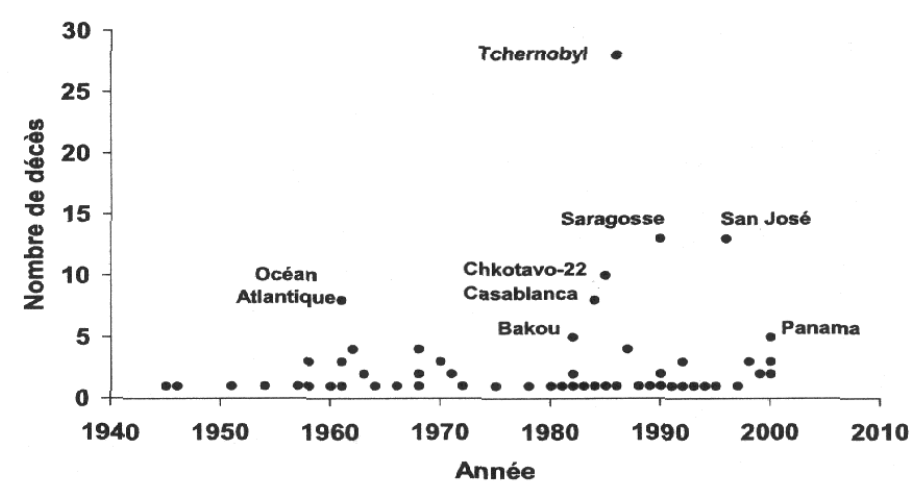

Figure 2 - Victimes décédées depuis 1945, au cours d'accidents radiologiques.

Number of death from accidental overexposure since 1945.

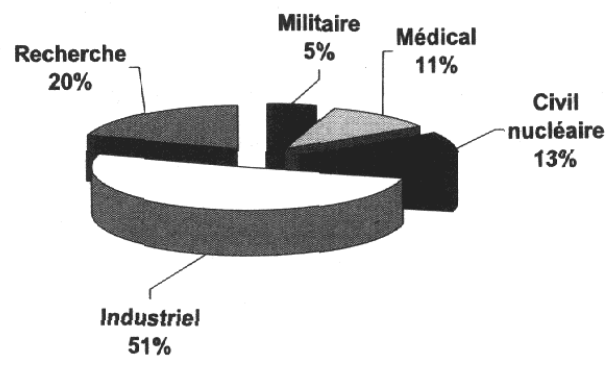

Figure 3 - Répartition des accidents radiologiques suivant les différents secteurs d'activité, depuis 1945.

Distribution of radiological accidents according to different sectors since 1945.

Dans les autres cas, le nombre de victimes reste chaque fois inférieur à 5 personnes.

\section{Accidents radiologiques dans les différents secteurs d'activité}

Depuis la multiplication des champs d'application des rayonnements ionisants ces cinquante dernières années, les accidents radiologiques ne concernent plus seulement les applications militaires mais aussi le nucléaire civil, l'industrie, le monde médical et celui de la recherche. La figure 3 présente la répartition suivant les 


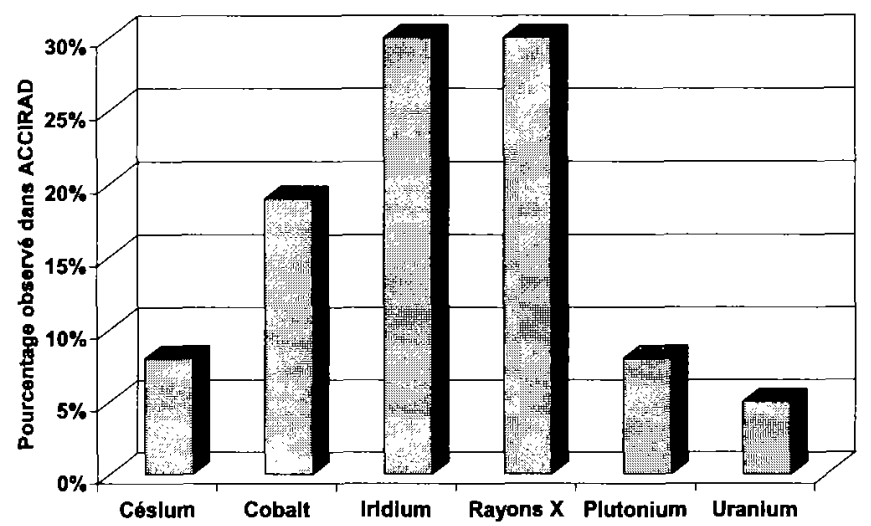

Figure 4 - Répartition des principales sources de rayonnements ionisants à l'origine des surexpositions accidentelles, depuis 1945.

Distribution of accidental overexposures according to the origin of the source since 1945.

différents secteurs d'activité à partir des données de ACCIRAD. Ce graphe met en évidence la prédominance de l'origine industrielle des accidents radiologiques: $51 \%$ des situations accidentelles sont liées au secteur industriel contre $20 \%$ à celui de la recherche, $13 \%$ à celui du nucléaire civil et $11 \%$ au celui de la médecine.

\section{Types de sources à l'origine des accidents radiologiques}

Si l'on s'intéresse plus particulièrement au type de sources qui sont à l'origine des surexpositions, la base ACCIRAD montre qu'elles peuvent être très variées : des sources scellées d'iridium et de cobalt, des rayonnements X d'appareil de mesure ou encore des produits de fission en milieu civil nucléaire, mais aussi des sources plus hétéroclites (curium 242, iode 125, indium 110, polonium 210). La figure 4 présente, en se basant sur les 560 événements recensés dans ACCIRAD, les principales sources responsables des surexpositions accidentelles et leur répartition en pourcentage. Les sources de rayons $\mathrm{X}$, d'iridium et de cobalt sont les plus souvent mises en cause et concernent respectivement $30 \%, 30 \%$ et $19 \%$ des situations accidentelles.

\section{Accidents radiologiques dans le secteur industriel}

Dans le secteur industriel, les sources d'iridium arrivent en tête et concernent près de $50 \%$ des cas (Fig. 5). Dans ce cas, ce sont surtout des surexpositions liées à une mauvaise utilisation des appareils de gammagraphie utilisés pour le contrôle des 


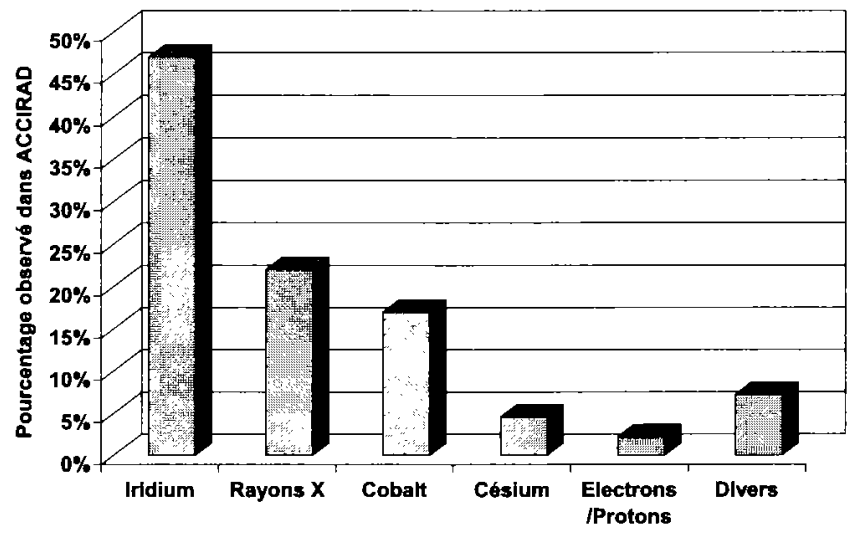

Figure 5 - Répartition des sources de rayonnements ionisants à l'origine des surexpositions accidentelles dans le secteur industriel, depuis 1945.

Percentages of accidental overexposures in industries according to the origin of the source since 1945.

soudures. Cependant, pour relativiser ces chiffres, il faut noter que ces opérations de contrôle sont maintenant très fréquentes dans le secteur industriel et que le nombre d'accidents occasionnés par de telles sources reste faible quand il est comparé au nombre de sources disponibles : en France, selon les données établies par la Commission interministérielle des radioéléments artificiels (CIREA), on dénombre 30000 sources industrielles (tous types confondus) parmi lesquelles sont disponibles et utilisées 800 sources de gammagraphie. Cependant, aucun accident radiologique lié à l'usage de ces sources n'a été enregistré depuis 1979, en France. Au sujet des accidents de gammagraphie, l'Agence internationale de l'énergie atomique (AIEA) a rédigé une synthèse intéressante sur les leçons tirées des circonstances ayant conduit à de telles surexpositions (IAEA, 1998). Les opérations de mesure à l'aide d'appareils émetteurs de rayons $\mathrm{X}$ occasionnent $22 \%$ des surexpositions dans le domaine industriel, en provoquant en général des irradiations localisées. Dans le cas des irradiateurs industriels au cobalt, le non respect des règles de sécurité, notamment dans certaines opérations de maintenance, conduit dans $17 \%$ des cas à une irradiation externe par les rayonnements gamma.

\section{Accidents radiologiques dans le secteur médical}

Parmi les 560 événements répertoriés, plus de $10 \%$ d'entre eux ont eu lieu dans le domaine médical. Les événements concernent essentiellement la radiothérapie 


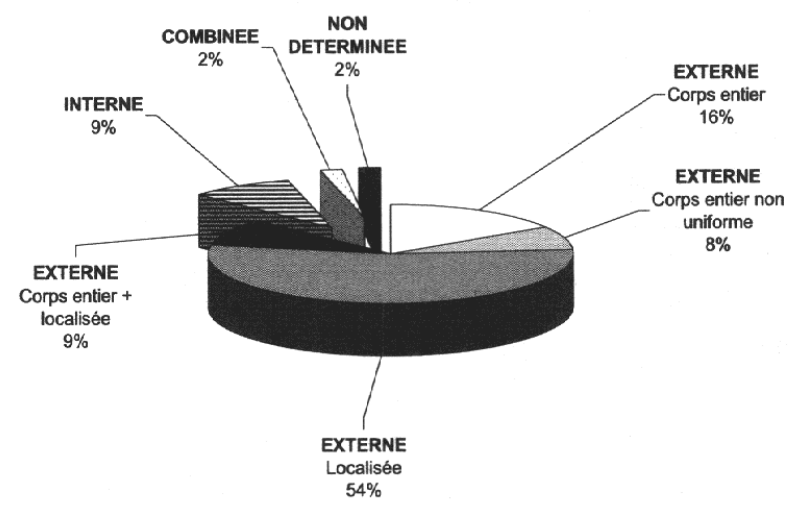

Figure 6 - Répartition des différents types d'exposition au cours des accidents d'irradiation. Distribution of different types of exposures from accidental accidents.

et la curiethérapie. On dénombre parmi ceux-ci également un certain nombre d'accidents dont l'origine est la perte d'une source. Dix-sept de ces accidents liés au domaine médical ont entraîné le décès d'au moins 49 personnes consécutivement à un syndrome aigu d'irradiation. Ces incidents/accidents ont principalement impliqué des patients et dans une moindre mesure, le personnel médical ainsi que le public quand une source d'origine médicale fut égarée. Ces événements mettent en évidence des erreurs de traitement (inversion de patient, mauvais ciblage d'organe, confusion de radionucléide injecté, mauvais étalonnage de la source, calcul de dose erroné) mais également des erreurs techniques (erreur d'utilisation ou déficience d'un appareillage). À partir d'une centaine de cas, l'AIEA décrit précisément les circonstances, les conséquences médicales et les leçons tirées d'erreurs de manipulation des rayonnements ionisants (IAEA, 2000). Aucun type d'accident ne prédomine dans le secteur médical que ce soit en considérant le type de source impliquée, ou les circonstances ayant conduit à la surexposition.

\section{Différents types de surexposition}

Une autre analyse de l'ensemble des données répertoriées dans ACCIRAD consiste à classer les accidents selon le type d'exposition qu'ils ont entraîné sur les victimes : externe global, externe non uniforme (propre aux accidents de criticité), externe localisé, interne, combiné externe et interne. La figure 6 illustre la répartition de ces différents modes d'agression des rayonnements ionisants. Parmi les événements recensés, $87 \%$ des accidents concernent des irradiations de type 
purement externe. La part des accidents/incidents par voie interne (contamination) est certainement sous-évaluée ( $11 \%$ ) car leur inventaire est avant tout difficile à établir (données non régulièrement transmises quand elles concernent les travailleurs du nucléaire). De plus, les cas de contamination interne ont rarement des conséquences médicales sévères, et la base ACCIRAD recense les événements causant des effets déterministes. Il est intéressant de noter que la moitié des surexpositions externes sont de type localisé (54\%): elles concernent des opérations de mesure (gammagraphie, spectrométrie...) et aussi des accidents dus à la perte et récupération de sources. Dans ce cas, on peut observer des brûlures radio-induites « multifocales ».

Les surexpositions externes globales ou localisées ont concerné plus de 15000 personnes depuis 1945. La part représentée par les accidents d'irradiation localisée est importante, mais ceux-ci affectent peu de personnes au cours de l'événement. Cette observation doit néanmoins être pondérée par le fait qu'un grand nombre de tels accidents restent méconnus car trop souvent attribués à d'autres causes. Inversement, les circonstances des accidents de surexposition du corps entier entraînent souvent l'irradiation de plusieurs personnes.

\section{Conclusions}

Cette base de données met en lumière quatre évidences.

Les accidents d'irradiation restent des événements rares. La comparaison du registre ACCIRAD avec les accidents d'autre nature montre que leur nombre total et le nombre de victimes par accident sont petits. Dans le monde entier (mis à part les grandes catastrophes naturelles, les accidents de transports routiers et aériens), les événements qui causent un grand nombre de victimes sont les naufrages de ferry-boats (environ 5000 noyés entre 1990 et 2000), les explosions d'oléoducs ou de minéraliers transportant le gaz et le pétrole (un millier de morts au cours de la même période), les ruptures de barrage (plus de 2000 morts dans les années 50 et 60 en Europe) et les mouvements de foule (plusieurs dizaines de morts à chaque accident dans le métro, les stades de Football, etc.).

La plupart de ces accidents auraient pu être prévenus et évités, ou tout au moins leurs conséquences largement amoindries, si les règles élémentaires de sécurité et le respect des consignes avaient été observés.

Les petites sources mobiles causent beaucoup plus d'accidents que les grosses sources confinées. Deux accidents graves de réacteurs nucléaires, avec destruction totale de l'installation (Winscale au Royaume-Uni en 1957 et TMI aux USA en 1979), n'ont causé aucune victime. L'accident de Tchernobyl en 1996 a 
fait 28 victimes parmi les intervenants. De plus, il a entraîné des effets stochastiques non pris en compte dans ACCIRAD : le bilan 2000, qui risque de s'alourdir, fait état de 1800 cancers de la thyroïde chez les enfants (UNSCEAR, $2000 \mathrm{~b}$; Verger et al., 2000). En cinquante ans, les accidents mortels dus à des irradiateurs industriels sont au nombre de quatre, chacun causant un unique décès. Dans le même temps, les petites sources, particulièrement celles destinées à la gammagraphie, ont causé 13 morts et entraîné des séquelles graves chez une quarantaine de personnes.

\section{Les accidents sont beaucoup plus fréquents dans le domaine industriel que} dans le domaine médical. Cependant, s'il y a peu d'accidents qui surviennent en milieu médical (particulièrement quand le nombre d'accidents est rapporté au nombre de malades traités par les rayonnements ou, encore mieux, au nombre d'actes de radiologie et de radiothérapie), le nombre de victimes par accident est souvent plus élevé que dans l'industrie. Ceci est dû au fait que, très souvent, l'erreur n'est reconnue qu'à l'apparition des signes patents de la surexposition, après qu'un certain nombre de patients ait été traités par l'appareil défectueux.

Enfin, il faut bien reconnaître que cette base documentaire ne relève que les événements mentionnés dans la littérature. Ceci pose la question du nombre d'accidents graves inconnus, soit parce que leur lien avec les rayonnements ionisants n'a pas été établi, soit qu'ils n'ont pas été révélés à la communauté internationale. Il existe sans nul doute des accidents militaires qui ont été volontairement cachés, particulièrement durant la guerre froide. La volonté de transparence des autorités politiques de la plupart des pays a grandement contribué à la connaissance de ce genre d'accidents, mais le nombre exact est sans doute encore en dessous des annonces officielles. Il est cependant vraisemblable qu'aujourd'hui, la fraction ignorée des accidents avec conséquences sévères (morts, blessures graves ou grand nombre de victimes), est petite par rapport à la partie connue. Le bilan est certainement beaucoup moins complet pour les accidents qui ont entraîné des conséquences relativement mineures au plan clinique. En effet, dans beaucoup de pays (développés ou non) il n'est pas tenu de registre particulier et les accidentés sont orientés vers des services hospitaliers spécialisés divers (chirurgie réparatrice, grands brûlés, dermatologie, orthopédie, etc.) ; de ce fait, les bilans nationaux sont difficiles à établir, surtout s'il n'existe pas de volonté politique et scientifique. Il faut aussi tenir compte des accidents bénins qui n'entraînent aucune traduction pathologique au-dessous de certains niveaux de dose. Ceci favorise leur ignorance et fait que le nombre réel d'accidents mineurs, mais qui répondent néanmoins à la définition choisie par ACCIRAD, est beaucoup plus important que celui relevé. 
Dans un second temps, l'IPSN envisage d'implanter cette base sur son site Internet afin d'en rendre ses données facilement accessibles. Cette initiative devrait favoriser l'échange des informations sur le recensement des accidents radiologiques, et ainsi améliorer l'exactitude des données de la base ACCIRAD.

\section{RÉFÉRENCES}

Charles T. (2000) Les accidents de criticité dans le monde, Colloque Nucléaire et Santé : Actualités 99 , 28 janvier 2000 , Paris.

Cosset J.M., Perdereau B., Dubray B., Helfre S., Brixy F., Gongora R., Fourquet A. (1999) The 19511998 experience of Paris Institute Curie Radiopathology Unit: a preliminary report, J. Radiol. Prot. 19 (4), 293-304.

Croft J.R., Thomas G.O., Walker S., Williams C.R. (1999) IRID: ionising radiations incident database, First review of cases reported and operation of database, NRPB.

De Oliveira A.R. (1987) Un répertoire des accidents radiologiques, 1945-1985, Radioprotection 22 (2), 89-135.

Dousset M., Jammet H. (1984) Les accidents humains d'irradiation d'origine nucléaire. Dans : Irradiations accidentelles et thérapeutiques (P. Galle, R. Masse, J.C. Nénot, Eds) pp. 27-61. Masson, Paris.

Gonzales A.J. (1999) Des initiatives qui s'imposent, Bull. IAEA 41 (3), 2-17.

Gusev I.A, Guskova A.K., Mettler F.A. Eds (2001) Medical management of radiation accidents, 2nd edn. CRC Press, Boca Raton, London, New York, Washington. D.C.

IAEA (1998) Lessons learned from accidental exposures in industrial radiography, Safety Reports Series No. 7, Vienna.

IAEA (2000) Lessons learned from accidental exposures in radiotherapy, Safety Reports Series No. 17, Vienna.

Lushbaugh C.C., Fry S.A., Hübner K.F., Ricks R.C. (1980) Total-body irradiation: A historical review and follow-up. Dans: The medical basis for radiation accident preparedness (K.F. Hübner, S.A. Fry, Eds) pp. 3-15. Elsevier, New York.

Nénot J.C. (1996) Radiation Accidents, Rad. Prot. Dos. 68 (1/2), 1 1 1-118.

Nénot J.C. (1997) La gestion sanitaire des accidents radiologiques et nucléaires : leçons du passé, SFRP 97, Congrès national de radioprotection, Avignon, 18-20 juin 1997, Actes, 137-145, SFRP Ed.

UNSCEAR (2000a) Sources and effects of ionizing radiation, Report to the general assembly with scientific annexes, I (annexe E), 638-645.

UNSCEAR (2000b) Sources and effects of ionizing radiation, Report to the general assembly with scientific annexes, II (annexe J), 453-566.

Vargo G.J. (1999) A brief history of nuclear criticality accidents in Russia: 1953-1997, Health Phys. 77 (5), 505-511.

Verger P., Winter D. (1998) Radiation Accidents. Dans : Encyclopaedia of Occupational Health and Safety (O.M.S. Eds) pp. 39.29-39.28.

Verger P., Cherie-Challine L., Boutou O., Champion D., Gourmelon P., Hubert Ph., Isnard H., Joly J., Jouan M., Pirard Ph., Renaud Ph., Tirmarche M., Vidal M. (2000) Évaluation des conséquences sanitaires de l'accident de Tchermobyl en France : dispositif de surveillance épidémiologique, état des connaissances, évaluation des risques et perspectives, Rapport IPSN-InVS, Réf. IPSN/00-15a. 


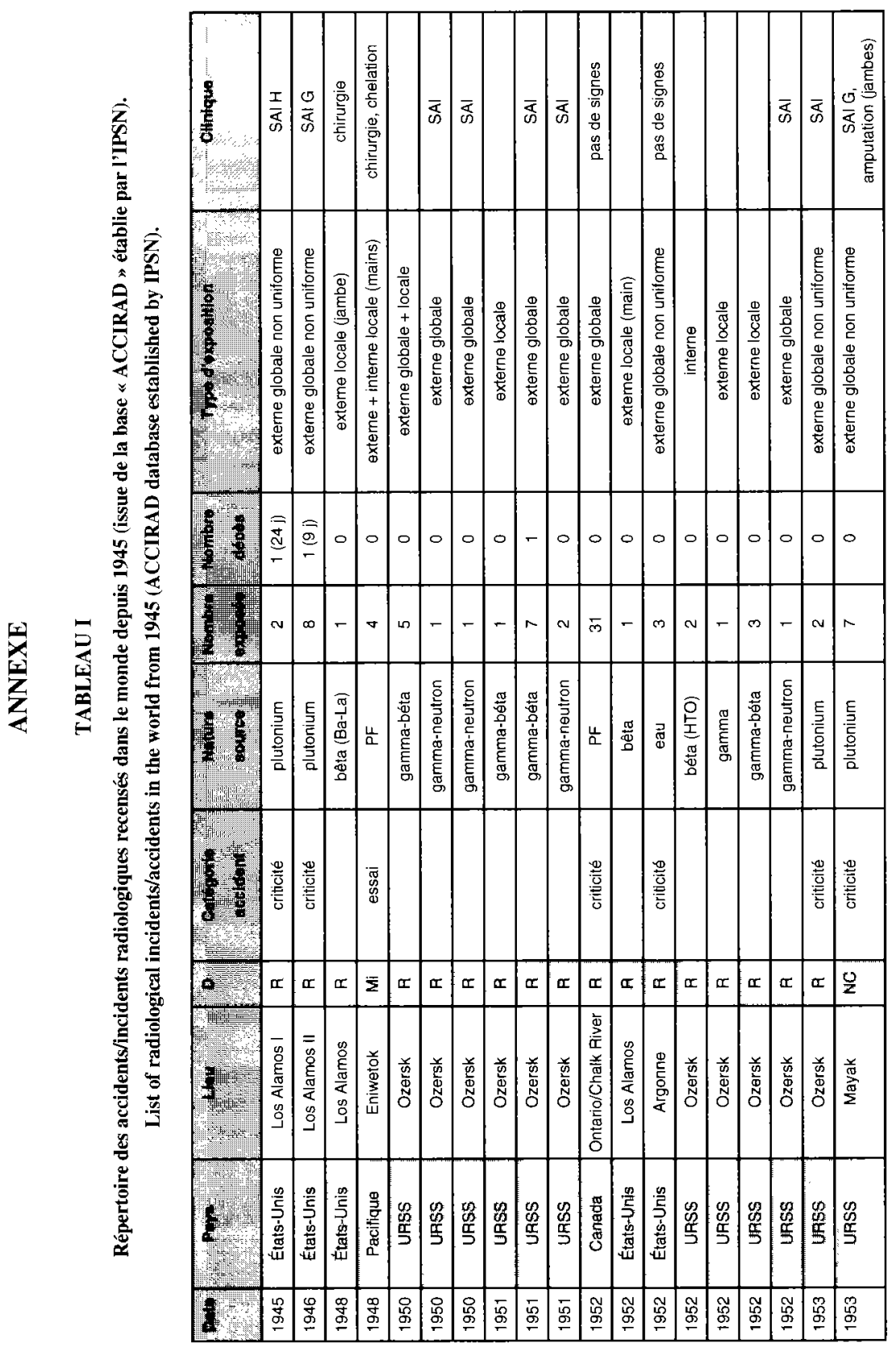




\begin{tabular}{|c|c|c|c|c|c|c|c|c|c|c|c|c|c|c|c|c|c|c|c|c|c|c|c|c|c|}
\hline 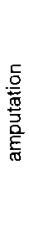 & & $\bar{\sigma}$ & 芯 & के & $\bar{\xi}$ & 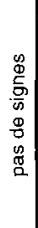 & & $\begin{array}{l}\bar{\sigma} \\
0\end{array}$ & $\bar{\alpha}$ & $\overline{\mathbb{a}}$ & 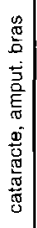 & 衣 & & & 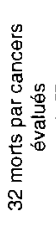 & & 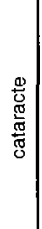 & 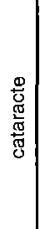 & & & & 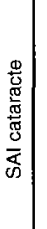 & & 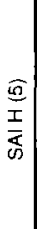 & 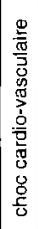 \\
\hline 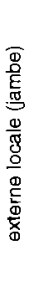 & $\begin{array}{l}\stackrel{\Phi}{E} \\
\stackrel{\Phi}{\Phi} \\
\underline{\underline{E}}\end{array}$ & 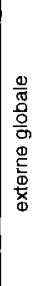 & 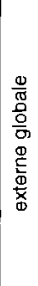 & 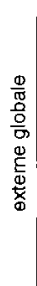 & 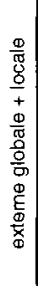 & 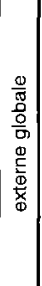 & 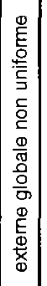 & 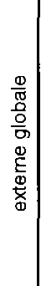 & 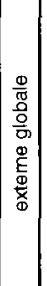 & 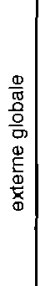 & 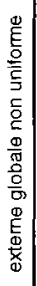 & 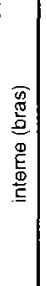 & 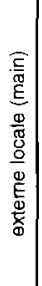 & 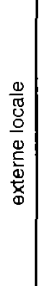 & 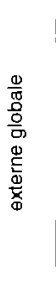 & 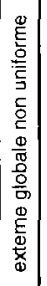 & 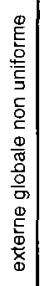 & 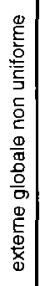 & 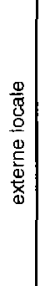 & 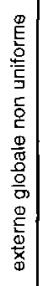 & 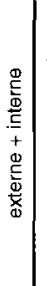 & 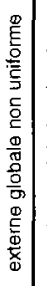 & 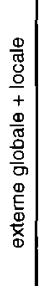 & 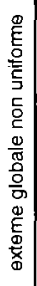 & 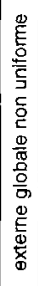 \\
\hline 0 & 0 & $\circ$ & 0 & 0 & 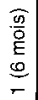 & 0 & 0 & - & 0 & 0 & $\circ$ & 0 & 0 & 0 & 0 & $\circ$ & 0 & 0 & 0 & 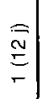 & c. & 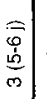 & $\circ$ & 0 & $\begin{array}{l}\widehat{I} \\
\stackrel{5}{0} \\
\stackrel{9}{\sigma}\end{array}$ \\
\hline- & $\mathrm{N}$ & $\infty$ & in & $\sigma$ & 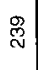 & $\omega$ & 오 & $\infty$ & - & $\sim$ & - & - & - & - & 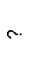 & $\sim$ & - & - & - & $\sigma$ & $\begin{array}{l}8 \\
\square \\
\mp\end{array}$ & 寸 & $\stackrel{m}{-}$ & $\infty$ & on \\
\hline 8 & $\begin{array}{l}\frac{0}{0} \\
\frac{5}{1} \\
\frac{5}{5} \\
\frac{9}{8}\end{array}$ & 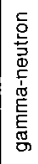 & 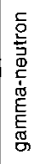 & 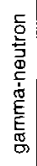 & 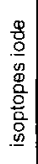 & 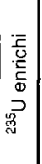 & 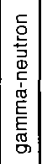 & 웅 & 点 & 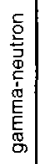 & & 票 & 票 & $\stackrel{8}{8}$ & $\frac{\Delta}{\alpha}$ & & & & 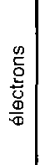 & 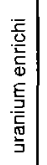 & 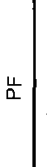 & 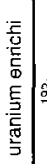 & $\stackrel{2}{\alpha} \underset{\alpha}{\alpha}$ & 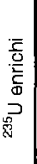 & 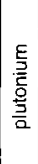 \\
\hline & & & & & $\begin{array}{l}\cdot \bar{\nabla} \\
\bar{y} \\
0 \\
\Phi\end{array}$ & 孪 & 离 & 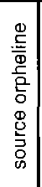 & 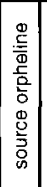 & 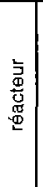 & 恋 & 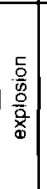 & & 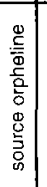 & 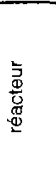 & 离 & $\frac{\mathscr{D}}{\frac{D}{\underline{\underline{T}}}}$ & : & 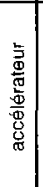 & 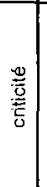 & 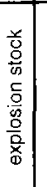 & $\begin{array}{l}\frac{\Phi}{\mathrm{O}} \\
\frac{\mathrm{E}}{\mathrm{E}}\end{array}$ & 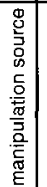 & 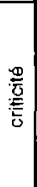 & 䍝 \\
\hline- & $\alpha$ & $\mathrm{O}$ & $\underline{z}$ & $\frac{O}{2}$ & $\bar{\Sigma}$ & 도 & 피 & $\bar{\Sigma}$ & $\cdots$ & $\underline{z}$ & 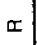 & - & 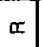 & - & $\mathcal{U}$ & 따 & 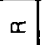 & $x$ & $\widetilde{I}$ & $\frac{U}{z}$ & $\bar{\Sigma}$ & $\mathbb{Z}$ & a. & $\mathcal{U}$ & $\mathcal{O}$ \\
\hline 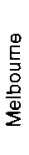 & 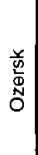 & $\begin{array}{l}\frac{.}{2} \\
\text { 荝 } \\
\stackrel{N}{N} \\
0\end{array}$ & $\begin{array}{l}\frac{\omega}{5} \\
\frac{5}{N} \\
0 \\
0\end{array}$ & 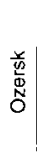 & 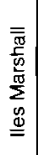 & 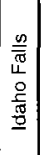 & 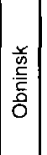 & 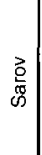 & $\begin{array}{l}\vec{z} \\
\stackrel{0}{0} \\
0 \\
\stackrel{0}{\Sigma}\end{array}$ & 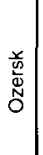 & & $\begin{array}{l}\frac{\text { 몽 }}{\frac{9}{5}} \\
\frac{\pi}{1}\end{array}$ & 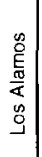 & 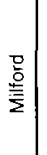 & 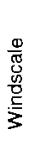 & & & & $\begin{array}{l}\vec{z} \\
\text { o. } \\
\text { o. } \\
\Sigma\end{array}$ & $\frac{\sqrt{0}}{\stackrel{a}{a}}$ & $\begin{array}{l}\underset{\frac{E}{5}}{D} \\
\frac{2}{2} \\
\frac{2}{2}\end{array}$ & $\frac{\sqrt{0}}{\stackrel{m}{\pi}}$ & 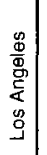 & 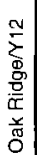 & $\begin{array}{l}\equiv \\
\equiv \\
0 \\
0 \\
\frac{\pi}{\pi} \\
\frac{\pi}{0} \\
0 \\
0\end{array}$ \\
\hline 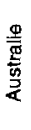 & 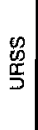 & $\begin{array}{l}\mathscr{O} \\
\stackrel{0}{\leftrightarrows}\end{array}$ & 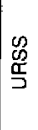 & $\begin{array}{l}\text { क } \\
\frac{5}{5} \\
\frac{5}{5}\end{array}$ & 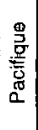 & 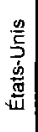 & $\begin{array}{l}\text { D } \\
\stackrel{\mathscr{C}}{5}\end{array}$ & $\begin{array}{l}\mathscr{0} \\
\stackrel{2}{5} \\
\stackrel{5}{5}\end{array}$ & $\begin{array}{l}\mathscr{W} \\
\mathscr{D} \\
\stackrel{5}{5}\end{array}$ & $\begin{array}{l}\mathscr{0} \\
0 \\
\frac{2}{5}\end{array}$ & $\begin{array}{l}\stackrel{\infty}{\mathscr{2}} \\
\text { 苑 }\end{array}$ & 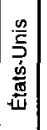 & 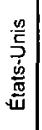 & 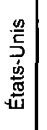 & 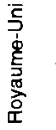 & $\begin{array}{l}\mathscr{\infty} \\
\stackrel{5}{5} \\
5\end{array}$ & $\begin{array}{l}0 \\
0 \\
0 \\
\frac{0}{5}\end{array}$ & $\begin{array}{l}\infty \\
0 \\
\frac{\pi}{5}\end{array}$ & $\begin{array}{l}\mathscr{0} \\
\mathscr{0} \\
\frac{5}{5}\end{array}$ & $\begin{array}{l}0 \\
0 \\
5 \\
5\end{array}$ & $\begin{array}{l}\mathscr{W} \\
0 \\
\frac{1}{\supset}\end{array}$ & $\begin{array}{l}\stackrel{\varphi}{\mathscr{E}} \\
\stackrel{5}{5}\end{array}$ & 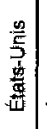 & 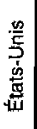 & 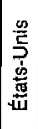 \\
\hline 只 & 查 & $\begin{array}{l}\stackrel{m}{W} \\
\stackrel{W}{\Gamma}\end{array}$ & 总 & 总 & $\begin{array}{l}\text { 萿 } \\
\stackrel{-}{-}\end{array}$ & $\begin{array}{l}\text { 苟 } \\
\text { 号 }\end{array}$ & $\begin{array}{l}\text { 芯 } \\
\text { O }\end{array}$ & 葛 & 吕 & $\begin{array}{l}\stackrel{6}{0} \\
\stackrel{\rho}{\sigma}\end{array}$ & 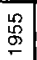 & 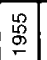 & $\begin{array}{l}0 \\
\stackrel{0}{\circ} \\
\end{array}$ & $\begin{array}{l}\mathscr{8} \\
\stackrel{6}{\sigma} \\
\end{array}$ & 总 & 䓵 & \begin{tabular}{l} 
总 \\
\hdashline
\end{tabular} & 总 & 氮 & 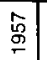 & $\begin{array}{l}\text { 点 } \\
\text { Oे }\end{array}$ & 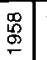 & 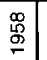 & $\begin{array}{l}\infty \\
\stackrel{\circ}{\circ}\end{array}$ & $\begin{array}{l}\text { \% } \\
\stackrel{5}{\sigma}\end{array}$ \\
\hline
\end{tabular}




\begin{tabular}{|c|c|c|c|c|c|c|c|c|c|c|c|c|c|c|c|c|c|c|c|c|c|}
\hline$\frac{8}{8}$ & & 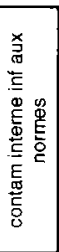 & 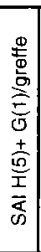 & 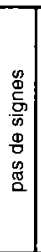 & 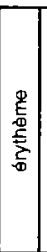 & 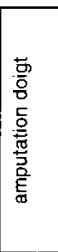 & 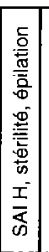 & 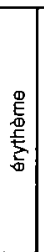 & 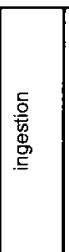 & & & $\frac{0}{\bar{d}}$ & 乔 & & & & & & & : & \\
\hline 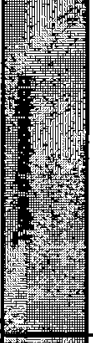 & 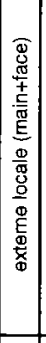 & 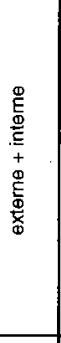 & 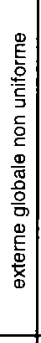 & 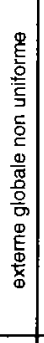 & 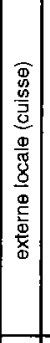 & 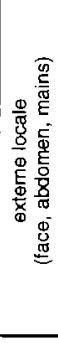 & 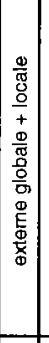 & 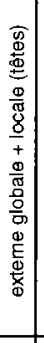 & 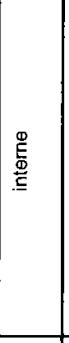 & 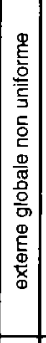 & $\begin{array}{c}\frac{\Phi}{\mathrm{g}} \\
\frac{\mathrm{o}}{\mathrm{g}} \\
\frac{\mathrm{g}}{\mathrm{g}} \\
\frac{\mathrm{g}}{\mathrm{g}}\end{array}$ & 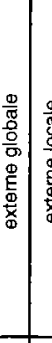 & 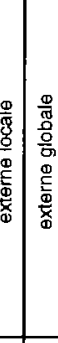 & $\begin{array}{l}\frac{0}{8} \\
\frac{0}{0} \\
\frac{0}{6} \\
\frac{9}{8} \\
\frac{0}{6}\end{array}$ & 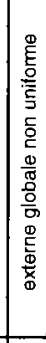 & 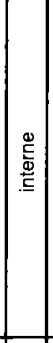 & 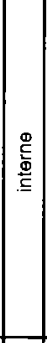 & 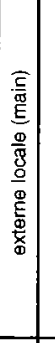 & & 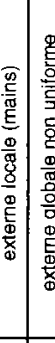 & 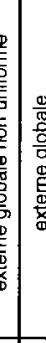 \\
\hline & 0 & 0 & $\underset{\sim}{\stackrel{\vec{~}}{\sim}}$ & $\circ$ & 0 & 0 & 0 & 0 & $\begin{array}{l}\frac{\bar{m}}{0} \\
\frac{\pi}{\pi} \\
\pm \\
-\end{array}$ & 0 & 0 & $\begin{array}{l}\stackrel{0}{0} \\
\stackrel{0}{\circ}\end{array}$ & 00 & 0 & 0 & 0 & 0 & 0 & - & 0 & $\infty$ \\
\hline 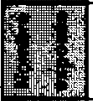 & - & $m$ & $\infty$ & $\mathrm{N}$ & - & - & -1 & $\infty$ & - & $a$ & $\bar{\wedge}$ & - & $-1+$ & - & - & $\sim$ & $\sim$ & $r$ & $\infty$ & $F$ & $=\tilde{8}$ \\
\hline 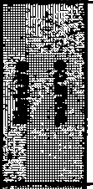 & $\begin{array}{l}0 \\
0 \\
0 \\
0 \\
0 \\
\Phi\end{array}$ & 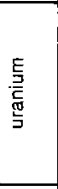 & 葛 & ฉั & 8 & 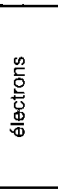 & 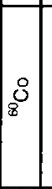 & $\underset{\mathbb{L}}{\rtimes}$ & 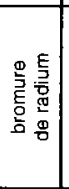 & 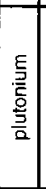 & 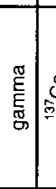 & $\begin{array}{l}8 \\
0 \\
0 \\
0\end{array}$ & 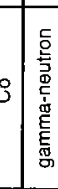 & 佂 & 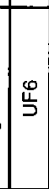 & $\frac{\overrightarrow{2}}{2}$ & $\frac{g^{\circ}}{\frac{0}{N}}$ & x & $m^{I}$ & $\stackrel{x}{x}$ & \\
\hline 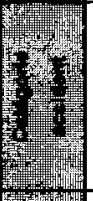 & 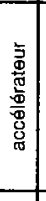 & 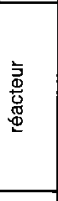 & $\mid \begin{array}{l}\frac{9}{0} \\
\frac{\mathrm{g}}{\bar{t}}\end{array}$ & 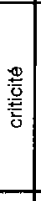 & 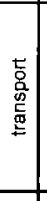 & 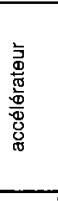 & 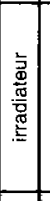 & $\frac{c}{\frac{0}{5}}$ & & 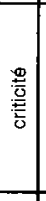 & 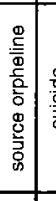 & $\begin{array}{l}\frac{\Phi}{0} \\
: \frac{\overline{0}}{\bar{O}} \\
\overline{\bar{c}}\end{array}$ & 兽 & 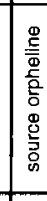 & 罯 & & & 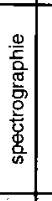 & & |. & 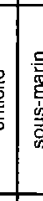 \\
\hline & $x$ & x & $x$ & $\$$ & - & $\Phi$ & ar & - & $\Sigma$ & $\frac{8}{2}$ & -10 & - & - a & $\sim$ & $y$ & $m$ & $r$ & - & $\alpha=$ & $\Sigma x$ & $=\bar{z}$ \\
\hline $\begin{array}{r} \\
x^{2} \\
x^{2}\end{array}$ & 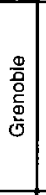 & 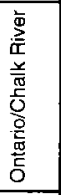 & $\begin{array}{l}\text { a } \\
\stackrel{0}{5} \\
\end{array}$ & 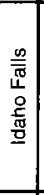 & 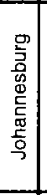 & $\begin{array}{l}\frac{9}{3} \\
\frac{\bar{g}}{\bar{g}} \\
\frac{\vec{g}}{\bar{g}} \\
\frac{0}{\alpha}\end{array}$ & 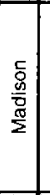 & $\begin{array}{l}5 \\
\overline{0} \\
\frac{0}{0} \\
0 \\
\end{array}$ & & 竧 & 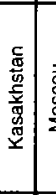 & ఫ. & \begin{tabular}{l|l} 
\\
\\
\end{tabular} & $\begin{array}{l}\vec{z} \\
\text { 品 } \\
\text { 总 }\end{array}$ & 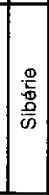 & 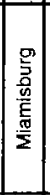 & 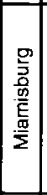 & 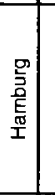 & & 訔 & 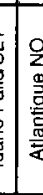 \\
\hline 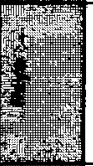 & 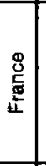 & 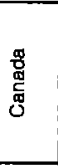 & 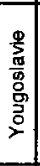 & 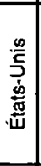 & 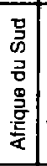 & 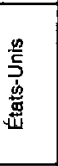 & 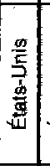 & 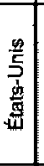 & $\begin{array}{l}\frac{9}{8} \\
\frac{8}{5}\end{array}$ & $\begin{array}{l}n \\
0 \\
\frac{2}{5}\end{array}$ & $\begin{array}{l}0 \\
0 \\
\frac{0}{5}\end{array}$ & $\begin{array}{ll}n \\
0 \\
\frac{p}{5} \\
\frac{5}{5}\end{array}$ & 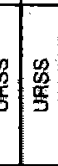 & 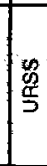 & $\mid \begin{array}{l}\mathscr{P} \\
\stackrel{9}{5} \\
\frac{9}{5}\end{array}$ & 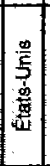 & 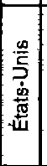 & 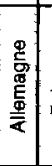 & 总 & 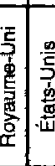 & 票 \\
\hline Fit & \begin{tabular}{|l|l|}
\multirow{2}{\circ}{} \\
\end{tabular} & 岕 & $\begin{array}{l}\stackrel{\infty}{0} \\
\stackrel{\leftrightarrow}{\circ} \\
\end{array}$ & $\underset{\circ}{\stackrel{\circ}{\circ}}$ & 昫 & $\underset{\stackrel{8}{\circ}}{\stackrel{\circ}{\circ}}$ & \begin{tabular}{l|}
$\circ$ \\
$\circ$ \\
\end{tabular} & $\begin{array}{l}\circ \\
\stackrel{\circ}{\circ} \\
\end{array}$ & 怘 & $\underset{\$}{\stackrel{8}{\circ}}$ & $\stackrel{\circ}{\circ}$ & $\stackrel{\circ}{\circ}$ & 㣎 & 产 & ष & \begin{tabular}{|l} 
\\
\end{tabular} & $\stackrel{\Phi}{\circ}$ & 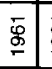 & \begin{tabular}{l|l}
$\overline{\mathscr{g}}$ & $\bar{\delta}$ \\
& $\bar{g}$ \\
\end{tabular} & \begin{tabular}{l|l}
$\overline{8}$ & $\bar{g}$ \\
\end{tabular} & 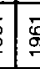 \\
\hline
\end{tabular}




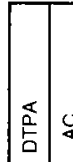

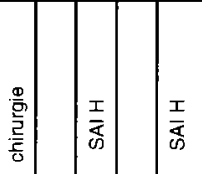

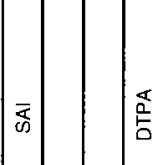

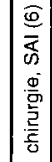

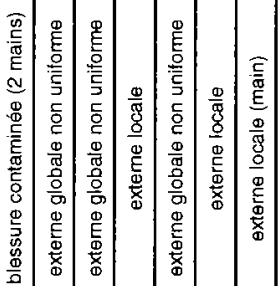

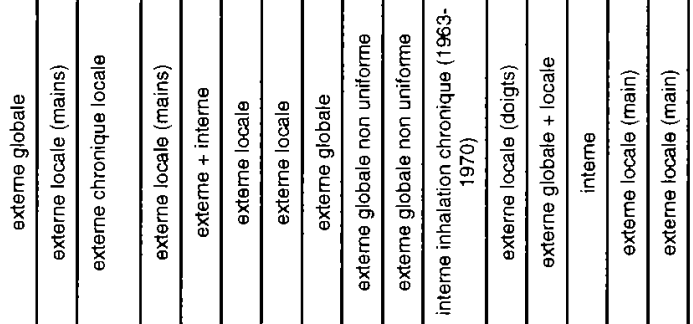

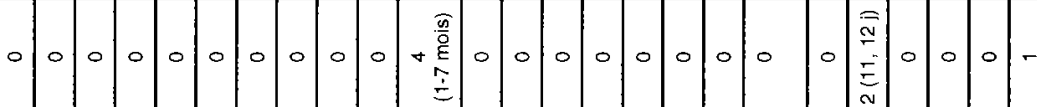

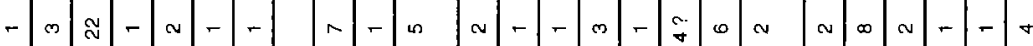

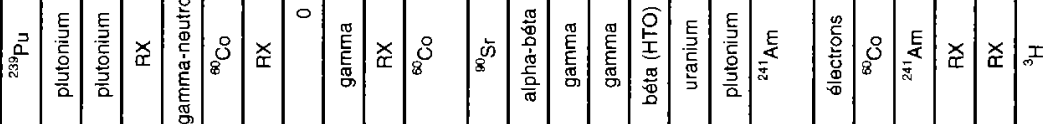

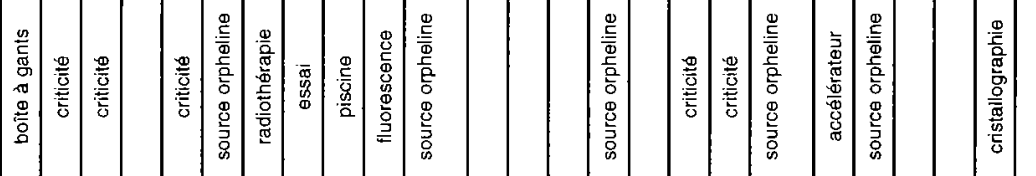

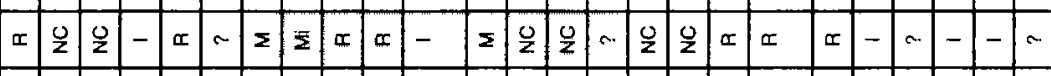

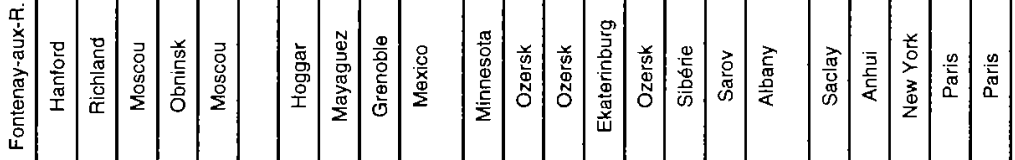

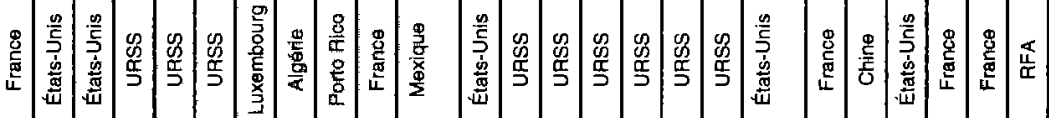

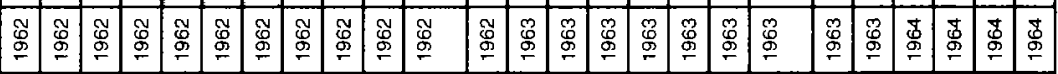




\begin{tabular}{|c|c|c|c|c|c|c|c|c|c|c|c|c|c|c|c|c|c|c|c|c|c|c|c|}
\hline$\frac{3}{8}$ & & & 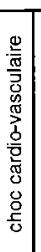 & 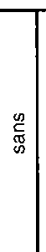 & 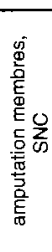 & $\begin{array}{l}\widehat{E} \\
\frac{0}{\bar{g}} \\
\frac{2}{\overline{\frac{\pi}{0}}}\end{array}$ & 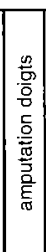 & & 爱 & $\mid$\begin{tabular}{c|}
$\frac{9}{5}$ \\
$\overline{2}$ \\
$\frac{2}{\overline{2}}$ \\
0
\end{tabular} & & & & 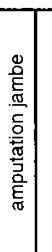 & & & & & $\begin{array}{l}\frac{0}{5} \\
\frac{5}{5} \\
\frac{2}{2} \\
\frac{0}{0}\end{array}$ & & & & \\
\hline 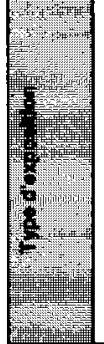 & 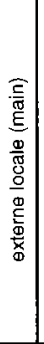 & 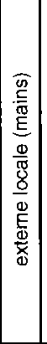 & 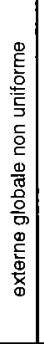 & 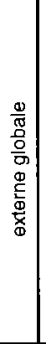 & 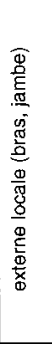 & 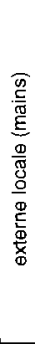 & 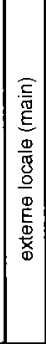 & 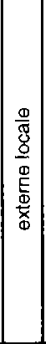 & 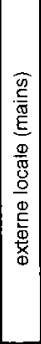 & 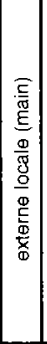 & 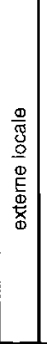 & 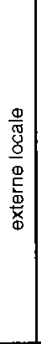 & 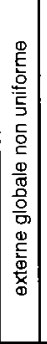 & 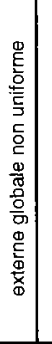 & & 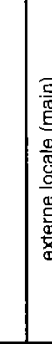 & & 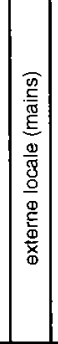 & 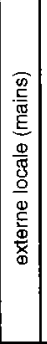 & 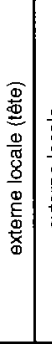 & 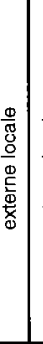 & & 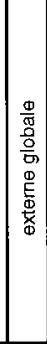 \\
\hline ISt & 0 & 0 & $\begin{array}{l}\text { E } \\
\stackrel{g}{9} \\
-\end{array}$ & 0 & 0 & 0 & 0 & 0 & 0 & 0 & 0 & 0 & 0 & 0 & 0.0 & 0.0 & - & 0 & 0 & 0 & 0 & & 0 \\
\hline 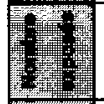 & - & N & $\infty$ & - & - & $m$ & $1-$ & - & - & r & - & $r$ & - & ल & t & - & - & - & - & - & 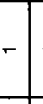 & - & is \\
\hline 12 & $\frac{2}{x}$ & $\therefore$ & 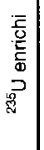 & & 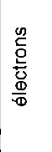 & শx & 쭘 & $\underset{\mathbb{x}}{x}$ & 줌 & 줌 & xx & 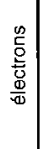 & 造 & $\begin{array}{l}\bar{z} \\
\frac{\bar{y}}{\bar{v}} \\
\bar{\phi} \\
\mathrm{g} \\
\mathrm{g}\end{array}$ & . & $\begin{array}{l}\vec{a} \\
a^{3}\end{array}$ & 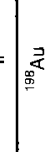 & $\stackrel{x}{x}$ & $\stackrel{x}{\mathbb{x}}$ & $\stackrel{x}{x}$ & हु & & u \\
\hline 8 & & & $\begin{array}{l}\frac{D}{\mathrm{t}} \\
\frac{\bar{t}}{\mathrm{t}}\end{array}$ & 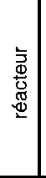 & 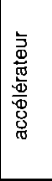 & & 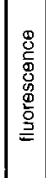 & 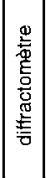 & & & 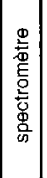 & 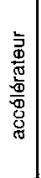 & 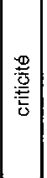 & 兽 & & & & & & & 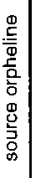 & & 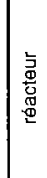 \\
\hline 0 & - & -1 & $\frac{1}{2}$ & 인 & - & $\approx$ & - & $\cdots$ & $\Sigma$ & $\Sigma$ & a. & 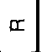 & $\frac{0}{2}$ & 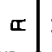 & $\Sigma 0$ & . -1 & $-\mid \Sigma$ & - & $\pi$ & - & $\frac{0}{2}$ & & r \\
\hline (5) & 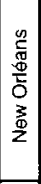 & 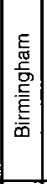 & $\begin{array}{l}\frac{2}{3} \\
\frac{1}{\alpha x} \\
\bar{c} \\
\frac{0}{3} \\
3\end{array}$ & 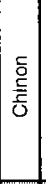 & 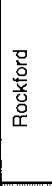 & 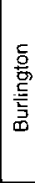 & & & $\begin{array}{l}\stackrel{\Phi}{0} \\
\stackrel{0}{\mathrm{E}} \\
\stackrel{9}{\mathrm{~g}}\end{array}$ & 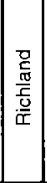 & & $\begin{array}{l}\text { 总 } \\
\text { o. } \\
\text { ¿ }\end{array}$ & 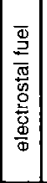 & $\overline{\frac{0}{2}}$ & $\begin{array}{ll} & \\
\mathrm{c} \\
\mathrm{w} \\
\mathrm{t} \\
\mathrm{c} \\
\mathrm{c}\end{array}$ & 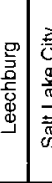 & 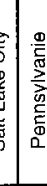 & $\begin{array}{l}-\frac{0}{a x} \\
0\end{array}$ & $\begin{array}{l}\frac{5}{0} \\
\frac{0}{2} \\
\frac{\overrightarrow{0}}{2}\end{array}$ & & 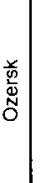 & $\begin{array}{l}\frac{\partial}{0} \\
0 \\
\frac{0}{2} \\
\frac{0}{2}\end{array}$ & \\
\hline 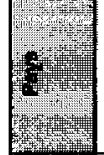 & 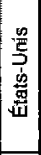 & 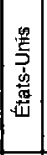 & 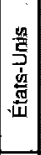 & 孪 & 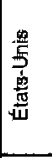 & 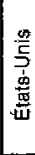 & 產 & 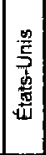 & 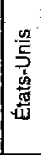 & 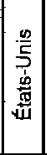 & 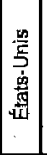 & 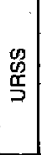 & $\begin{array}{l}0 \\
02 \\
0 \\
2\end{array}$ & 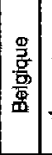 & 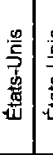 & 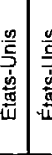 & 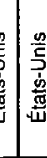 & $\begin{array}{l}8 \\
8 \\
\frac{5}{\mathbb{2}} \\
14\end{array}$ & 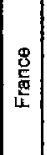 & $\begin{array}{l}\frac{n}{8} \\
\frac{8}{5}\end{array}$ & $\frac{n}{5}$ & & $\begin{array}{l}y \\
0 \\
\underline{a} \\
=\end{array}$ \\
\hline (8) & $\begin{array}{l}\stackrel{\sharp}{\circ} \\
\stackrel{\circ}{\circ}\end{array}$ & \begin{tabular}{|l|} 
\\
怘 \\
\end{tabular} & \begin{tabular}{|l|} 
\\
\\
0 \\
\end{tabular} & \begin{tabular}{|l|}
$\stackrel{0}{\circ}$ \\
$\stackrel{\circ}{\circ}$ \\
\end{tabular} & 兽 & $\stackrel{\circ}{\circ}$ & \begin{tabular}{|l} 
怘 \\
$\stackrel{9}{9}$
\end{tabular} & 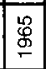 & 岁 & 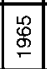 & \begin{tabular}{|l|} 
\\
\\
\end{tabular} & 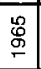 & \begin{tabular}{|l|} 
\\
\\
\end{tabular} & $\mid$ & 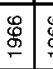 & 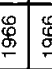 & $\begin{array}{l}0 \\
0 \\
0\end{array}$ & $\begin{array}{l}\mathscr{0} \\
\stackrel{0}{\circ} \\
-\end{array}$ & \begin{tabular}{|l|} 
\\
$\stackrel{\circ}{\circ}$ \\
\end{tabular} & 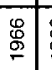 & 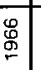 & 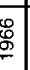 & $\stackrel{\circ}{\circ}$ \\
\hline
\end{tabular}




\begin{tabular}{|c|c|c|c|c|c|c|c|c|c|c|c|c|c|c|c|c|c|c|c|c|c|c|c|c|}
\hline & & 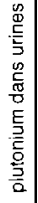 & & & 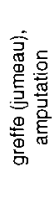 & 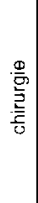 & & & & 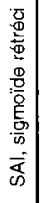 & 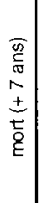 & 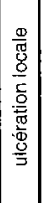 & & $\begin{array}{l}a \\
0 \\
0\end{array}$ & 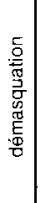 & 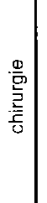 & & & 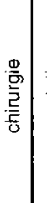 & 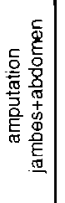 & 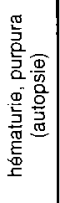 & & 黑 & \\
\hline 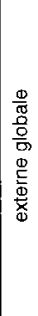 & 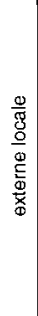 & $\begin{array}{l}\stackrel{\Phi}{5} \\
\stackrel{\Phi}{\leftrightarrows}\end{array}$ & 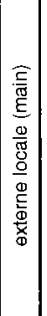 & 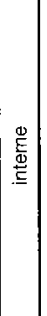 & 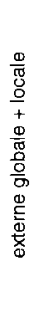 & 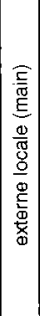 & 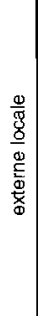 & 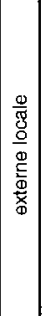 & 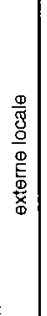 & 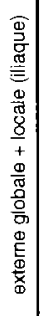 & 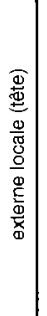 & 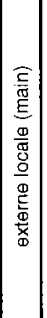 & 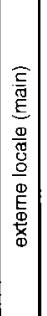 & 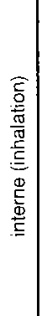 & 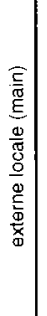 & 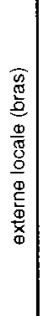 & 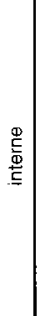 & $\begin{array}{l}\mathbb{D} \\
\stackrel{\mathbb{E}}{\mathbf{D}} \\
. \underline{\underline{E}}\end{array}$ & 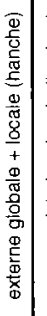 & 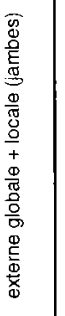 & 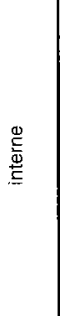 & 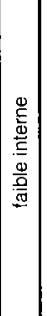 & 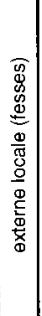 & 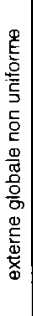 \\
\hline 0 & 0 & 0 & 0 & 0 & 0 & 0 & 0 & 0 & $\circ$ & 0 & 0 & 0 & 0 & 0 & 0 & 0 & 0 & 兽 & 0 & 0 & $\stackrel{\overline{8}}{0}$ & $\circ$ & 0 & $\sim$ \\
\hline$\propto$ & - & - & $\infty$ & - & CD & - & - & - & $\tau$ & - & - & - & - & $\sim$ & $r$ & $r$ & $\infty$ & - & - & - & - & 8 & - & $N$ \\
\hline & ج્પ & 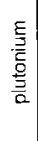 & 늘 & 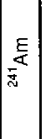 & 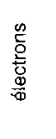 & 8 & 爻 & $\underset{\mathbb{I}}{x}$ & $\begin{array}{c}0 \\
6 \\
\%\end{array}$ & 8 & 爻 & 象 & $\underset{w}{\underline{w}}$ & $\frac{E}{\alpha}$ & 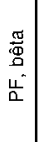 & $\underset{\mathbb{x}}{x}$ & 胥 & 疋 & 능 & $\begin{array}{l}83 \\
0 \\
0\end{array}$ & 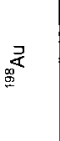 & $\begin{array}{l}\underline{\underline{\underline{I}}} \\
\text { 妾 } \\
\text { 흠 }\end{array}$ & $\frac{\text { 늘 }}{\Phi, ~}$ & 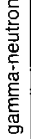 \\
\hline 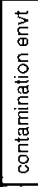 & 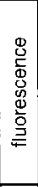 & 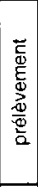 & 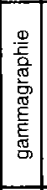 & & 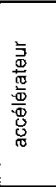 & 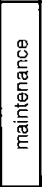 & & & & & 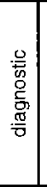 & 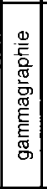 & & & 离 & 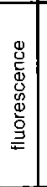 & & & 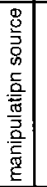 & 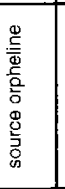 & $\frac{\bar{\Xi}}{\bar{\Phi}}$ & 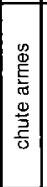 & 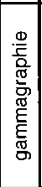 & $\begin{array}{l}\text { 吾 } \\
\frac{\mathrm{O}}{\mathrm{E}} \\
\mathrm{E}\end{array}$ \\
\hline a. & $\llbracket$ & $\mathbf{O}$ & - & $\propto$ & $\Sigma$ & $\Sigma$ & - & - & - & $\cdots$ & $\Sigma$ & - & - & $\llbracket$ & 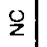 & 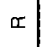 & $\approx$ & $\Sigma$ & - & - & $\Sigma$ & $\bar{\Sigma}$ & - & 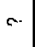 \\
\hline & & $\frac{\frac{a}{\pi}}{5}$ & & $\begin{array}{l}\text { 민 } \\
\text { 产 } \\
\text { 응 } \\
\frac{8}{0} \\
0\end{array}$ & 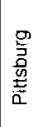 & 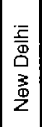 & $\begin{array}{l}\stackrel{D}{N} \\
\stackrel{2}{\vec{Z}} \\
\stackrel{2}{*}\end{array}$ & 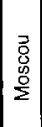 & $\mid \begin{array}{l}z \\
\bar{o} \\
0 \\
\Sigma \\
\Sigma\end{array}$ & & $\begin{array}{l}\overrightarrow{0} \\
\stackrel{u}{\circ} \\
\stackrel{o}{\Sigma} \\
\stackrel{0}{\Sigma}\end{array}$ & & 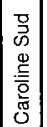 & & 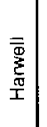 & & 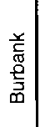 & $\begin{array}{l}\frac{.}{0} \\
\frac{9}{5} \\
\frac{0}{3} \\
\frac{.0}{3}\end{array}$ & & $\begin{array}{l}\frac{9}{\pi} \\
\frac{\pi}{0} \\
\pi \\
\pi\end{array}$ & 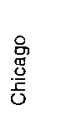 & $\frac{\mathbf{m}}{\vec{E}}$ & & 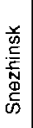 \\
\hline$\frac{\mathscr{E}}{\frac{E}{E}}$ & 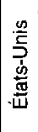 & 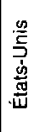 & 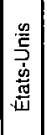 & 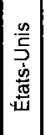 & 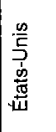 & 畺 & 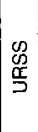 & $\begin{array}{l}\text { か } \\
\text { 䓂 }\end{array}$ & $\begin{array}{l}0 \\
0 \\
0 \\
\frac{I}{2}\end{array}$ & $\begin{array}{l}\text { 另 } \\
\text { 息 }\end{array}$ & $\begin{array}{l}0 \\
0 \\
\frac{5}{5}\end{array}$ & 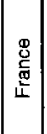 & 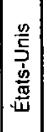 & 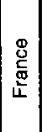 & 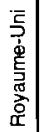 & 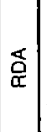 & 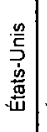 & 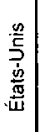 & $\underset{\mathbb{u}}{\stackrel{\leftarrow}{\mathbb{x}}}$ & 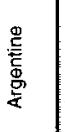 & 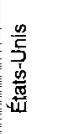 & 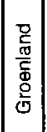 & 虹 & $\begin{array}{l}\frac{\mathscr{L}}{\mathscr{L}} \\
\frac{\mathrm{L}}{5}\end{array}$ \\
\hline $\begin{array}{l}\mathscr{8} \\
\stackrel{\circ}{\circ}\end{array}$ & 参 & $\stackrel{\circ}{\circ}$ & 兽 & 灾 & 兽 & 鸟 & $\begin{array}{l}1 \\
\stackrel{\circ}{\circ}\end{array}$ & $\begin{array}{l}\text { 总 } \\
\stackrel{2}{2}\end{array}$ & $\begin{array}{l}0 \\
0 \\
0\end{array}$ & $\stackrel{\grave{\circ}}{\circ}$ & $\stackrel{\widehat{Q}}{\circ}$ & 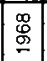 & $\begin{array}{l}\infty \\
\$ \\
\$\end{array}$ & 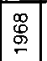 & $\begin{array}{l}\infty \\
\stackrel{8}{\circ} \\
\stackrel{6}{-}\end{array}$ & $\begin{array}{l}\infty \\
\stackrel{\infty}{\infty} \\
\stackrel{\infty}{-}\end{array}$ & 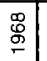 & $\begin{array}{l}\$ \\
\Phi\end{array}$ & 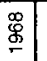 & $\begin{array}{l}\mathscr{D} \\
\stackrel{8}{\%} \\
=\end{array}$ & $\begin{array}{l}\mathscr{8} \\
\underset{\sigma}{\sigma}\end{array}$ & 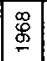 & 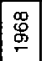 & $\begin{array}{l}\stackrel{8}{8} \\
\stackrel{g}{\sigma} \\
\end{array}$ \\
\hline
\end{tabular}




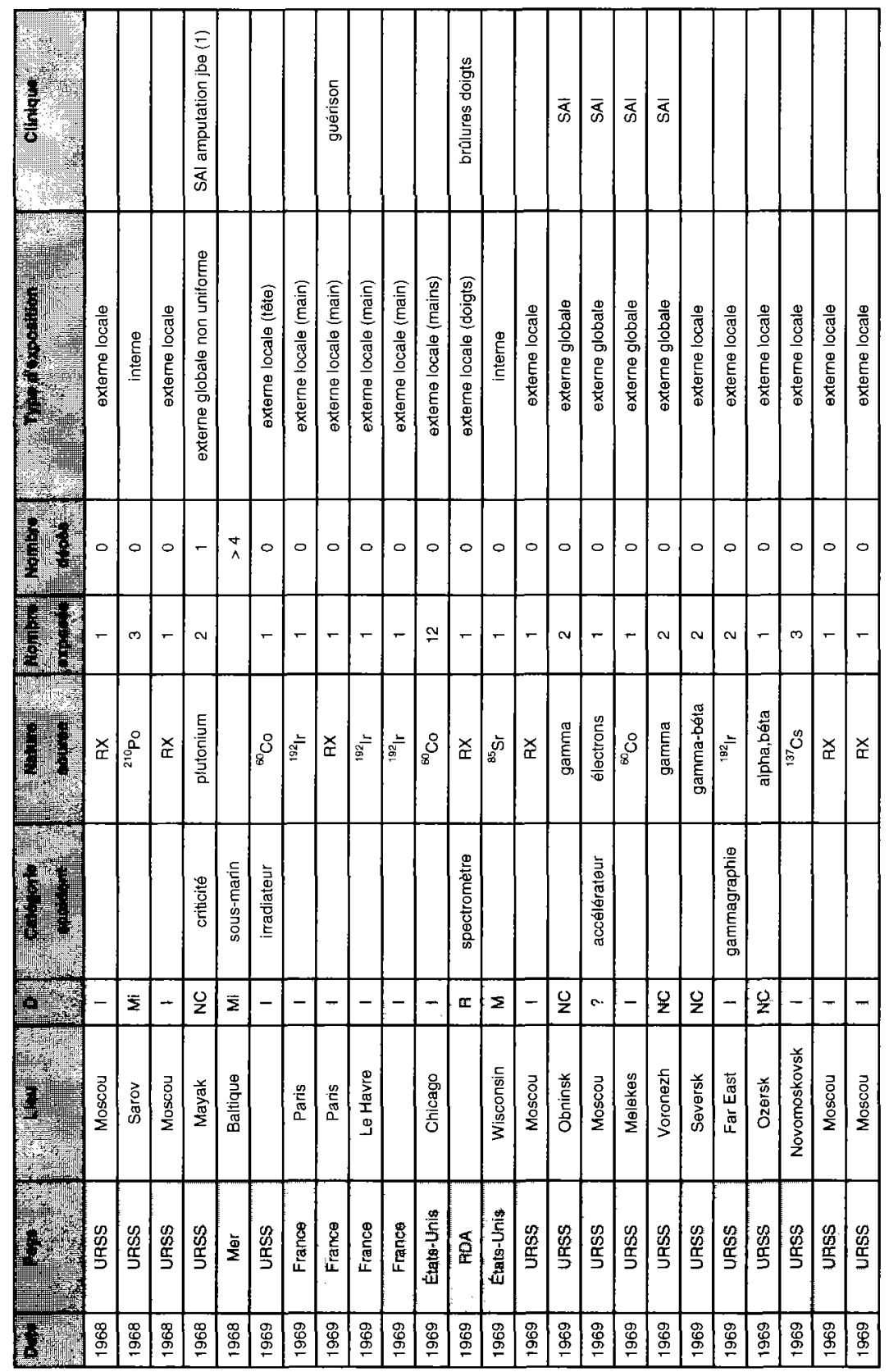




\begin{tabular}{|c|c|c|c|c|c|c|c|c|c|c|c|c|c|c|c|c|c|c|c|c|c|c|c|}
\hline & & 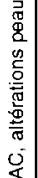 & & & & & 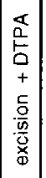 & 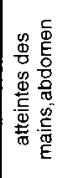 & & & & & $\begin{array}{l}\bar{a} \\
b\end{array}$ & & & $\begin{array}{l}\bar{a} \\
\infty \\
\infty\end{array}$ & & 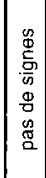 & & & & 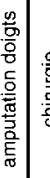 & \\
\hline 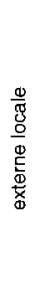 & 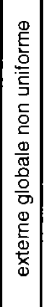 & 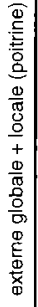 & 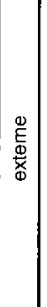 & 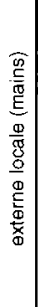 & 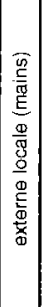 & 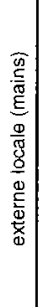 & 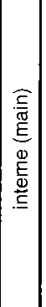 & 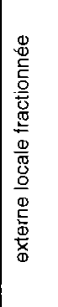 & 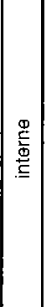 & 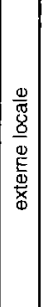 & & 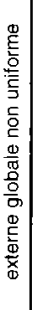 & 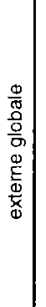 & 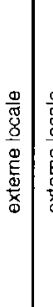 & 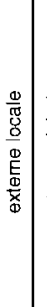 & 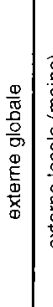 & 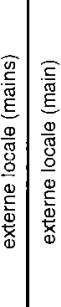 & 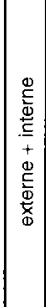 & 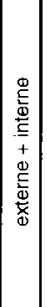 & 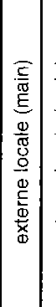 & 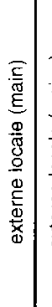 & 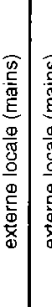 & 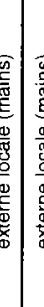 \\
\hline 0 & 0 & 0 & 0 & 0 & 0 & 0 & $\circ$ & 0 & $\circ$ & 0 & $\circ$ & 0 & $\circ$ & $\circ 0$ & 0 & $\infty$ & \begin{tabular}{l|l}
$\circ$ & 0
\end{tabular} & 0 & 0 & 0 & $\circ$ & $\circ$ & $\circ$ \\
\hline- & t & - & $\cdots$ & - & - & -1 & - & $m$ & - & - & - & $\mathrm{v}$ & - & - & - & \begin{tabular}{l|l}
$\wedge$ & 0
\end{tabular} & $\sim-$ & - & $\infty$ & - & - & - & - \\
\hline$\underset{\mathbb{T}}{\underset{x}{x}}$ & $\frac{u}{a}$ & $=$ & $\begin{array}{l}8 \\
8\end{array}$ & $\underset{x}{x}$ & $\mid \underset{x}{x}$ & $\cdots$ & 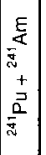 & 짐 & a & $\underset{\mathbb{E}}{x}$ & 品 & 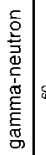 & 8 & 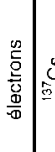 & है & 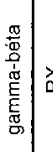 & x $\underset{x}{x}$ & $\begin{array}{l}E \\
\tilde{J} \\
\tilde{J}\end{array}$ & 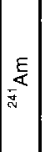 & $\underset{x}{x}$ & $\underset{\mathbb{x}}{x}$ & 8 & $=$ \\
\hline & 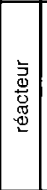 & 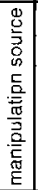 & 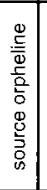 & & & 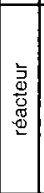 & & 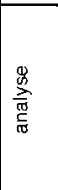 & & 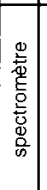 & & 总 & & 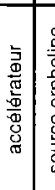 & 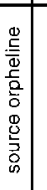 & & 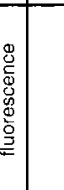 & 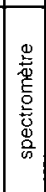 & & & & & \\
\hline- & $\simeq$ & - & $\sim$ & $x$ & $\simeq$ & $\approx$ & $\frac{0}{z}$ & 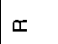 & $\Sigma$ & $\sim$ & $\sim$ & $\llbracket$ & - & $\therefore$ & $\therefore$. & $\frac{v}{z}$ & $-\infty$ & - & - & $\sim$ & r. & - & - \\
\hline 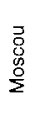 & & 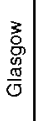 & 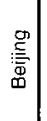 & 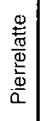 & \begin{tabular}{|l|}
$\frac{0}{0}$ \\
$\frac{0}{0}$ \\
$\frac{0}{10}$ \\
$\frac{0}{0}$
\end{tabular} & 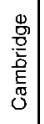 & 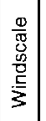 & 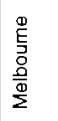 & 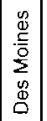 & & 趉 & $\frac{\vec{a}}{\underline{x}}$ & & 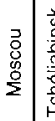 & 总 & 总 & 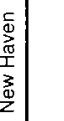 & & & & & 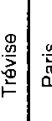 & 吠 \\
\hline $\begin{array}{l}\text { 足 } \\
\text { 苟 }\end{array}$ & $\begin{array}{l}n \\
\tilde{n} \\
\frac{\tilde{m}}{5}\end{array}$ & 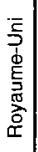 & 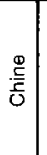 & 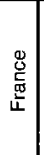 & 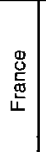 & 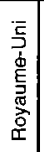 & 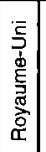 & 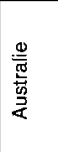 & 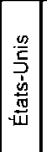 & 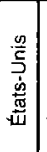 & 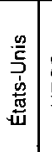 & $\begin{array}{l}\text { 怘 } \\
\text { 芹 } \\
\frac{5}{5}\end{array}$ & $\begin{array}{l}0 \\
0 \\
0 \\
\frac{1}{J} \\
\end{array}$ & $\begin{array}{l}0 \\
0 \\
\frac{2}{5} \\
\end{array}$ & $\begin{array}{l}\mathscr{D} \\
\mathbb{2} \\
\stackrel{\mathbb{I}}{丂}\end{array}$ & 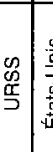 & 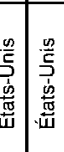 & $\frac{\mathbb{S}}{\mathbb{T}}$ & 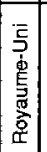 & 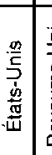 & 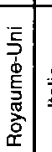 & 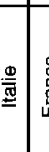 & $\begin{array}{l}0 \\
\frac{0}{0} \\
\frac{1}{0} \\
\end{array}$ \\
\hline$\underset{\stackrel{g}{\circ}}{\stackrel{g}{\sigma}}$ & 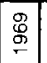 & $\stackrel{\circ}{\circ}$ & $\begin{array}{l}\stackrel{8}{\circ} \\
\stackrel{\circ}{-}\end{array}$ & 呑 & 呑 & 尽 & \begin{tabular}{|l|} 
\\
$\vdots$ \\
0
\end{tabular} & 용 & \begin{tabular}{|l|} 
\\
号 \\
\end{tabular} & \begin{tabular}{|l|} 
\\
\\
\end{tabular} & 只 & $\begin{array}{l}\stackrel{0}{0} \\
0\end{array}$ & $\begin{array}{l}\stackrel{?}{0} \\
\stackrel{0}{o}\end{array}$ & 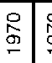 & $\begin{array}{l}\text { 只 } \\
\\
\end{array}$ & $\begin{array}{ll}2 \\
0 \\
0\end{array}$ & 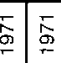 & $\underset{\stackrel{\sigma}{\sigma}}{\stackrel{\sigma}{\sigma}}$ & 帛 & $\underset{\sigma}{\sigma}$ & 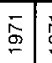 & $\bar{\sigma}$ & 5 \\
\hline
\end{tabular}




\begin{tabular}{|c|c|c|c|c|c|c|c|c|c|c|c|c|c|c|c|c|c|c|c|c|c|c|c|c|}
\hline$\left[\begin{array}{l}8 \\
0 \\
0\end{array}\right.$ & & 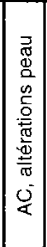 & & & & $\frac{I}{\bar{\alpha}}$ & & & & & & & 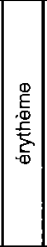 & 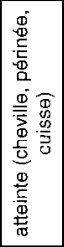 & & & 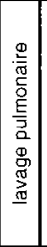 & 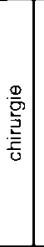 & & & & & & \\
\hline $\begin{array}{l}\mid \\
\frac{5}{8} \\
8 \\
8 \\
\frac{8}{8} \\
\end{array}$ & 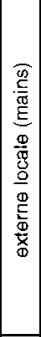 & 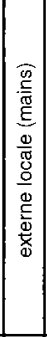 & 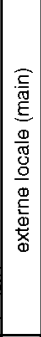 & 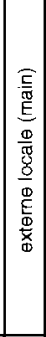 & 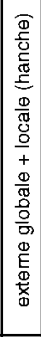 & 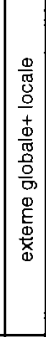 & 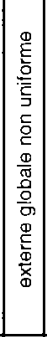 & 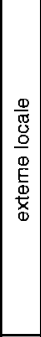 & 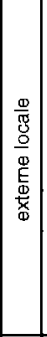 & 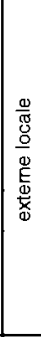 & 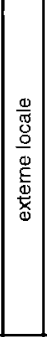 & 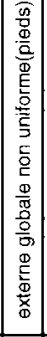 & 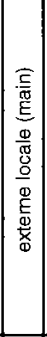 & 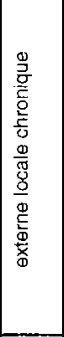 & 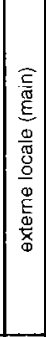 & 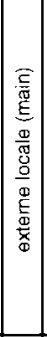 & 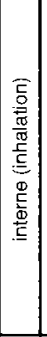 & 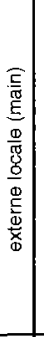 & 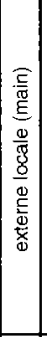 & 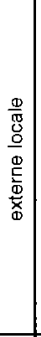 & $\begin{array}{l}\frac{0}{0} \\
\frac{0}{0} \\
\frac{0}{0} \\
\frac{0}{5} \\
\frac{\mathrm{g}}{0}\end{array}$ & 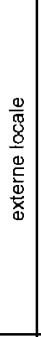 & 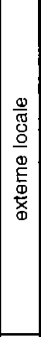 & \\
\hline Whe & 0 & 0 & 0 & 0 & 0 & 0 & $\sim$ & 0 & 0 & 0 & 0 & 0 & 0 & 0 & 0 & 0 & 0 & $\circ$ & 0 & 0 & 0 & 0 & $\circ$ & 0 \\
\hline 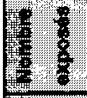 & $N$ & - & - & $1-$ & $\bullet$ & - & $r$ & - & $\begin{array}{l}8 \\
1 \\
1\end{array}$ & - & - & + & - & - & - & - & - & - & - & - & - & $r$ & - & $\bar{\Lambda}$ \\
\hline 8 & 쫌 & 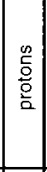 & 8 & $=$ & $=$ & 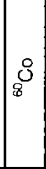 & 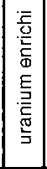 & 8 & \begin{tabular}{|l|}
0 \\
0 \\
0 \\
0
\end{tabular} & $\begin{array}{l}0 \\
0 \\
0\end{array}$ & 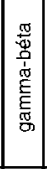 & 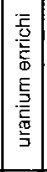 & 짐 & 0 & $\underset{\widetilde{x}}{x}$ & 㐅⿸ & $\begin{array}{l}0 \\
3 \\
0\end{array}$ & 8 & $\underset{x}{x}$ & $\underset{\mathbb{x}}{\underset{x}{x}}$ & 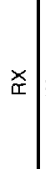 & $\stackrel{\text { }}{\stackrel{*}{\sigma}}$ & $\underset{\mathfrak{a}}{\mathrm{a}}$ & $\underset{\widetilde{\Upsilon}}{\times}$ \\
\hline$\frac{9}{8} \frac{6}{8}$ & & 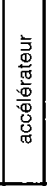 & & & 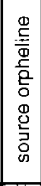 & 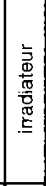 & 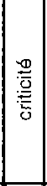 & 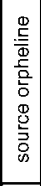 & 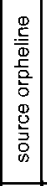 & 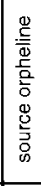 & & $\begin{array}{l}\stackrel{\Phi}{\underline{T}} \\
\stackrel{\bar{t}}{5}\end{array}$ & 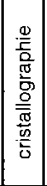 & 胥 & & & & & 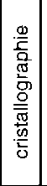 & & & 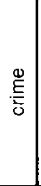 & & \\
\hline & $\pi$ & - & - & - & - & $\pi$ & $\propto$ & $\infty$ & $\cdots$ & $\sim$ & $\cdots$ & $\approx$ & $\approx$ & - & $\sim$ & - & $\infty$ & $\simeq$ & $\simeq$ & - & - & - & - & - \\
\hline 3 & 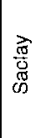 & & 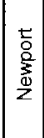 & 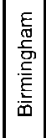 & 营 & 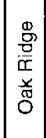 & \begin{tabular}{|l|}
$\frac{3}{\pi}$ \\
$\frac{\pi}{0}$ \\
$\frac{0}{5}$ \\
$\frac{5}{2}$ \\
\end{tabular} & $\frac{\frac{\pi}{2}}{5}$ & 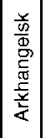 & $\frac{\pi}{5}$ & 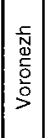 & 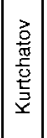 & 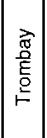 & 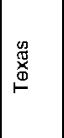 & & $\begin{array}{c}\frac{0}{5} \\
\text { 要 }\end{array}$ & & $\begin{array}{l}\overrightarrow{3} \\
\frac{\pi}{0} \\
\overline{0} \\
\omega\end{array} \mid$ & 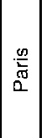 & $\begin{array}{l}\vec{z} \\
0 \\
\frac{0}{2}\end{array}$ & $\begin{array}{l}\vec{z} \\
\dot{o} \\
\frac{0}{2} \\
\end{array}$ & 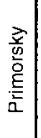 & $\begin{array}{l}\overrightarrow{0} \\
\text { o } \\
\frac{0}{2}\end{array}$ & 蔏 \\
\hline 8 & 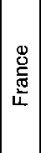 & 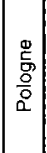 & 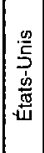 & 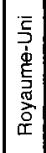 & 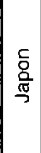 & 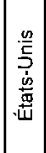 & $\begin{array}{l}0 \\
0 \\
\frac{0}{5} \\
\end{array}$ & $\mid \begin{array}{l}0 \\
0 \\
0 \\
\end{array}$ & $\mid \begin{array}{l}\mathscr{0} \\
\frac{0}{5} \\
\frac{1}{5}\end{array}$ & \begin{tabular}{|l}
0 \\
0 \\
0 \\
$\frac{n}{5}$
\end{tabular} & $\begin{array}{l}\text { 另 } \\
\text { 站 }\end{array}$ & $\mid \begin{array}{l}\mathscr{0} \\
\frac{6}{5}\end{array}$ & 혿 & 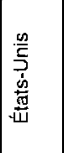 & 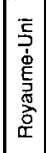 & 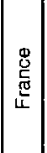 & 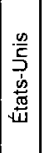 & 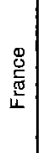 & 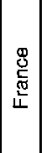 & 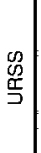 & $\begin{array}{l}\infty \\
0 \\
\tilde{\alpha} \\
\square\end{array}$ & $\begin{array}{l}2 \\
\text { 垈 } \\
\frac{1}{5}\end{array}$ & $\begin{array}{l}n \\
0 \\
0 \\
0 \\
5\end{array}$ & $\begin{array}{l}\text { 总 } \\
\text { 号 }\end{array}$ \\
\hline$\frac{E}{6}$ & 家 & 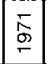 & \begin{tabular}{|l|} 
\\
$\sigma$ \\
\end{tabular} & \begin{tabular}{|l|}
$\bar{\sigma}$ \\
\\
\end{tabular} & 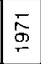 & 市 & \begin{tabular}{|l|}
$\overline{\mathbf{s}}$ \\
$\overline{\mathbf{6}}$ \\
\end{tabular} & 市 & \begin{tabular}{|l|}
$\overline{\hat{\sigma}}$ \\
$\bar{\sigma}$ \\
\end{tabular} & $\frac{\bar{\sigma}}{\sigma}$ & 膍 & $\begin{array}{l}\bar{\sigma} \\
\end{array}$ & \begin{tabular}{|l|}
\multirow{2}{*}{} \\
$\sigma$ \\
$\sigma$ \\
\end{tabular} & 学 & 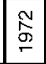 & 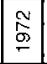 & $\frac{N}{0}$ & $\begin{array}{l}\frac{N}{\alpha} \\
\hat{\sigma} \\
\end{array}$ & 崩 & 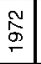 & $\begin{array}{l}\stackrel{N}{N} \\
\stackrel{N}{\sigma} \\
\end{array}$ & 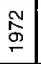 & $\begin{array}{l}\frac{N}{\alpha} \\
\frac{\alpha}{\sigma}\end{array}$ & \\
\hline
\end{tabular}




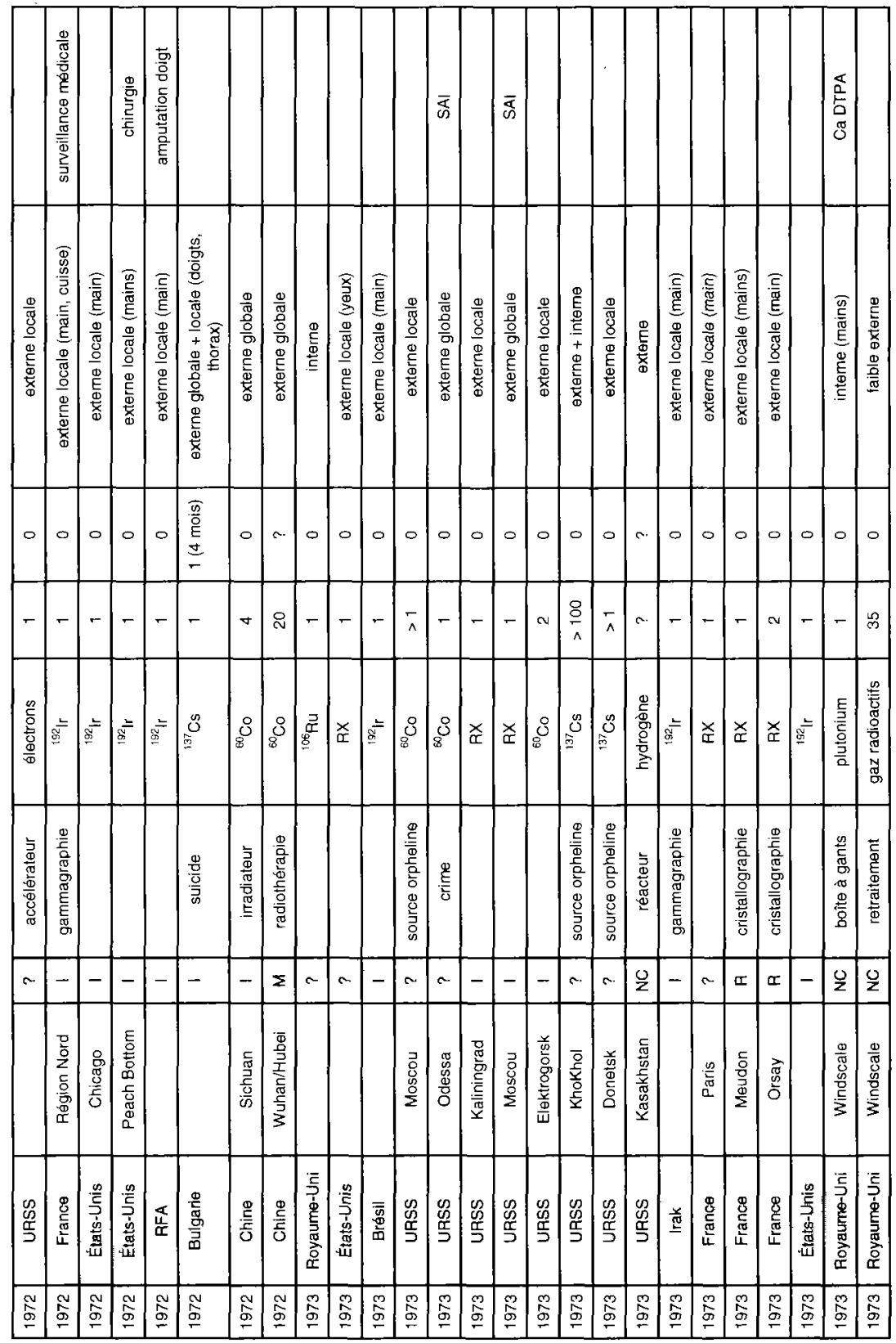




\begin{tabular}{|c|c|c|c|c|c|c|c|c|c|c|c|c|c|c|c|c|c|c|c|c|c|c|c|}
\hline$\frac{2}{d}$ & 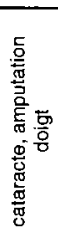 & $\begin{array}{l}\text { 爱 } \\
\text { 产 } \\
\frac{1}{0}\end{array}$ & & 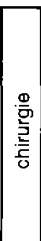 & 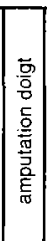 & & 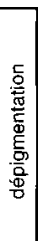 & & 㦞 & 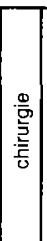 & $\frac{x}{\bar{\alpha}}$ & & & & & $\bar{\delta}$ & & & & \begin{tabular}{|l}
$\frac{0}{6}$ \\
$\frac{6}{3}$
\end{tabular} & & 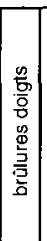 & 空 \\
\hline (1) & 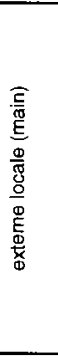 & 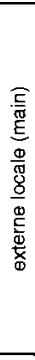 & 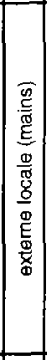 & 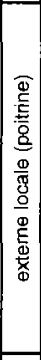 & 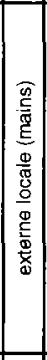 & 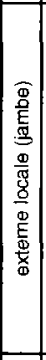 & 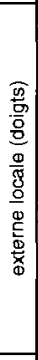 & 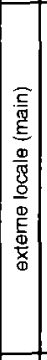 & 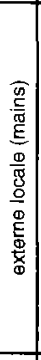 & 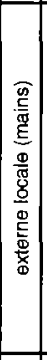 & 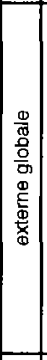 & & 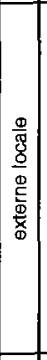 & 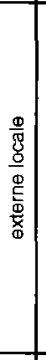 & 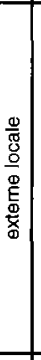 & 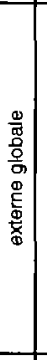 & 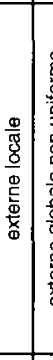 & 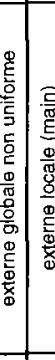 & 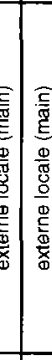 & 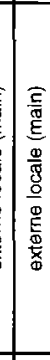 & 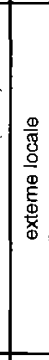 & 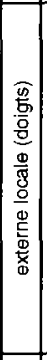 & \\
\hline$y^{4}$ & 0 & 0 & $\circ$ & 0 & 0 & 0 & 0 & 0 & $\circ$ & 0 & 0 & 0 & 0 & 0 & $\circ$ & 0 & 0 & $\begin{array}{l}\stackrel{\vec{\sim}}{=} \\
\stackrel{0}{-}\end{array}$ & 0 & 0 & 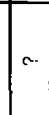 & 0 & 0 \\
\hline 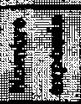 & $\sim$ & $\Gamma$ & $\sim$ & - & $\infty$ & - & - & - & -1 & $\infty$ & - & - & - & - & - & $\sim$ & - & - & - & - & 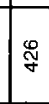 & $\sim$ & $r$ \\
\hline & 8 & 爻 & 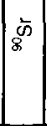 & xx & 잠 & $\stackrel{\underline{*}}{\underline{\underline{\omega}}}$ & $\stackrel{\gtrless}{x}$ & $\stackrel{x}{x}$ & $\Phi^{2}$ & $\stackrel{x}{x}$ & $\mid \begin{array}{l}0 \\
8\end{array}$ & $\stackrel{=}{\underline{m}}$ & 爻 & 童 & 8 & $\begin{array}{l}n \\
0 \\
0 \\
0\end{array}$ & x. & \begin{tabular}{l|l} 
& $\times$ \\
\end{tabular} & $\underline{\underline{c}}$ & 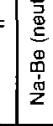 & 8 & : & 童 \\
\hline$\frac{9}{3}+4$ & 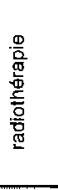 & & & & 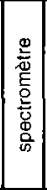 & & 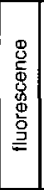 & 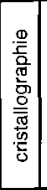 & & 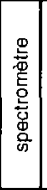 & 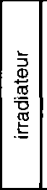 & & & 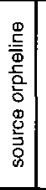 & 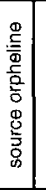 & & 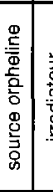 & 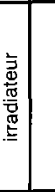 & 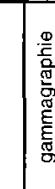 & 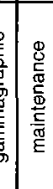 & & 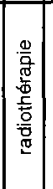 & \\
\hline 8 & $\Sigma$ & $\cdots$ & $\infty$ & $\cdots$ & - & - & $\Sigma$ & $\cdots$ & - & $\cdots$ & - & -1 & -1 & $\infty$ & $\sim$ & $\sim$ & $\therefore-$ & $-a$ & - & 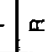 & - & $\mathbf{z}$ & - \\
\hline 145 & & 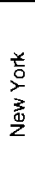 & 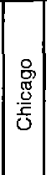 & 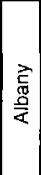 & 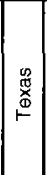 & 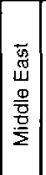 & & & & $\begin{array}{l}\frac{\pi}{5} \\
\frac{5}{2} \\
\frac{2}{2}\end{array}$ & 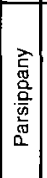 & & 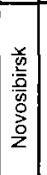 & 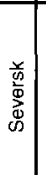 & $\underset{\Phi}{\mathrm{E}}$ & $\begin{array}{l}\frac{x}{0} \\
\frac{0}{0} \\
\frac{0}{3}\end{array}$ & 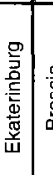 & 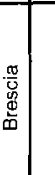 & $\begin{array}{l}\frac{0}{\overline{5}} \\
\frac{.00}{\overline{3}} \\
\end{array}$ & & 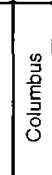 & 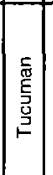 & \\
\hline$\frac{x^{2}}{4}$ & 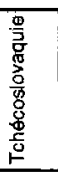 & 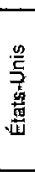 & 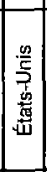 & 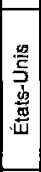 & 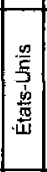 & 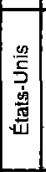 & 윰 & $\stackrel{\text { g }}{\underline{E}}$ & 悹 & 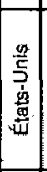 & 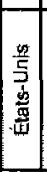 & 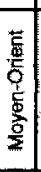 & 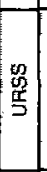 & $\begin{array}{l}0 \\
0 \\
5 \\
5\end{array}$ & 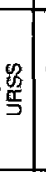 & 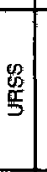 & 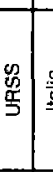 & $\underline{\underline{\underline{Q}}}$ & 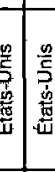 & 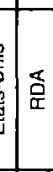 & 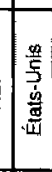 & 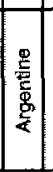 & 离 \\
\hline 8 & 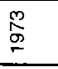 & $\stackrel{5}{\sigma}$ & 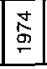 & $\begin{array}{l}\hat{\sigma} \\
\dot{T}\end{array}$ & 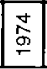 & \begin{tabular}{|l|} 
\\
$\vdots$ \\
$\sigma$
\end{tabular} & \begin{tabular}{c}
\multirow{2}{\alpha}{} \\
$\stackrel{\sigma}{\sigma}$
\end{tabular} & 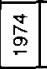 & $\begin{array}{c}\stackrel{ \pm}{\Delta} \\
\stackrel{2}{\sigma} \\
\end{array}$ & 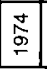 & \begin{tabular}{|l|} 
\\
$\sigma$ \\
$\sigma$
\end{tabular} & $\stackrel{\Phi}{\sigma}$ & $\begin{array}{l}\stackrel{T}{\sigma} \\
\stackrel{\sigma}{\sigma}\end{array}$ & \begin{tabular}{c|}
\multicolumn{1}{|c}{} \\
0 \\
\end{tabular} & $\begin{array}{c}5 \\
\vdots \\
\vdots \\
\end{array}$ & 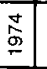 & \begin{tabular}{c|c}
5 \\
$\vdots$ \\
0
\end{tabular} & 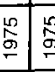 & \begin{tabular}{l|l}
$\sigma$ \\
$\hat{\sigma}$ \\
$\tau$
\end{tabular} & 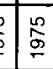 & 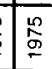 & \begin{tabular}{|l}
$\stackrel{2}{0}$ \\
$\stackrel{0}{6}$ \\
\end{tabular} & \\
\hline
\end{tabular}




\begin{tabular}{|c|c|c|c|c|c|c|c|c|c|c|c|c|c|c|c|c|c|c|c|c|c|c|c|c|}
\hline & & $\bar{\sigma}$ & 㐫 & & 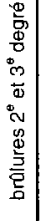 & & & 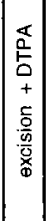 & & 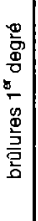 & & & & & 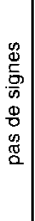 & & 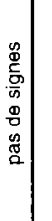 & & & & & & 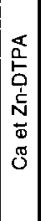 & 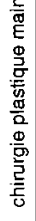 \\
\hline 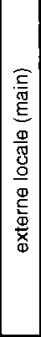 & 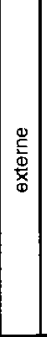 & $\begin{array}{l}\frac{\Phi}{0} \\
\frac{0}{0} \\
\frac{0}{0} \\
\Phi \\
\frac{\Phi}{\mathbf{0}} \\
\frac{\mathbf{5}}{\mathbf{0}}\end{array}$ & 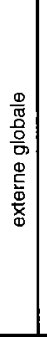 & 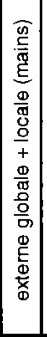 & 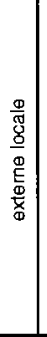 & 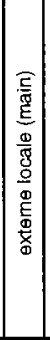 & $\begin{array}{l}\frac{9}{0} \\
\frac{0}{0} \\
\frac{0}{5} \\
0 \\
\frac{E}{0} \\
\frac{0}{0} \\
\mathbb{0}\end{array}$ & 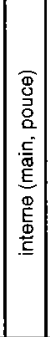 & 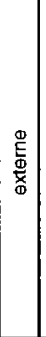 & 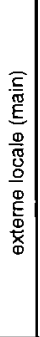 & 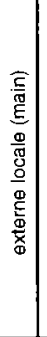 & 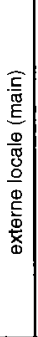 & 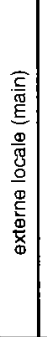 & 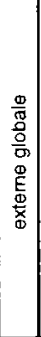 & 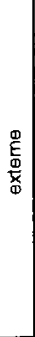 & 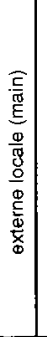 & 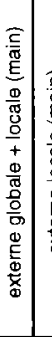 & 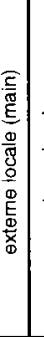 & & & 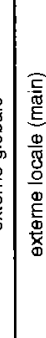 & 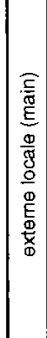 & 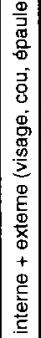 & 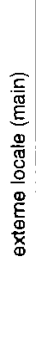 \\
\hline 0 & 0 & 0 & $\tau$ & 0 & 0 & 0 & 0 & 0 & 0 & 0 & 0 & $\circ$ & 0 & 0 & 0 & 0 & $\circ$ & 0 & 0 & 0 & 0 & 0 & 0 & 0 \\
\hline- & $\sim$ & $\sim$ & $m$ & $\approx$ & - & - & - & - & - & - & - & - & - & - & - & - & $\infty$ & - & - & - & - & - & - & - \\
\hline$\stackrel{2}{2}$ & $\stackrel{0}{0}$ & 8 & $\begin{array}{l}8 \\
8\end{array}$ & $=$ & c. & $\underset{\llbracket}{x}$ & $\underset{x}{\times}$ & 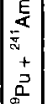 & × & $\underset{\widetilde{x}}{\stackrel{x}{n}}$ & $\frac{\pi}{\pi}$ & $\underset{x}{x}$ & $\stackrel{\times}{\simeq}$ & $\underset{x}{x}$ & 离 & $\stackrel{2}{ \pm}$ & 8 & $\underset{\square}{x}$ & x & 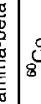 & 3 & ${ }_{8}^{\circ}$ & $\frac{E}{\bar{\alpha}}$ & $\mathrm{g}^{\frac{2}{2}}$ \\
\hline 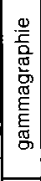 & 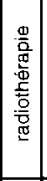 & & 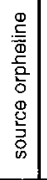 & 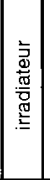 & 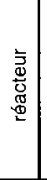 & 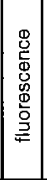 & & 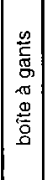 & & 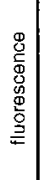 & 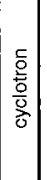 & & & & & & 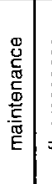 & 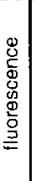 & & & & & 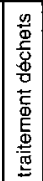 & 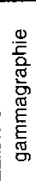 \\
\hline- & $\Sigma$ & - & $\cdots$ & - & $\sigma$ & - & - & 匹 & $\Sigma$ & - & $\pi$ & $a$ & $\sigma$ & - & $\mathrm{u}$ & - & $\Sigma$ & 匹ᄄ & - & - & - & $a$ & $\frac{0}{z}$ & - \\
\hline & & 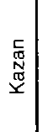 & 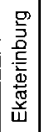 & & 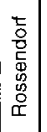 & & & $\frac{\underline{y}}{\frac{y}{\pi}}$ & & $\frac{\mathscr{\Phi}}{\underline{\underline{\underline{T}}}}$ & 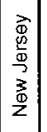 & 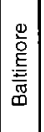 & & & & & $\underset{\underline{N}}{\stackrel{\bar{E}}{~}}$ & $\frac{\frac{\omega}{0}}{\overline{10}}$ & $\begin{array}{l}\vec{z} \\
0 \\
o \\
z \\
\Sigma \\
\Sigma\end{array}$ & & $\begin{array}{ll} \\
\end{array}$ & 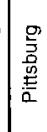 & 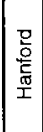 & \begin{tabular}{|l}
$\frac{0}{0}$ \\
$\frac{0}{0}$ \\
$\frac{3}{2}$ \\
0 \\
0 \\
0
\end{tabular} \\
\hline 惫 & 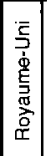 & $\begin{array}{l}n \\
0 \\
\frac{0}{د} \\
د\end{array}$ & $\mid \begin{array}{l}0 \\
0 \\
\frac{1}{5} \\
0\end{array}$ & $\mid \begin{array}{l}n \\
\mathscr{D} \\
\frac{1}{5}\end{array}$ & 㔛 & 选 & 䞨 & 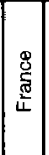 & 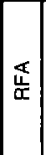 & $\mathbb{\widetilde { x }}$ & 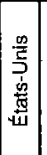 & 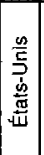 & 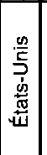 & $\underset{\mathbb{4}}{\mathbb{4}}$ & 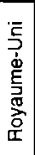 & 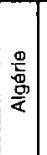 & $\begin{array}{l}\frac{0}{\bar{z}} \\
\frac{\vec{g}}{\mathrm{~g}} \\
\end{array}$ & 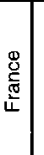 & 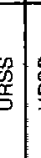 & 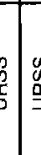 & $\frac{8}{5}$ & 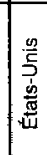 & 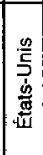 & 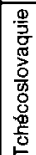 \\
\hline 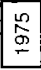 & \begin{tabular}{|l|} 
\\
0 \\
0 \\
$\tau$
\end{tabular} & $\begin{array}{l}\stackrel{2}{\alpha} \\
\sigma \\
\sigma\end{array}$ & 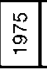 & $\begin{array}{l}\stackrel{\sim}{\Omega} \\
\sigma \\
\sigma\end{array}$ & $\left|\begin{array}{l}m \\
0 \\
\sigma \\
\sigma \\
\end{array}\right|$ & $\left|\begin{array}{l}\frac{m}{2} \\
\sigma \\
\sigma \\
\sigma\end{array}\right|$ & \begin{tabular}{|l}
$\frac{10}{5}$ \\
0 \\
$\Gamma$
\end{tabular} & 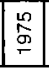 & 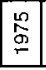 & 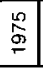 & $\left|\begin{array}{l}0 \\
0 \\
0 \\
\end{array}\right|$ & $\mid \begin{array}{l}0 \\
\stackrel{0}{\sigma} \\
-\end{array}$ & $\mid \begin{array}{l}0 \\
\stackrel{0}{\sigma} \\
\stackrel{2}{\sigma}\end{array}$ & 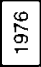 & $\begin{array}{l}0 \\
0 \\
0 \\
0\end{array}$ & $\left|\begin{array}{l}0 \\
0 \\
0 \\
\sigma\end{array}\right|$ & $\begin{array}{l}0 \\
\stackrel{0}{0} \\
-\end{array}$ & $\begin{array}{l}0 \\
\stackrel{0}{0} \\
\stackrel{2}{2}\end{array}$ & $\begin{array}{l}0 \\
\stackrel{0}{9} \\
\stackrel{2}{2}\end{array}$ & 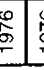 & 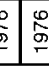 & 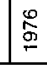 & 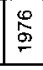 & 今ิ \\
\hline
\end{tabular}




\begin{tabular}{|c|c|c|c|c|c|c|c|c|c|c|c|c|c|c|c|c|c|c|c|c|c|c|c|}
\hline 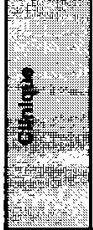 & 商 & & 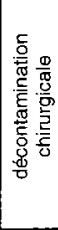 & & & 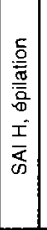 & 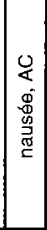 & & & & 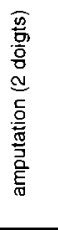 & & $\begin{array}{l}\frac{I}{d} \\
\bar{d}\end{array}$ & & & & & 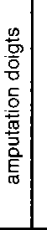 & & 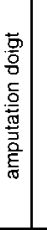 & & & \\
\hline 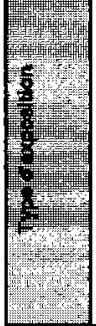 & 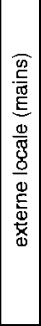 & 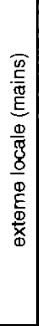 & 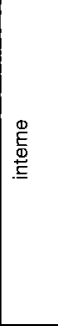 & 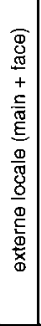 & 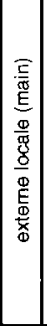 & 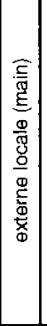 & 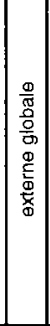 & 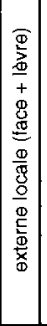 & 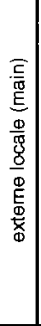 & 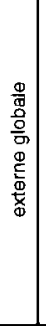 & 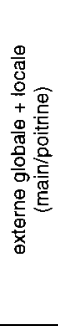 & & 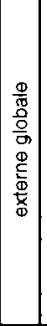 & 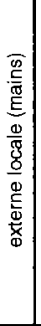 & 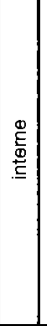 & & 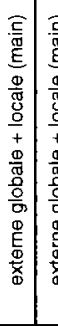 & 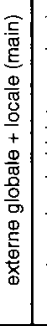 & 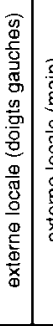 & 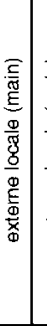 & 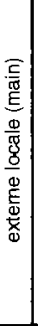 & 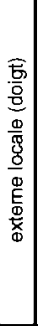 & 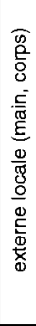 \\
\hline$\frac{1}{9}=$ & $\circ$ & 0 & 0 & - & 0 & 0 & 0 & 0 & 10 & 0 & 0 & 0 & 0 & 0 & 0 & $\circ$ & $\circ$ & 0 & $\circ$ & 0 & 0 & 0 & 0 \\
\hline & - & $m$ & $r$ & - & - & - & $1-$ & -1 & $r$ & - & $\tau$ & - & - & $\Gamma$ & $\sim$ & $\sim$ & $r$ & 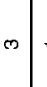 & - & - & $N$ & $r$ & $\sim$ \\
\hline & $\underset{x}{x}$ & $\stackrel{\text { শ }}{\underset{x}{2}}$ & & 짐 & 8 & 8 & $\approx$ & 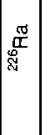 & 0 & $\begin{array}{l}8 \\
8 \\
8\end{array}$ & $\underline{\underline{m}}$ & $a^{2}$ & $\stackrel{8}{8}$ & $\begin{array}{l}\frac{0}{5} \\
\frac{0}{0} \\
\frac{5}{2}\end{array}$ & E & 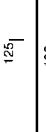 & 竞 & 褢 & $\stackrel{2}{*}$ & $\stackrel{\underline{\underline{w}}}{\underline{\underline{2}}}$ & ख્a & $\underset{\mathbb{C}}{x}$ & $\underset{x}{x}$ \\
\hline $\begin{array}{l}8 \\
8 \\
8\end{array}$ & & 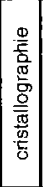 & 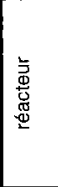 & & & 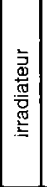 & 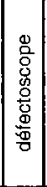 & 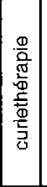 & & 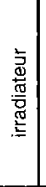 & 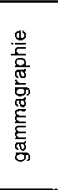 & & 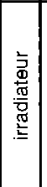 & 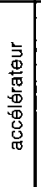 & 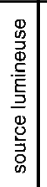 & & 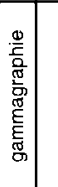 & & & 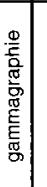 & & & 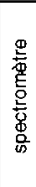 \\
\hline & $\sim$ & - & $\frac{y}{2}$ & $\sim$ & - & - & $1-$ & $\Sigma$ & $\infty$ & - & - & $\Sigma$ & - & $\approx$ & $-m$ & $\Sigma$ & - & - & -- & - & on & $r$ & - \\
\hline $\begin{array}{l}4 \\
+19\end{array}$ & 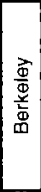 & 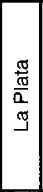 & $\frac{\pi}{9}$ & & 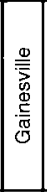 & 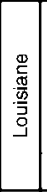 & : & $\begin{array}{l}\frac{c}{\bar{t}} \\
\stackrel{\bar{\sigma}}{\circ}\end{array}$ & 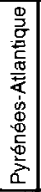 & 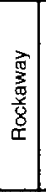 & 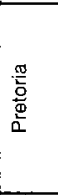 & 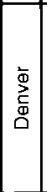 & 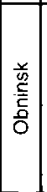 & $\stackrel{\vec{\Phi}}{\bar{y}}$ & & & & $\begin{array}{l}\frac{0}{0} \\
\frac{3}{0} \\
\frac{0}{0}\end{array}$ & 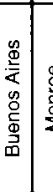 & 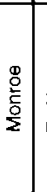 & 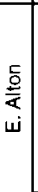 & 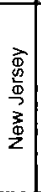 & $\begin{array}{l}\text { 离 } \\
\frac{0}{0} \\
\frac{0}{2} \\
\frac{3}{3} \\
\frac{1}{2} \\
2\end{array}$ \\
\hline $\begin{array}{l}4 \\
4 \\
4\end{array}$ & 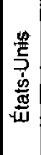 & 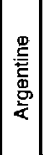 & 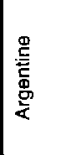 & 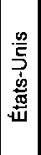 & 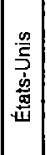 & 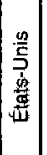 & 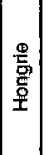 & 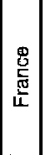 & $\begin{array}{l}\mathrm{g} \\
\mathrm{g} \\
\frac{\mathrm{v}}{\mathrm{L}} \\
\mathrm{L}\end{array}$ & 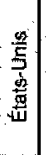 & $\begin{array}{l}\frac{0}{0} \\
0 \\
\frac{3}{5} \\
9 \\
\frac{3}{9} \\
\frac{9}{4}\end{array}$ & 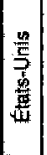 & $\begin{array}{l}0 \\
0 \\
0 \\
5 \\
5\end{array}$ & $\begin{array}{l}\text { 盟 } \\
\frac{\text { In }}{5}\end{array}$ & 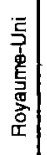 & 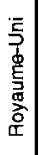 & 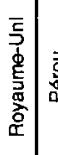 & 总 & : & 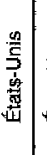 & 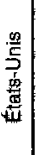 & 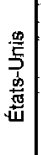 & 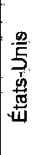 \\
\hline$\delta$ & $\hat{~}$ & 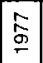 & 令 & $\stackrel{0}{\sigma}$ & $\mid \begin{array}{l}\hat{0} \\
\frac{0}{2}\end{array}$ & \begin{tabular}{|l|} 
\\
$\vdots$ \\
0 \\
\end{tabular} & $\mid \begin{array}{l}5 \\
\\
\end{array}$ & \begin{tabular}{|l|} 
\\
0 \\
0 \\
\end{tabular} & 命 & $\begin{array}{l}\text { E } \\
\text { के }\end{array}$ & $\begin{array}{l}E \\
\text { O } \\
\sigma\end{array}$ & \begin{tabular}{l}
$\hat{E}$ \\
0 \\
\hdashline
\end{tabular} & $\mid \begin{array}{l}5 \\
\frac{1}{\sigma} \\
\end{array}$ & $\begin{array}{l}\hat{\sigma} \\
\hat{\sigma}\end{array}$ & $\begin{array}{l}\hat{f} \\
\stackrel{6}{\circ} \\
\end{array}$ & 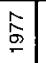 & \begin{tabular}{l|l}
$E$ & 1 \\
0 & 0 \\
& 0
\end{tabular} & 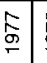 & 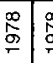 & $\begin{array}{l}\stackrel{\infty}{0} \\
\stackrel{0}{\sigma} \\
\stackrel{1}{*}\end{array}$ & $\begin{array}{c}\infty \\
\stackrel{\infty}{+} \\
\stackrel{2}{+}\end{array}$ & 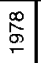 & $\begin{array}{l}\frac{\infty}{2} \\
\vdots \\
\sigma \\
\end{array}$ \\
\hline
\end{tabular}




\begin{tabular}{|c|c|c|c|c|c|c|c|c|c|c|c|c|c|c|c|c|c|c|c|c|c|c|c|}
\hline & & & 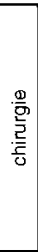 & & 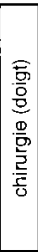 & 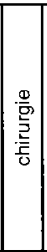 & 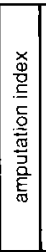 & & & & & & & & & 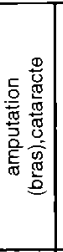 & 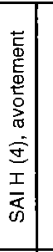 & & & 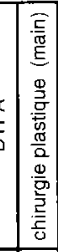 & & & \\
\hline 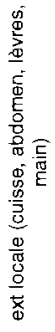 & 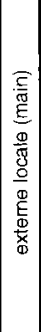 & 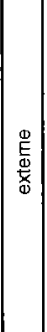 & 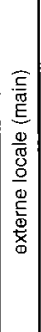 & 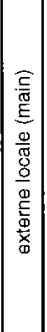 & 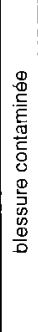 & 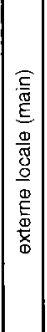 & 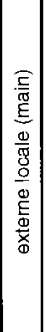 & 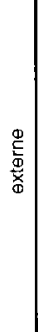 & 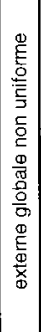 & 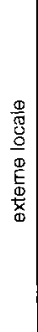 & 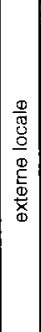 & 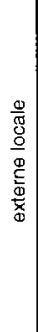 & 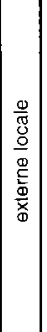 & 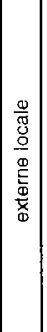 & 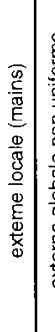 & 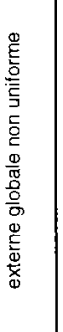 & 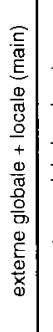 & 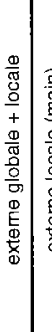 & 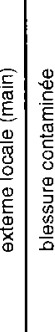 & 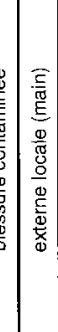 & 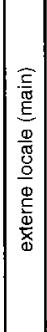 & 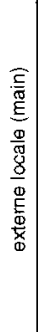 & 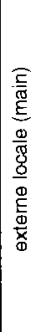 \\
\hline 0 & 0 & 0 & 0 & 0 & 0 & 0 & 0 & 0 & 0 & 0 & 0 & 0 & 0 & 0 & $\circ$ & 0 & 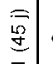 & $\circ$ & $\circ \quad 0$ & 0 & 0 & 0 & 0 \\
\hline$r$ & $r$ & - & $\sim$ & I & 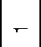 & - & - & - & - & - & - & - & -1 & - & - & $\infty$ & $\mathbb{N}$ & $\stackrel{D}{\mathrm{~N}}$. & $\sim$ & $v-$ & - & - & - \\
\hline$\omega$ & 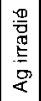 & 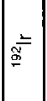 & 8 & æx & $\mid \begin{array}{l}\frac{E}{5} \\
\vdots \\
\vdots \\
\end{array}$ & 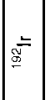 & 爻 & $\stackrel{\bar{g}}{\underline{g}}$ & & $\mathrm{~S}^{2}$ & $\Phi^{2}$ & $\begin{array}{l}\frac{0}{5} \\
\frac{0}{0} \\
\frac{0}{2}\end{array}$ & 畄 & $\Phi$ & $\begin{array}{l}\frac{D}{5} \\
\frac{0}{ \pm} \\
\frac{w}{w}\end{array}$ & $\begin{array}{l}\frac{E}{\bar{z}} \\
\text { 产 } \\
\frac{3}{\alpha}\end{array}$ & $\frac{2}{\sigma_{\Phi}^{2}}$ & $\begin{array}{l}3 \\
0 \\
0\end{array}$ & $\begin{array}{l}\text { 是 } \\
\text { 员 } \\
\text { 言 }\end{array}$ & $\bar{E}$ & $\underset{x}{x}$ & 8 & $\stackrel{2}{\Phi^{2}}$ \\
\hline 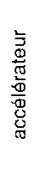 & 言 & 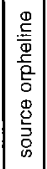 & 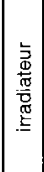 & 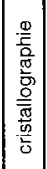 & & & & 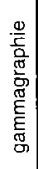 & 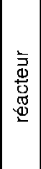 & 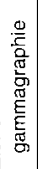 & 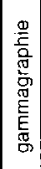 & 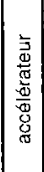 & & 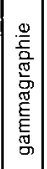 & 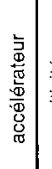 & & 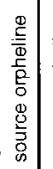 & 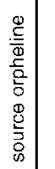 & 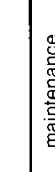 & 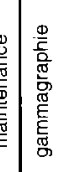 & & & \\
\hline$\check{x}$ & $\pi$ & - & $\approx$ & - & $\bar{\Sigma}$ & - & - & - & 따 & - & - & $\approx$ & $\propto$ & - & $\pi$ & $\mathrm{O}$ & - & $\sim 0$ & $\sim \bar{z}$ & $\bar{\Sigma}-$ & ᄄ & $\therefore$ & - \\
\hline & & & $\mid \begin{array}{l}\frac{n}{2} \\
\frac{1}{0} \\
0\end{array}$ & 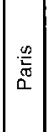 & 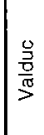 & $\mid \begin{array}{l}\frac{0}{L} \\
\frac{0}{\sigma 0} \\
0\end{array}$ & $\begin{array}{l}\vec{z} \\
\bar{w} \\
z\end{array}$ & & $\begin{array}{l}\frac{\text { क }}{2} \\
\frac{2}{2} \\
\frac{8}{2}\end{array}$ & 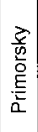 & 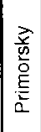 & 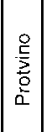 & 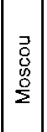 & 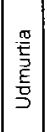 & 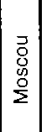 & 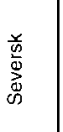 & $\begin{array}{l}\text { 离 } \\
\text { 足 }\end{array}$ & $\begin{array}{l}\frac{5}{\mathbb{T}} \\
\frac{0}{1} \\
\frac{0}{1}\end{array}$ & & 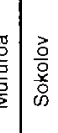 & & $\frac{\infty}{2}$ & 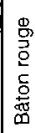 \\
\hline 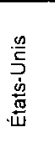 & 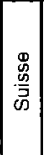 & $\mid \begin{array}{l}\frac{8}{0} \\
\frac{\pi}{2}\end{array}$ & 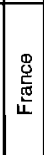 & 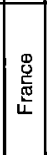 & 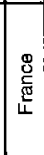 & 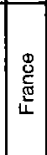 & 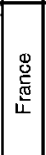 & 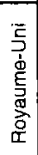 & 号 & $\begin{array}{l}y \\
0 \\
5 \\
5\end{array}$ & $\mid \begin{array}{l}\mathscr{2} \\
\text { 采 } \\
\end{array}$ & $\begin{array}{l}n \\
\mathscr{n} \\
\text { 点 }\end{array}$ & 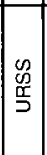 & 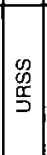 & $\mid \begin{array}{l}\mathscr{y} \\
0 \\
\frac{0}{5} \\
5\end{array}$ & $\begin{array}{l}\mathscr{D} \\
\frac{0}{5} \\
5\end{array}$ & $\begin{array}{l}\frac{0}{0} \\
\frac{5}{2} \\
\frac{2}{2}\end{array}$ & 昜 & 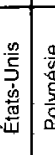 & 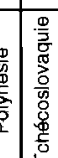 & 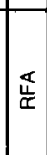 & 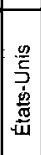 & 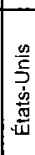 \\
\hline 兽 & \begin{tabular}{|l|} 
\\
$\stackrel{\infty}{\sigma}$ \\
$\stackrel{\sigma}{\sigma}$
\end{tabular} & \begin{tabular}{|l|}
$\stackrel{\infty}{a}$ \\
$\stackrel{\sigma}{\sigma}$ \\
\end{tabular} & \begin{tabular}{|l}
$\frac{D}{2}$ \\
$\stackrel{5}{\sigma}$
\end{tabular} & \begin{tabular}{|l|}
$\mathbf{a}$ \\
$\stackrel{0}{\sigma}$ \\
\end{tabular} & 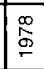 & \begin{tabular}{|l|l} 
\\
$\stackrel{2}{\sigma}$ \\
$\stackrel{9}{\sigma}$
\end{tabular} & \begin{tabular}{|l|}
$\infty$ \\
\multirow{2}{\sigma}{} \\
\end{tabular} & 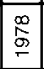 & \begin{tabular}{|l}
$\infty$ \\
$\stackrel{2}{\sigma}$ \\
$\sigma$ \\
$\Gamma$
\end{tabular} & \begin{tabular}{|l|}
$\infty$ \\
0 \\
0 \\
0 \\
\end{tabular} & 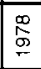 & 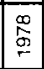 & \begin{tabular}{|l|}
$\infty$ \\
$\stackrel{0}{\circ}$ \\
\end{tabular} & 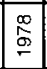 & 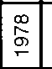 & 悉 & \begin{tabular}{|l|l|}
$\begin{array}{l}0 \\
0 \\
0 \\
\end{array}$ \\
\end{tabular} & 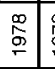 & 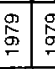 & 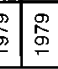 & \begin{tabular}{|l|}
\multirow{2}{9}{} \\
$\stackrel{9}{9}$
\end{tabular} & \begin{tabular}{|l|} 
\\
0 \\
0 \\
\end{tabular} & \begin{tabular}{|l}
9 \\
\\
0 \\
0
\end{tabular} \\
\hline
\end{tabular}




\begin{tabular}{|c|c|c|c|c|c|c|c|c|c|c|c|c|c|c|c|c|c|c|c|c|c|c|}
\hline$\frac{9}{d y}$ & 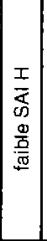 & 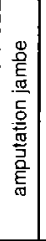 & & & & & 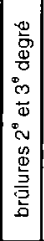 & 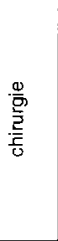 & 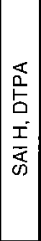 & & & & $\bar{\sigma}$ & & ळ & & & 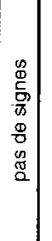 & 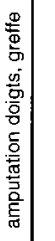 & & 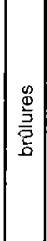 & 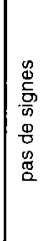 \\
\hline 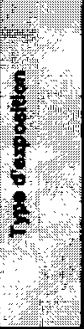 & 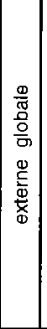 & 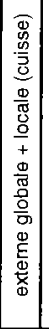 & 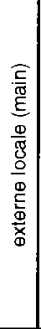 & 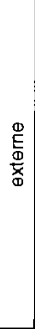 & 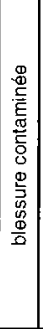 & 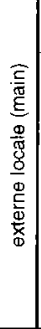 & 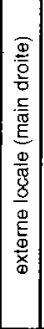 & 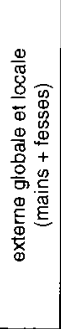 & 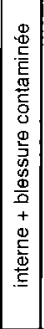 & 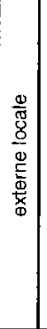 & 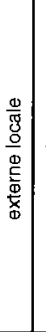 & 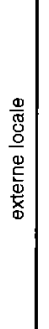 & 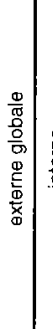 & 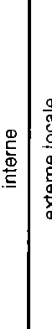 & 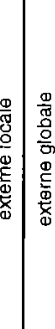 & 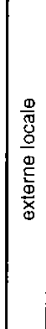 & 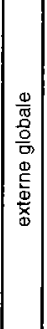 & 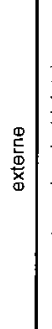 & 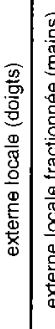 & 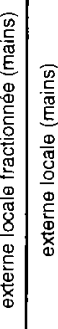 & 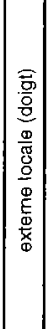 & 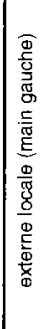 \\
\hline 8 & 。 & 0 & 0 & 0 & 0 & $\circ$ & 0 & 0 & 0 & 0 & 0 & 0 & 0 & $\circ$ & 0 & 0 & - & 0 & 00 & $\begin{array}{lll}0 & 0\end{array}$ & 0 & 0 \\
\hline $\begin{array}{l}89 \\
67 \\
2\end{array}$ & - & - & - & - & - & - & -1 & $\mp$ & $r$ & - & $\sim$ & -1 & $-{ }^{\circ}$ & $\infty$ & 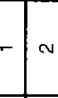 & - & - & $\infty$ & - & $\sim|c| c$ & - & - \\
\hline$y^{2}$ & $\underset{\propto}{\times}$ & \pm & 蛋 & & $\begin{array}{l}\text { 틀 } \\
\frac{\bar{t}}{\mathrm{D}} \\
\text { 言 }\end{array}$ & $x$ & $\underset{x}{x}$ & 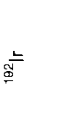 & 념 & 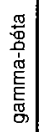 & 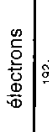 & $\underset{\sigma}{2}$ & $\therefore$ & ร- & x) & $\Phi_{\Phi}^{\underline{4}}$ & $\because$ & \begin{tabular}{l|l}
$\underset{\mathrm{g}}{\mathrm{E}}$ \\
$\stackrel{\mathrm{E}}{\mathrm{G}}$ \\
$\mathrm{d}$
\end{tabular} & $\frac{2}{\sigma^{2}}$ & $\stackrel{x}{x}$ & $\underset{\mathbb{x}}{\stackrel{\times}{x}}$ & के \\
\hline$\frac{6}{4}$ & 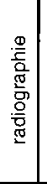 & 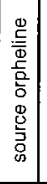 & & 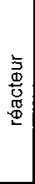 & 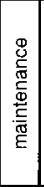 & 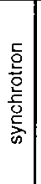 & 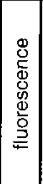 & 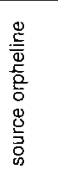 & & & 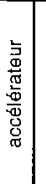 & 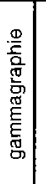 & & & 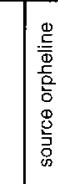 & 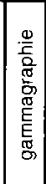 & 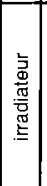 & $\begin{array}{l}\frac{0}{0} \\
\frac{0}{0} \\
\frac{0}{0} \\
\frac{0}{0} \\
\frac{0}{0} \\
\frac{0}{2}\end{array}$ & & 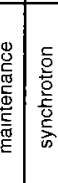 & & 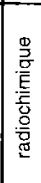 \\
\hline 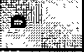 & $\Sigma$ & - & - & $\alpha$ & $\frac{0}{2}$ & $\simeq$ & - & - & - & $\stackrel{0}{2}$ & $\approx$ & - & $-1=$ & $\Sigma-$ & $-1-$ & - & - & $\Sigma$ & -1 & \begin{tabular}{l|c} 
& \multicolumn{1}{|l}{}
\end{tabular} & - & $\pi$ \\
\hline 3 & 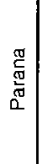 & 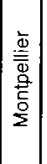 & 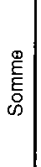 & & 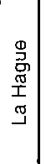 & 点 & 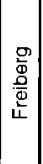 & 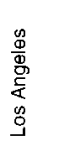 & & 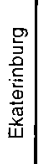 & 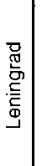 & 畩 & 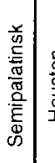 & 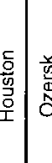 & $\underset{⿱ 亠}{\stackrel{O}{N}}$ & 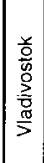 & 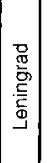 & 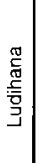 & & 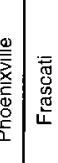 & & 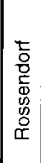 \\
\hline (5) & 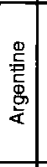 & 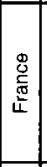 & 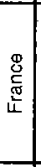 & $\begin{array}{l}\mathbb{8} \\
\substack{\mathbb{N} \\
\mathbb{L}}\end{array}$ & 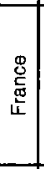 & 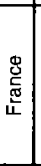 & 递 & 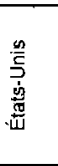 & $\frac{\text { 焉 }}{\frac{1}{5}}$ & $\begin{array}{l}\mathscr{\mathscr { B }} \\
\underline{x} \\
\underline{5}\end{array}$ & 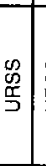 & 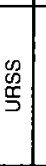 & 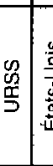 & | & 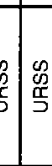 & $\begin{array}{l}\mathscr{y} \\
0 \\
\frac{0}{5} \\
\end{array}$ & $\begin{array}{l}\infty \\
0 \\
\frac{2}{5} \\
5\end{array}$ & 혼 & 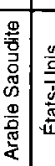 & 恣 & $\begin{array}{l}\mathbb{d} \\
\mathbb{x}\end{array}$ & $\underset{0}{\alpha}$ \\
\hline$f^{4}$ & $\begin{array}{l}\stackrel{9}{8} \\
\stackrel{9}{0} \\
\end{array}$ & \begin{tabular}{|l|}
0 \\
0 \\
0 \\
-1
\end{tabular} & $\begin{array}{c}\stackrel{9}{0} \\
\stackrel{9}{9} \\
\end{array}$ & 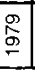 & $\begin{array}{l}9 \\
0 \\
0 \\
-1\end{array}$ & $\begin{array}{l}\stackrel{8}{8} \\
\stackrel{5}{-}\end{array}$ & $\begin{array}{l}\frac{9}{2} \\
\hat{\sigma} \\
+\end{array}$ & 总 & 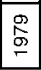 & 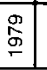 & $\begin{array}{l}0 \\
0 \\
0 \\
\end{array}$ & 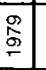 & \begin{tabular}{l|l}
9 & \\
0 & \\
& \\
\end{tabular} & 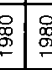 & 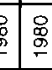 & \begin{tabular}{|l|} 
\\
$\stackrel{\$}{\circ}$ \\
\end{tabular} & \begin{tabular}{|l|}
\multirow{2}{\circ}{} \\
$\stackrel{\circ}{\circ}$ \\
\end{tabular} & 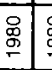 & 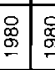 & $\begin{array}{l}0 \\
0 \\
\end{array}$ & 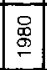 & $\underset{8}{\stackrel{\circ}{\circ}}$ \\
\hline
\end{tabular}




\begin{tabular}{|c|c|c|c|c|c|c|c|c|c|c|c|c|c|c|c|c|c|c|c|c|c|c|c|c|c|}
\hline & 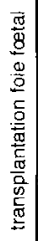 & & & & & & & & & 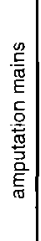 & 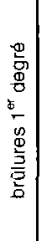 & $\begin{array}{l} \\
\frac{0}{9} \\
\frac{2}{2} \\
\frac{2}{5} \\
0\end{array}$ & 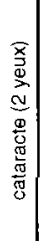 & 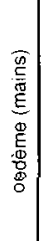 & 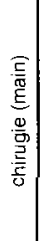 & & & & & 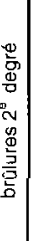 & & & & & $\bar{\delta}$ \\
\hline 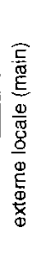 & 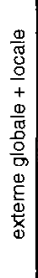 & 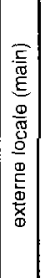 & 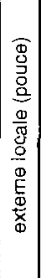 & 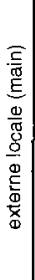 & 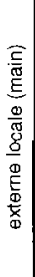 & 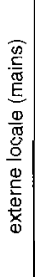 & 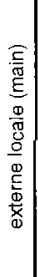 & 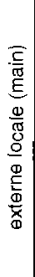 & 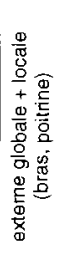 & 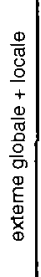 & 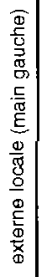 & 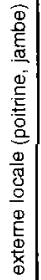 & 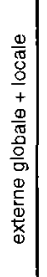 & 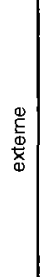 & 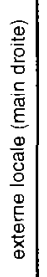 & 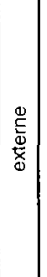 & 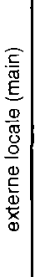 & 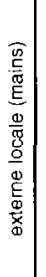 & & 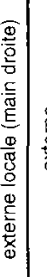 & & 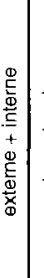 & 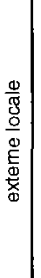 & 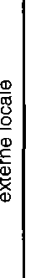 & 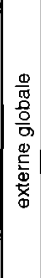 \\
\hline 0 & 0 & 0 & 0 & $\circ$ & $\circ$ & 0 & $\circ$ & 0 & & 0 & 0 & 0 & 0 & 0 & $\circ$ & 0 & 0 & $\circ$ & $\stackrel{2}{\stackrel{2}{2}}$ & 。 & 0 & $\circ$ & 0 & $\circ$ & 0 \\
\hline N & - & - & - & - & -1 & $\mathrm{v}$ & - & - & - & $=$ & - & - & - & - & - & - & - & - & - & - & $\cdots$ & $\begin{array}{l}0 \\
0 \\
0\end{array}$ & $\underset{\wedge}{\mathbb{N}}$ & + & $=$ \\
\hline xִ & 8 & 8 & 쯤 & $\underset{x}{x}$ & $\stackrel{x}{ᄄ x}$ & 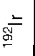 & $\underset{\widetilde{c}}{x}$ & 조 & $\frac{2}{2}$ & 8 & \begin{tabular}{|l|}
$\underset{x}{x}$ \\
\end{tabular} & $\frac{2}{s_{0}^{2}}$ & $\underset{\underset{x}{x}}{\times}$ & 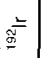 & $\underbrace{2}_{\infty}$ & $\begin{array}{l}x \\
\mathbb{E}\end{array}$ & $\frac{2}{2}$ & $\underset{x}{x}$ & 8 & $\underset{\widetilde{a}}{x}$ & 8 & $\frac{E}{5}$ & $\frac{2}{2}$ & $=$ & $\frac{8}{8}$ \\
\hline 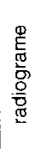 & 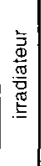 & & 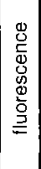 & & & & & 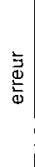 & 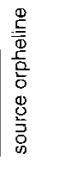 & 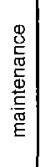 & 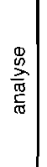 & 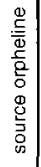 & 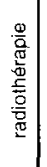 & 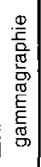 & 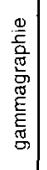 & 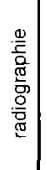 & 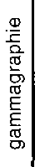 & 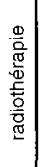 & 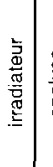 & 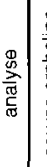 & 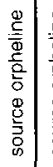 & 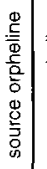 & 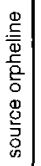 & 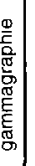 & छ气 \\
\hline- & - & - & - & $\therefore$ & 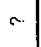 & - & $\sim$ & $\cdots$ & - & $\Sigma$ & $\propto$ & - & $\Sigma$ & - & - & $\Sigma$ & - & $\Sigma$ & -0 & 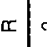 & $\cdots$ & - & - & - & a. \\
\hline & 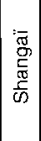 & 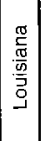 & & & & 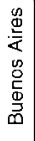 & & 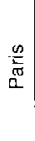 & $\begin{array}{l}\frac{\mathrm{w}}{\mathrm{E}} \\
\frac{\mathrm{O}}{\mathrm{\sigma}} \\
\frac{\mathrm{\sigma}}{\mathrm{g}}\end{array}$ & 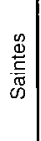 & 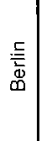 & 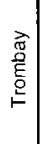 & 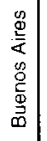 & $\begin{array}{l}\frac{x}{\pi} \\
\frac{\pi}{0} \\
\mathbb{D} \\
\end{array}$ & 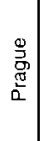 & & & 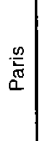 & $\frac{\frac{5}{\underline{\underline{w}}}}{\frac{\underline{\underline{m}}}{x}}$ & $\begin{array}{l}\frac{c}{\bar{G}} \\
\mathbb{D}\end{array}$ & 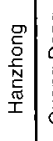 & 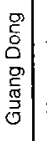 & 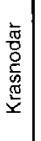 & 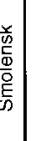 & 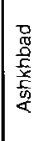 \\
\hline$\underset{\frac{\alpha}{\alpha}}{\mathbb{\alpha}}$ & 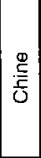 & $\mid \begin{array}{c}\frac{\omega}{5} \\
5 \\
\dot{d} \\
\frac{w}{\tilde{w}} \\
\end{array}$ & 岳 & 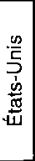 & $\begin{array}{l}\frac{\infty}{5} \\
5 \\
\frac{\phi}{p} \\
\frac{m}{4}\end{array}$ & 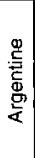 & 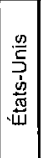 & $\begin{array}{l}\mathbb{8} \\
\stackrel{\mathbb{Z}}{\pi} \\
\frac{\pi}{4}\end{array}$ & 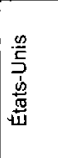 & 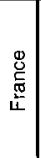 & 咅 & 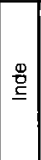 & 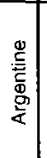 & 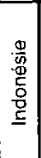 & 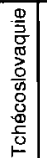 & 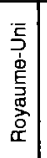 & $\begin{array}{l}0 \\
\stackrel{0}{\circ} \\
0 \\
0\end{array}$ & 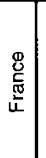 & 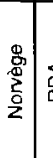 & 遌 & 密 & 竞 & $\begin{array}{l}\text { 足 } \\
\frac{\underline{L}}{5}\end{array}$ & 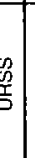 & 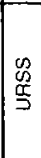 \\
\hline ম్ల & 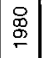 & 商 & $\stackrel{\text { s. }}{\stackrel{\sigma}{\sigma}}$ & $\begin{array}{l}\mathbf{\infty} \\
\stackrel{\sigma}{\sigma}\end{array}$ & 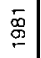 & $\bar{D}$ & 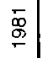 & 㠃 & 变 & 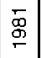 & 产 & $\begin{array}{l}\mathscr{\infty} \\
\stackrel{\leftrightarrow}{\rightarrow}\end{array}$ & \begin{tabular}{|l}
$\stackrel{\mathscr{\alpha}}{\circ}$ \\
$\stackrel{\circ}{\circ}$
\end{tabular} & 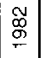 & 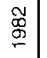 & $\begin{array}{l}\text { 吅 } \\
\leftarrow\end{array}$ & $\begin{array}{l}\stackrel{\sim}{\infty} \\
\stackrel{\sigma}{\sigma}\end{array}$ & 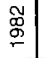 & 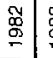 & \% & 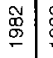 & 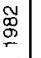 & 䓵 & 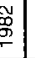 & \begin{tabular}{|l}
$\widetilde{o}$ \\
o.
\end{tabular} \\
\hline
\end{tabular}




\begin{tabular}{|c|c|c|c|c|c|c|c|c|c|c|c|c|c|c|c|c|c|c|c|c|c|c|c|c|}
\hline & $\overline{\boldsymbol{\sigma}}$ & $\bar{\varpi}$ & & & 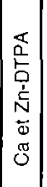 & 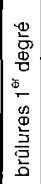 & 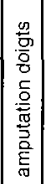 & 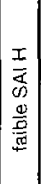 & $\mid \begin{array}{c}z \\
0 \\
0 \\
0 \\
+ \\
+ \\
\frac{1}{a} \\
\bar{a} \\
\omega\end{array}$ & & & & & & 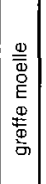 & & & & & & 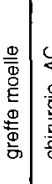 & & 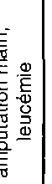 & \\
\hline 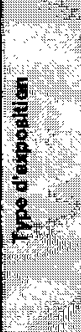 & 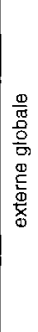 & 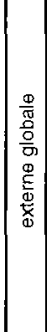 & 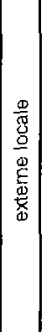 & 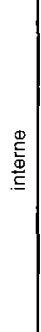 & 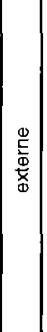 & 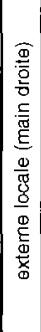 & 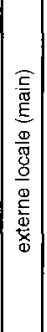 & 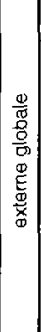 & 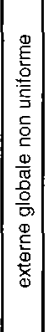 & 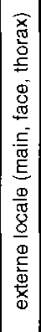 & 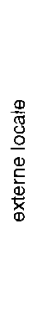 & 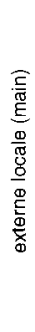 & 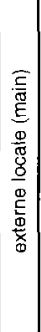 & 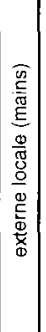 & 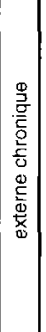 & 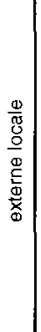 & 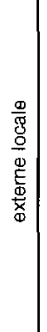 & 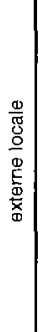 & 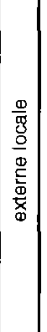 & 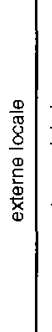 & 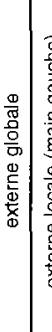 & 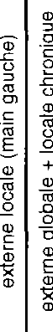 & 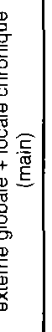 & 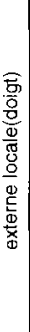 \\
\hline$\frac{8}{8}$ & $\mathrm{c}$ & in & 0 & 0 & 0 & 0 & 0 & $\circ$ & 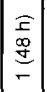 & 0 & 0 & 0 & 0 & $\circ$ & 0 & 0 & 0 & 0 & 0 & 0 & 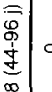 & 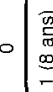 & & 0 \\
\hline$y^{2}$ & 6 & ะ & $\sim$ & - & -1 & $1-$ & - & $\sim$ & 0 & - & - & - & - & - & 8 & -1 & - & - & - & - & $\stackrel{N}{N}$ & - & & - \\
\hline & 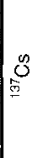 & 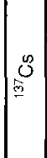 & $\stackrel{*}{*}$ & $\begin{array}{c}E \\
\frac{E}{\alpha} \\
\frac{E}{\sigma}\end{array}$ & $\mid \frac{E}{5}$ & $\stackrel{2}{2}$ & $=$ & $\stackrel{8}{8}$ & 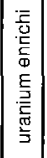 & 점 & $\underset{\text { x }}{x}$ & 중 & $\underset{\mathbb{x}}{\times}$ & 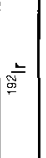 & 8 & 㜽 & $\begin{array}{l}0 \\
0 \\
0 \\
0\end{array}$ & $\begin{array}{l}\text { 踣 } \\
\end{array}$ & \begin{tabular}{|l|}
0 \\
0 \\
0 \\
0
\end{tabular} & 䇏 & $\stackrel{2}{2}$ & $\frac{2}{2}$ & & 낳 \\
\hline 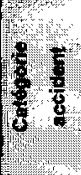 & 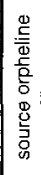 & 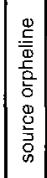 & 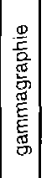 & & 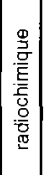 & & & 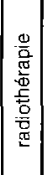 & 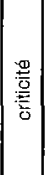 & 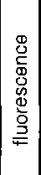 & & 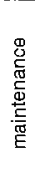 & & 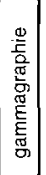 & 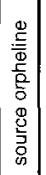 & & 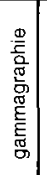 & 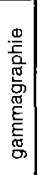 & 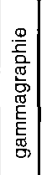 & 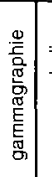 & 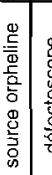 & 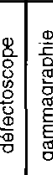 & & 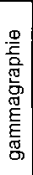 \\
\hline & c. & $\therefore$ & - & $\cdots$ & $\pi$ & - & - & $\Sigma$ & $\frac{0}{2}$ & $\approx$ & - & $\approx$ & $\cdots$ & - & $\Sigma$ & -1 & - & -1 & - & - & $-1-$ & - & & - \\
\hline is & 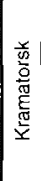 & 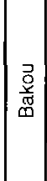 & 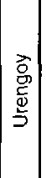 & 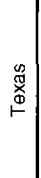 & $\begin{array}{l}\frac{\mathscr{N}}{\mathrm{N}} \\
\frac{\Phi}{\Phi}\end{array}$ & 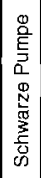 & 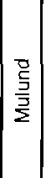 & 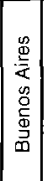 & 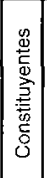 & & & 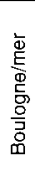 & 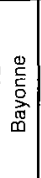 & & 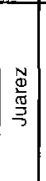 & $\begin{array}{l}\vec{z} \\
\bar{w} \\
\frac{0}{\Sigma} \\
\Sigma\end{array}$ & 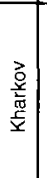 & 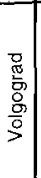 & $\stackrel{m}{5}$ & $\frac{\pi}{5}$ & 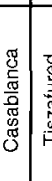 & 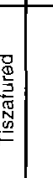 & & $\begin{array}{l}\mathbb{\pi} \\
\stackrel{D}{0} \\
\stackrel{D}{\tilde{D}} \\
\Sigma\end{array}$ \\
\hline$\frac{1}{2}$ & 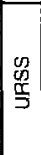 & 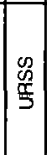 & $\begin{array}{l}0 \\
0 \\
\frac{0}{5} \\
5\end{array}$ & 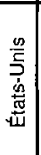 & 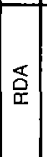 & 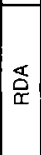 & $\stackrel{ }{\text { 을 }}$ & 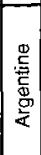 & 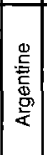 & 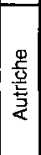 & $\underset{\mathbb{T}}{\mathbb{E}}$ & $\begin{array}{l}\text { g } \\
\stackrel{0}{c} \\
\text { 胥 } \\
\end{array}$ & 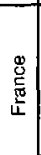 & $\underset{\mathbb{E}}{\stackrel{c}{\mathbb{E}}}$ & $\begin{array}{l}\frac{1}{3} \\
\frac{\bar{z}}{\bar{x}} \\
\frac{0}{2} \\
\end{array}$ & $\begin{array}{l}\mathscr{\mathscr { O }} \\
\stackrel{\mathscr{x}}{5}\end{array}$ & 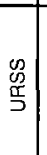 & $\begin{array}{l}0 \\
0 \\
\frac{0}{5}\end{array}$ & $\begin{array}{l}n \\
0 \\
\frac{n}{2} \\
د\end{array}$ & $\begin{array}{l}\mathscr{D} \\
\frac{D}{5} \\
\frac{5}{5}\end{array}$ & 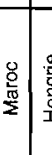 & 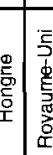 & & 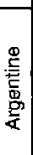 \\
\hline 8 & 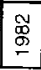 & 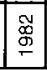 & \begin{tabular}{|l|}
$\tilde{a}$ \\
$\sigma$ \\
$\sigma$ \\
\end{tabular} & $\begin{array}{r}0 \\
0 \\
0 \\
0 \\
\end{array}$ & \begin{tabular}{|l|} 
\\
0 \\
0 \\
0 \\
-1 \\
\end{tabular} & 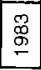 & \begin{tabular}{|l|} 
\\
0 \\
0 \\
\\
\end{tabular} & \begin{tabular}{|l|} 
\\
0 \\
0 \\
0 \\
\\
\end{tabular} & \begin{tabular}{|l|} 
\\
0 \\
0 \\
\\
\end{tabular} & \begin{tabular}{|l|}
0 \\
$\mathscr{8}$ \\
\hdashline \\
\end{tabular} & 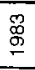 & 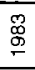 & $\begin{array}{l}0 \\
0 \\
\sigma \\
\end{array}$ & 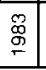 & 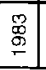 & 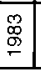 & 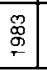 & 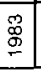 & $\begin{array}{l}0 \\
0 \\
0 \\
0 \\
\end{array}$ & $\begin{array}{l}0 \\
0 \\
0 \\
0 \\
\end{array}$ & 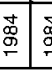 & 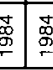 & & 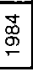 \\
\hline
\end{tabular}




\begin{tabular}{|c|c|c|c|c|c|c|c|c|c|c|c|c|c|c|c|c|c|c|c|c|c|c|c|}
\hline 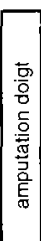 & 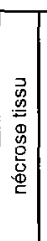 & & & & & & 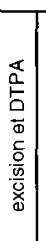 & & 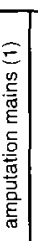 & & & & 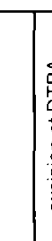 & & 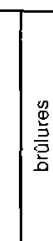 & 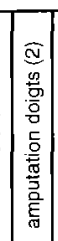 & & & & \begin{tabular}{|l}
$\frac{9}{\mathrm{~m}}$ \\
$\frac{\mathrm{E}}{\mathrm{c}}$ \\
$\frac{\mathrm{c}}{\mathrm{c}}$
\end{tabular} & & & 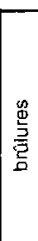 \\
\hline 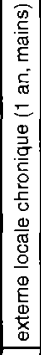 & 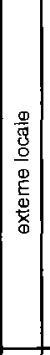 & 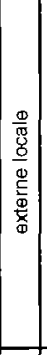 & 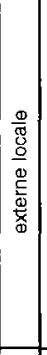 & 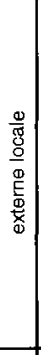 & 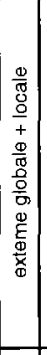 & 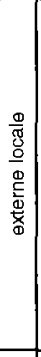 & & 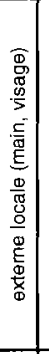 & 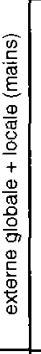 & 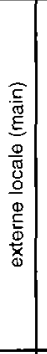 & 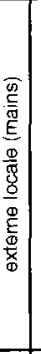 & 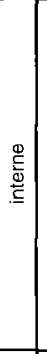 & 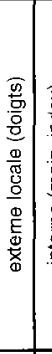 & 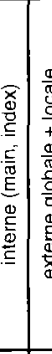 & 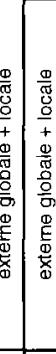 & 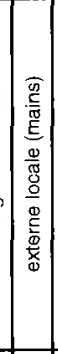 & 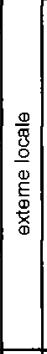 & & & 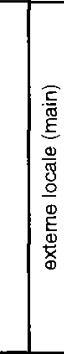 & | & 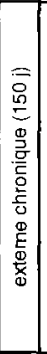 & 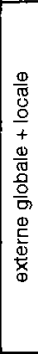 \\
\hline 0 & 0 & 0 & 0 & 0 & 0 & 0 & 0 & 0 & 01 & 0 & 0 & 0 & 0 & 0 & 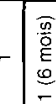 & 0 & 0 & 0 & 0 & 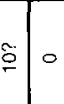 & - & 。 & $\begin{array}{l}\frac{\widehat{y}}{\bar{O}} \\
\underline{E} \\
\underline{\underline{m}} \\
\end{array}$ \\
\hline$\sim$ & $\infty$ & $\wedge$ & - & - & $\mp$ & - & - & - & $\sim$ & - & $\nabla$ & - & - & -10 & $\cdots$ & $\sim$ & $\infty$ & - & - & r & $\sim$ & $\infty$ & $\cdots$ \\
\hline${ }_{8}^{\circ}$ & 조 & $\underset{x}{x}$ & $\stackrel{2}{2}$ & $\frac{2}{2}$ & $\stackrel{2}{2}$ & $\begin{array}{c}\text { की } \\
\stackrel{0}{*}\end{array}$ & $\frac{E}{5}$ & $\underset{x}{x}$ & $\stackrel{2}{*}$ & 案 & $\frac{1}{2}$ & $\square$ & 竧 & 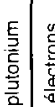 & 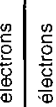 & $\frac{2}{9}$ & 8 & $\stackrel{2}{=}$ & 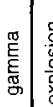 & 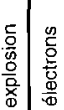 & 疋 & 品 & 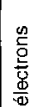 \\
\hline 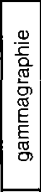 & 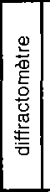 & 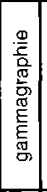 & & 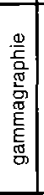 & & 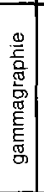 & & 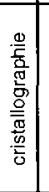 & 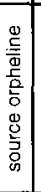 & & 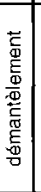 & & & 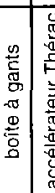 & 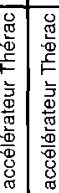 & 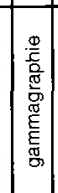 & & 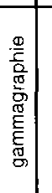 & & 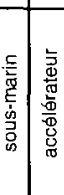 & 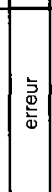 & 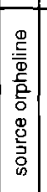 & 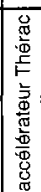 \\
\hline- & $\propto$ & -1 & - & - & - & - & -1 & - & - & - & -1 & $\Sigma$ & - & 0 & $\Sigma \Sigma$ & -1 & $\cdots$ & - & $\sim$ & $\overline{\mathbf{z}}-$ & $z$ & $\ldots$ & $z$ \\
\hline 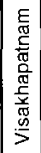 & 苞 & $\begin{array}{c}\frac{E}{\bar{\sigma}} \\
\bar{\alpha} \\
0\end{array}$ & 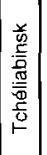 & $\stackrel{\pi}{5}$ & $\begin{array}{l}\bar{y} \\
0 \\
0\end{array}$ & $\begin{array}{l}0 \\
\frac{0}{2} \\
\frac{1}{1} \\
\frac{0}{2}\end{array}$ & 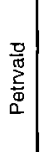 & & & & & & & 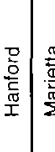 & 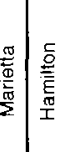 & 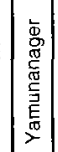 & 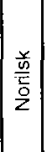 & 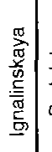 & 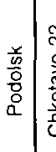 & 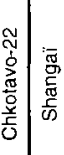 & & 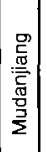 & 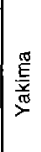 \\
\hline 离 & 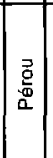 & $\begin{array}{l}\text { 呆 } \\
\text { 罂 }\end{array}$ & 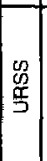 & $\begin{array}{l}\frac{n}{0} \\
\frac{0}{5} \\
\frac{1}{5}\end{array}$ & $\begin{array}{l}0,8 \\
\frac{8}{5}\end{array}$ & $\begin{array}{l}0 \\
0 \\
\frac{0}{5} \\
5\end{array}$ & 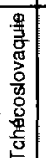 & 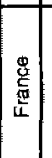 & 离 & 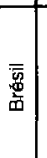 & 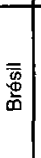 & 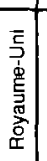 & 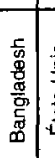 & 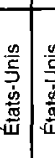 & 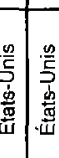 & $\frac{\text { D }}{\mathrm{C}}$ & $\begin{array}{l}\mathscr{n} \\
\stackrel{\mathscr{Z}}{丂}\end{array}$ & $\begin{array}{l}\text { ph } \\
\frac{0}{5} \\
\frac{5}{5}\end{array}$ & $\begin{array}{l}\stackrel{0}{0} \\
\frac{0}{5} \\
\end{array}$ & \begin{tabular}{l|l}
0 & $\frac{0}{5}$ \\
$\frac{5}{5}$ & $\frac{5}{5}$
\end{tabular} & 胥 & 总 & 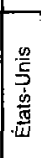 \\
\hline $\begin{array}{l}\text { 志 } \\
\stackrel{\sigma}{\sigma}\end{array}$ & 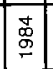 & 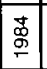 & 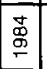 & $\begin{array}{l} \pm \\
0 \\
0 \\
-\end{array}$ & $\begin{array}{c}\text { 蒿 } \\
\text { : }\end{array}$ & $\mid \begin{array}{l} \pm \\
\text { 品 } \\
\sigma\end{array}$ & 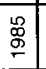 & 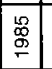 & $\begin{array}{l}\text { 吕 } \\
\text { 足 } \\
\end{array}$ & 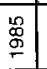 & $\begin{array}{l}\text { 邑 } \\
\stackrel{9}{\circ}\end{array}$ & $\mid \begin{array}{l}0 \\
0 \\
\sigma \\
\sigma\end{array}$ & \begin{tabular}{l|l}
$\stackrel{0}{0}$ \\
$\stackrel{0}{-}$
\end{tabular} & 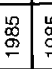 & 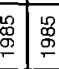 & \begin{tabular}{|l}
0 \\
$\stackrel{0}{\circ}$ \\
\end{tabular} & \begin{tabular}{|l|}
\multirow{2}{0}{} \\
$\stackrel{0}{\circ}$ \\
\end{tabular} & 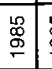 & \begin{tabular}{l|l}
$\stackrel{0}{0}$ \\
$\stackrel{0}{\sim}$ \\
\end{tabular} & 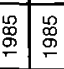 & \begin{tabular}{|l|}
$\mathscr{0}$ \\
$\mathbf{0}$ \\
\end{tabular} & \begin{tabular}{|l|}
\multirow{2}{\infty}{} \\
0 \\
0 \\
\end{tabular} & 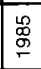 \\
\hline
\end{tabular}




\begin{tabular}{|c|c|c|c|c|c|c|c|c|c|c|c|c|c|c|c|c|c|c|c|c|c|c|c|c|}
\hline$\frac{9}{8}$ & 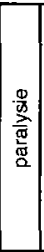 & 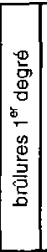 & $\sum_{\infty}$ & $\bar{\alpha}$ & & & 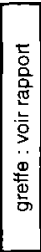 & & 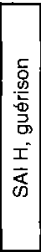 & $\frac{x}{\bar{x}}$ & 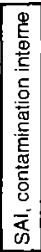 & & 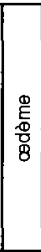 & 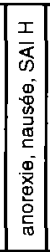 & 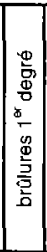 & 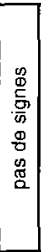 & 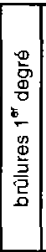 & 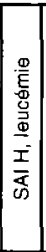 & & & & 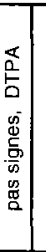 & & \\
\hline 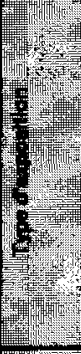 & 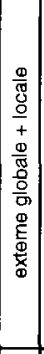 & 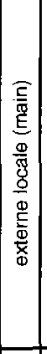 & 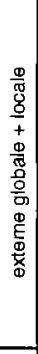 & 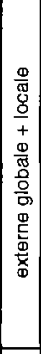 & 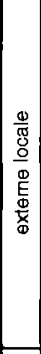 & 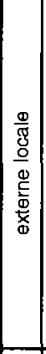 & 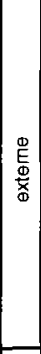 & 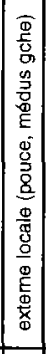 & 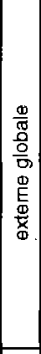 & 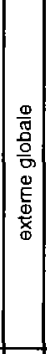 & 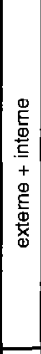 & 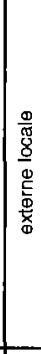 & 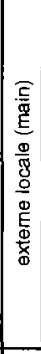 & 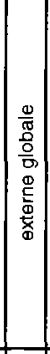 & 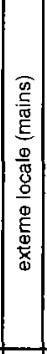 & 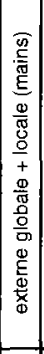 & 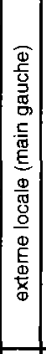 & 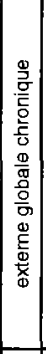 & & 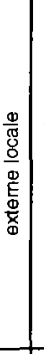 & 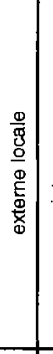 & $\begin{array}{l}\stackrel{9}{\mathrm{E}} \\
\stackrel{\mathrm{D}}{\mathrm{s}} \\
.\end{array}$ & 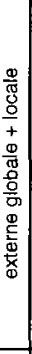 & 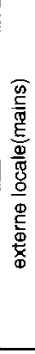 \\
\hline 48 & 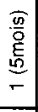 & $\circ$ & त् & - & 0 & 0 & $\stackrel{\mathscr{N}}{\mathbb{N}}$ & 0 & 0 & 0 & O & 0 & 0 & 0 & 0 & 0 & 0 & 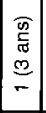 & 0. & $\circ$ & $\circ$ & 0 & 0 & 0 \\
\hline & $\sim$ & -1 & $\sigma$ & $\tau$ & - & - & $\begin{array}{l}: 0 \\
0 \\
\tilde{\sigma} \\
0\end{array}$ & - & $\sim$ & $\sim$ & 昌 & $r$ & - & $\begin{array}{c}\mathrm{v} \\
z \\
-\end{array}$ & $\sim$ & $\sigma$ & - & $r$ & $m$ & $\sim$ & - & $r$ & $\tau$ & $\infty$ \\
\hline 48 & 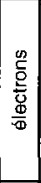 & 8 & 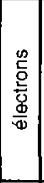 & $\stackrel{2}{2}$ & 8 & $=$ & $\mid \begin{array}{c}0 \\
0 \\
0 \\
0 \\
\frac{\omega}{2} \\
2\end{array}$ & $\frac{2}{2}$ & 8 & 8 & \begin{tabular}{|c|}
8 \\
8 \\
0 \\
0
\end{tabular} & $\underset{\Upsilon x}{\times}$ & $\underset{\widetilde{\alpha}}{x}$ & ${ }_{8}^{\circ}$ & x & 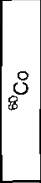 & $\underset{x}{x}$ & 8 & 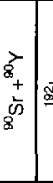 & $\frac{1}{20}$ & ড్ & $\sum_{\bar{L}}^{E}$ & $\frac{2}{2}$ & 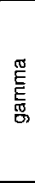 \\
\hline$\frac{1}{4}$ & 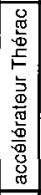 & 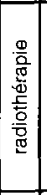 & 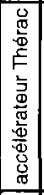 & & & 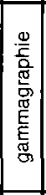 & 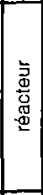 & & 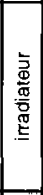 & 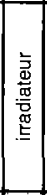 & 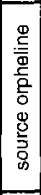 & & 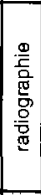 & 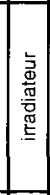 & 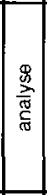 & 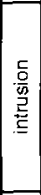 & $\begin{array}{l}\frac{0}{0} \\
\frac{2}{2} \\
\frac{\pi}{\pi} \\
\frac{\pi}{\pi}\end{array}$ & 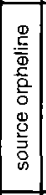 & & & & 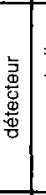 & 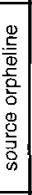 & 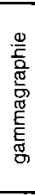 \\
\hline 0 & $\Sigma$ & $\Sigma$ & $\Sigma$ & - & - & - & 㕸 & - & - & - & $\Sigma$ & - & - & - & $x$ & $\Sigma$ & $\infty$ & - & a. & - & $\sim$ & - & - & - \\
\hline$H^{2}$ & 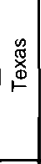 & & 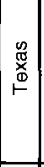 & & 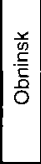 & 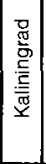 & 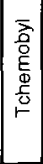 & 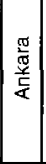 & 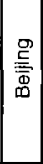 & 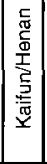 & $\begin{array}{l}\frac{\pi}{\underline{\underline{T}}} \\
\frac{\pi}{0} \\
0 \\
0\end{array}$ & 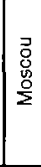 & 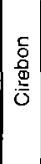 & 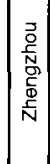 & 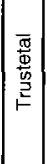 & 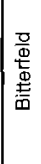 & $\begin{array}{l}\frac{\pi}{5} \\
\frac{5}{\omega}\end{array}$ & $\frac{\vec{z}}{\underline{x}}$ & 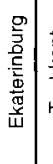 & 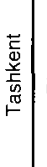 & & 造 & & 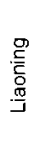 \\
\hline 8 & 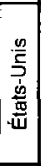 & 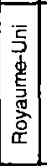 & 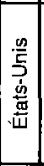 & $\begin{array}{l}\text { 욤 } \\
\text { 总 }\end{array}$ & 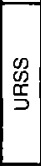 & $\begin{array}{l}0 \\
0 \\
\frac{0}{5} \\
\frac{0}{5}\end{array}$ & $\begin{array}{l}\mathscr{0} \\
\frac{0}{5} \\
\frac{1}{2}\end{array}$ & 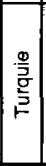 & $\begin{array}{l}2 \\
\text { : } \\
\end{array}$ & 苞 & 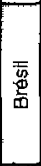 & $\begin{array}{l}\mathscr{y} \\
\frac{\pi}{5} \\
\end{array}$ & 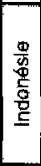 & 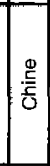 & ণ્વ & 总 & $\overleftarrow{\alpha}$ & $\begin{array}{l}0 \\
0 \\
0 \\
y \\
\end{array}$ & 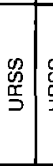 & 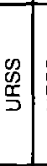 & 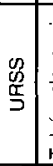 & 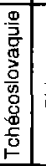 & 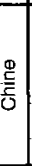 & $\frac{\mathscr{Q}}{\mathrm{E}}$ \\
\hline 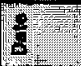 & \begin{tabular}{|l|}
0 \\
$\stackrel{0}{\leftarrow}$ \\
\end{tabular} & 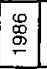 & 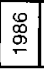 & $\begin{array}{l}0 \\
0 \\
0 \\
- \\
\end{array}$ & $\begin{array}{l}\stackrel{\circ}{\$} \\
\stackrel{2}{\circ} \\
\end{array}$ & 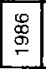 & 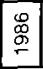 & \begin{tabular}{|l|} 
\\
$\stackrel{0}{\circ}$ \\
- \\
\end{tabular} & \begin{tabular}{|l|}
$\mathscr{0}$ \\
$\stackrel{0}{\circ}$ \\
\end{tabular} & \begin{tabular}{|l|}
$\stackrel{0}{8}$ \\
$\stackrel{0}{\circ}$ \\
\end{tabular} & \begin{tabular}{|l}
0 \\
0 \\
0 \\
\hdashline
\end{tabular} & \begin{tabular}{|l}
$\stackrel{a}{\infty}$ \\
$\stackrel{5}{\circ}$
\end{tabular} & $\stackrel{\circ}{\circ}$ & \begin{tabular}{|l|}
$\hat{\mathbf{D}}$ \\
$\mathbf{0}$ \\
\end{tabular} & \begin{tabular}{|l|}
0 \\
0 \\
0 \\
0
\end{tabular} & 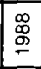 & \begin{tabular}{|l|} 
\\
0 \\
0 \\
0 \\
\end{tabular} & \begin{tabular}{|l|}
0 \\
0 \\
0 \\
0 \\
\end{tabular} & \begin{tabular}{c|c}
$\stackrel{0}{0}$ \\
$\stackrel{8}{-}$
\end{tabular} & 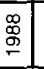 & \begin{tabular}{|l|l|l}
\multirow{2}{*}{} & \\
$\stackrel{8}{\circ}$ & \\
\end{tabular} & 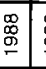 & $\begin{array}{l}\mathbf{0} \\
0 \\
0 \\
0\end{array}$ & 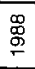 \\
\hline
\end{tabular}




\begin{tabular}{|c|c|c|c|c|c|c|c|c|c|c|c|c|c|c|c|c|c|c|c|c|c|c|c|c|c|}
\hline 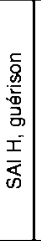 & 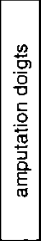 & 8 & $\mid \begin{array}{l}\mathbf{a} \\
\vdots \\
\vdots\end{array}$ & & & & & 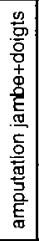 & & & 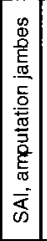 & & 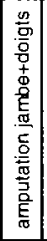 & 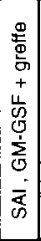 & & & & 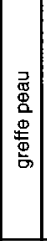 & & & 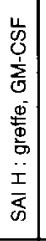 & & & $\overline{\tilde{\sigma}}$ & 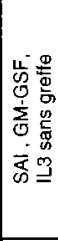 \\
\hline 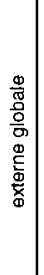 & 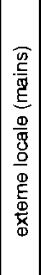 & 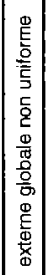 & 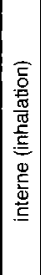 & 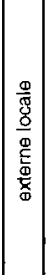 & 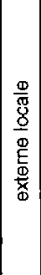 & 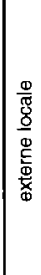 & 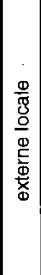 & 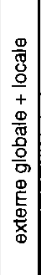 & 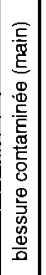 & 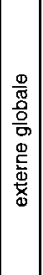 & 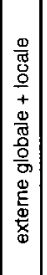 & 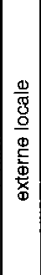 & 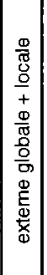 & 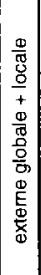 & 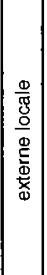 & 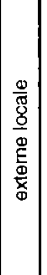 & 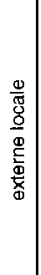 & 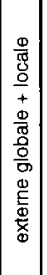 & 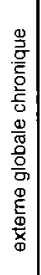 & 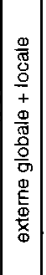 & 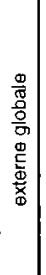 & 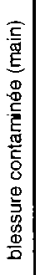 & 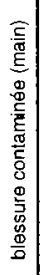 & 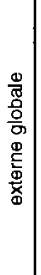 & 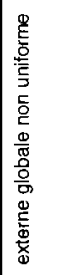 \\
\hline 0 & 0 & 0 & 0 & 0 & 0 & 0 & 0 & 0 & 0 & 0 & $\begin{array}{c}\stackrel{=}{\hat{0}} \\
\stackrel{5}{=}\end{array}$ & 0 & 0 & $\frac{c}{0}$ & 0 & 0 & 0 & 0 & 0 & $\frac{m}{1}$ & 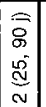 & 0 & 0 & 0 & $\stackrel{\stackrel{0}{\underline{m}}}{\underline{\underline{c}}}$ \\
\hline- & - & - & - & - & - & - & - & $\infty$ & - & - & m & - & $\infty$ & - & - & - & - & - & m & $\hat{\sim}$ & 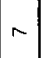 & - & r & $\infty$ & - \\
\hline 8 & $\underset{\mathscr{\Phi}}{\stackrel{2}{*}}$ & & $\frac{\frac{E}{4}}{\bar{a}}$ & 뜸 & 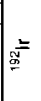 & \begin{tabular}{|l}
$\frac{2}{5}$ \\
$\frac{5}{E}$ \\
$\frac{\tilde{\omega}}{\omega}$
\end{tabular} & 또 & $=$ & 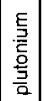 & $=$ & 8 & $\stackrel{*}{\%}$ & 8 & $\ddot{0}$ & $\underset{\Phi}{2}$ & 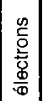 & $=$ & $\stackrel{=}{\Phi}$ & $\stackrel{\infty}{0}$ & & ¿ & $\begin{array}{l}E \\
\frac{E}{4} \\
\dot{3}\end{array}$ & 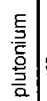 & $\stackrel{\infty}{0}$ & 8 \\
\hline 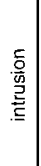 & 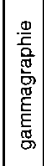 & 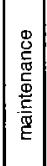 & & & 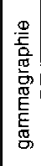 & 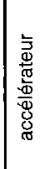 & & 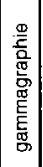 & & & 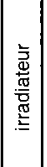 & 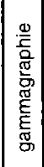 & 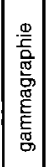 & 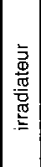 & 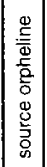 & 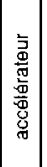 & 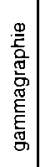 & 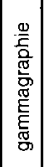 & & 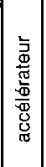 & 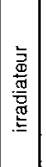 & & 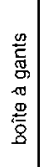 & $\stackrel{\text { g }}{\mathrm{g}}$ & 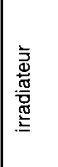 \\
\hline- & - & 0 & $\frac{0}{z}$ & - & - & $a$ & - & - & $\mp$ & - & - & - & - & - & - & $a$ & - & - & - & $\Sigma$ & - & $\bar{\Sigma}$ & $\stackrel{0}{2}$ & $\cdots$ & - \\
\hline 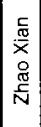 & \begin{tabular}{|l}
$\frac{\pi}{\pi}$ \\
$\frac{\tilde{m}}{\mathbf{T}}$ \\
\end{tabular} & 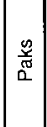 & & $\begin{array}{l}\vec{\partial} \\
0 \\
\stackrel{0}{\Sigma} \\
\Sigma\end{array}$ & $\cdots$ & 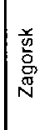 & 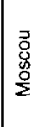 & 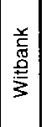 & 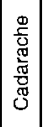 & & 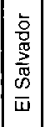 & & 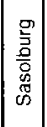 & $\begin{array}{l}\bar{\sigma} \\
\bar{\Phi} \\
\overline{0} \\
\end{array}$ & 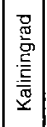 & 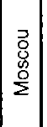 & 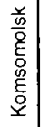 & $\mid \begin{array}{l}\vec{z} \\
\frac{\vec{c}}{\bar{v}} \\
\frac{w}{y} \\
\end{array}$ & $\begin{array}{l}\frac{\pi}{0} \\
\frac{3}{3} \\
\frac{3}{4}\end{array}$ & 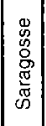 & 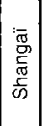 & 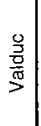 & 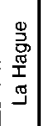 & 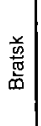 & 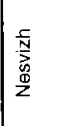 \\
\hline 默 & 要 & \begin{tabular}{|l} 
D \\
5 \\
$\mathbf{0}$ \\
$\mathbf{5}$ \\
\end{tabular} & 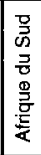 & $\begin{array}{l}\mathscr{y} \\
0 \\
\frac{0}{\partial} \\
\end{array}$ & $\begin{array}{l}\text { D } \\
\text { D) } \\
\text { 年 }\end{array}$ & 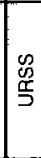 & \begin{tabular}{|l}
0 \\
0 \\
0 \\
$\frac{5}{5}$
\end{tabular} & 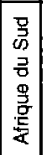 & 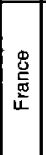 & 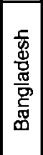 & 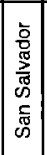 & $\frac{\text { ge }}{\frac{5}{0}}$ & 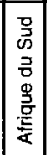 & 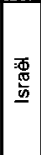 & $\mid \begin{array}{l}0 \\
0 \\
\tilde{\sigma} \\
\end{array}$ & $\mid \begin{array}{l}0 \\
0 \\
\frac{1}{5}\end{array}$ & 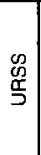 & $\mid \begin{array}{l}\infty \\
0 \\
\underline{\underline{I}}\end{array}$ & 든 & 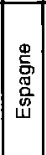 & 焉 & 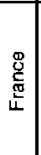 & $\begin{array}{l}\mathbb{q} \\
\stackrel{\mathbb{v}}{4} \\
\end{array}$ & 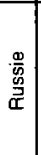 & 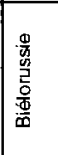 \\
\hline 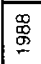 & 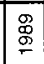 & 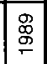 & 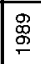 & 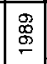 & 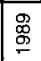 & $\begin{array}{l}\text { g̊ } \\
\text { 学 }\end{array}$ & 棁 & \begin{tabular}{|l}
$g$ \\
8 \\
\end{tabular} & 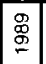 & 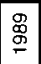 & 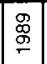 & 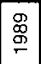 & $\begin{array}{l} \\
\\
\end{array}$ & 兽 & $\begin{array}{l}\text { 品 } \\
\end{array}$ & $\begin{array}{l} \\
口 \\
\end{array}$ & \begin{tabular}{|l|} 
\\
\end{tabular} & \begin{tabular}{|l|} 
\\
$\stackrel{S}{\sigma}$ \\
\end{tabular} & 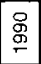 & $\begin{array}{l}\stackrel{\circ}{\circ} \\
\stackrel{\circ}{\circ}\end{array}$ & $\underset{8}{\stackrel{\circ}{\circ}}$ & $\begin{array}{l}\bar{\Phi} \\
\end{array}$ & $\stackrel{.}{\circ}$ & 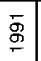 & g. \\
\hline
\end{tabular}




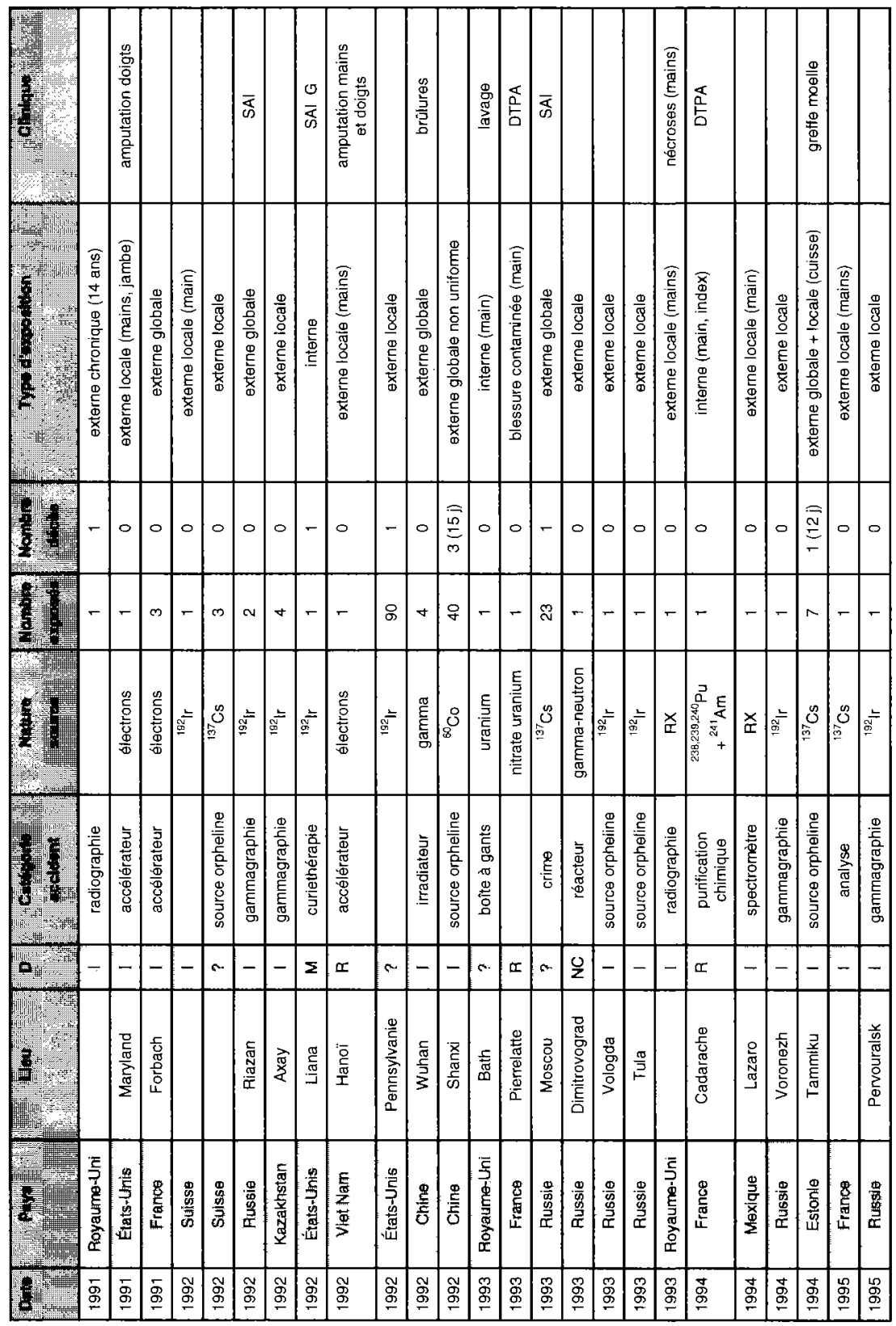




\begin{tabular}{|c|c|c|c|c|c|c|c|c|c|c|c|c|c|c|c|c|c|c|c|c|c|c|c|c|}
\hline & 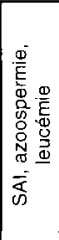 & & & & 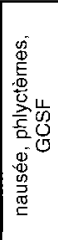 & & 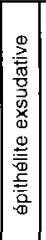 & & & 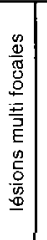 & 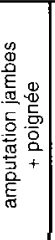 & & & & 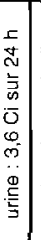 & 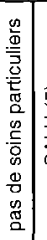 & 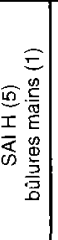 & & 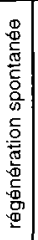 & 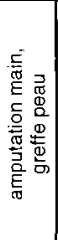 & & 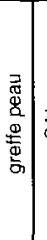 & $\bar{a}$ & 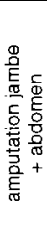 \\
\hline 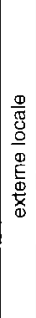 & 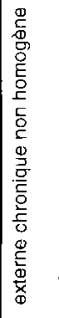 & 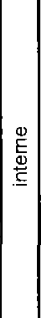 & 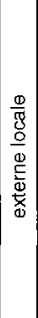 & 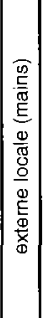 & 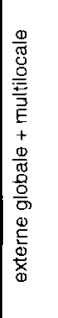 & 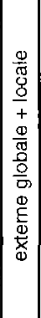 & 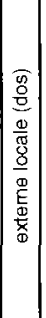 & 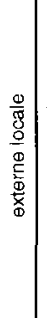 & 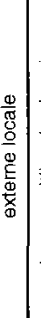 & 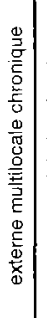 & 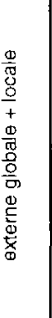 & 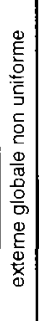 & 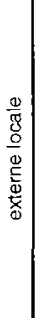 & 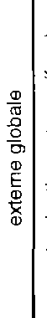 & 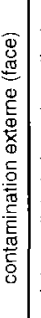 & 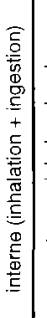 & 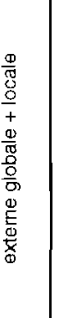 & 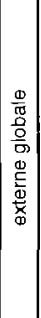 & 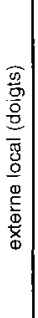 & 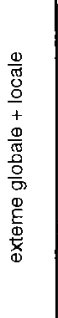 & 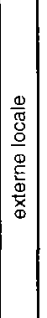 & 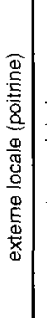 & 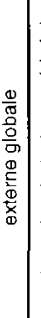 & 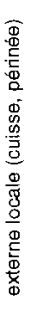 \\
\hline 0 & 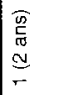 & $\circ$ & 0 & 0 & 0 & $\begin{array}{l}+ \\
m \\
m\end{array}$ & 0 & $\circ$ & 0 & 0 & 0 & $\stackrel{2}{2}$ & 0 & 0 & 0 & 0 & 0 & 0 & 0 & r. & 0 & 0 & - & 0 \\
\hline- & - & - & - & - & - & $\stackrel{\vec{t}}{\check{r}}$ & - & - & - & $F$ & $r$ & - & - & $\sim$ & - & - & $\stackrel{\infty}{\leftarrow}$ & $\div$ & - & $\omega$ & - & - & c. & $\infty$ \\
\hline$\frac{2}{w^{2}}$ & 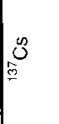 & 10 & $\stackrel{2}{\underline{2}}$ & 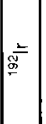 & 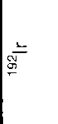 & 8 & $\stackrel{x}{x}$ & 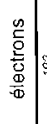 & $=$ & 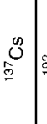 & $\frac{2}{2}$ & 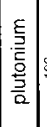 & 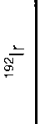 & 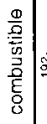 & 한 & $\begin{array}{c}\frac{E}{\alpha} \\
\vdots \\
\vdots \\
+ \\
\vec{J} \\
\frac{0}{5}\end{array}$ & 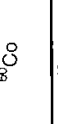 & 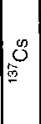 & 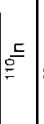 & $\begin{array}{l}\circ \\
8\end{array}$ & $\begin{array}{c}8 \\
8\end{array}$ & 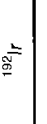 & 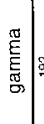 & 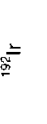 \\
\hline 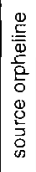 & 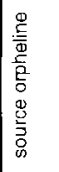 & 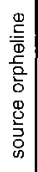 & 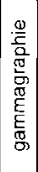 & 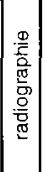 & 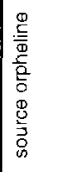 & 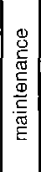 & 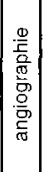 & 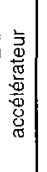 & 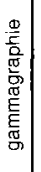 & 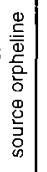 & 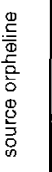 & 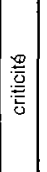 & 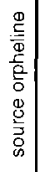 & 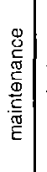 & 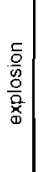 & 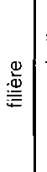 & 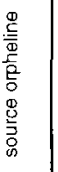 & 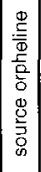 & 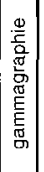 & 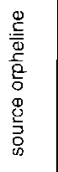 & 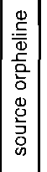 & 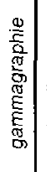 & 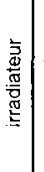 & 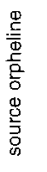 \\
\hline c. & - & 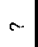 & - & $\Sigma$ & - & $\Sigma$ & $\Sigma$ & a. & - & $\bar{\Sigma}$ & - & $\bar{\Sigma}$ & - & :0 & - & $\stackrel{0}{Z}$ & $\Sigma$ & $\bar{\Sigma}$ & $\pi$ & - & $\cdots$ & - & - & - \\
\hline 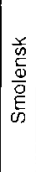 & 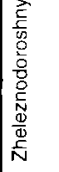 & \begin{tabular}{|l|}
$\overrightarrow{0}$ \\
0 \\
0 \\
$\Sigma$ \\
$\Sigma$ \\
\end{tabular} & $\frac{\vec{c}}{2}$ & & $\frac{\frac{c}{\sigma}}{\bar{\sigma}}$ & 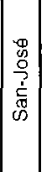 & $\mid \begin{array}{l}0 \\
0 \\
0 \\
0 \\
0 \\
3 \\
\end{array}$ & 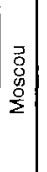 & 窟 & $\stackrel{\circ}{\Xi}$ & 涉 & 总 & $\begin{array}{l}\overline{0} \\
0 \\
0 \\
\frac{0}{5} \\
\end{array}$ & 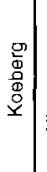 & 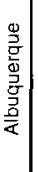 & 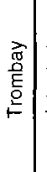 & $\begin{array}{l}\overline{\bar{z}} \\
\text { 喜 } \\
\underline{\underline{w}} \\
\underline{\underline{m}}\end{array}$ & 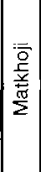 & 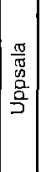 & $\begin{array}{l}\text { ते } \\
\text { o } \\
\text { : }\end{array}$ & $\begin{array}{l}\vec{z} \\
\bar{o} \\
\stackrel{0}{\Sigma} \\
\end{array}$ & $\begin{array}{l}9 \\
8 \\
0 \\
9 \\
-1\end{array}$ & & 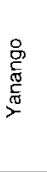 \\
\hline $\begin{array}{l}\frac{\Phi}{\omega} \\
\underline{\underline{\omega}} \\
\widetilde{\vec{\sigma}}\end{array}$ & 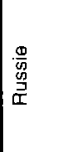 & 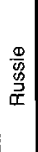 & $\mid \begin{array}{l}\frac{\boldsymbol{w}}{w} \\
\vec{w} \\
\overrightarrow{\widetilde{x}}\end{array}$ & 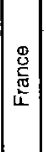 & 듬 & $\mid \begin{array}{l}\frac{\mathrm{g}}{\sigma} \\
\frac{\mathrm{g}}{\mathrm{w}} \\
\mathrm{g} \\
0\end{array}$ & 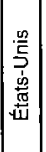 & $\mid \begin{array}{c}\frac{\Phi}{\omega} \\
\vec{\omega} \\
\vec{\omega} \\
\end{array}$ & 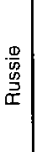 & $\begin{array}{l}0 \\
0 \\
0 \\
0 \\
0\end{array}$ & 趇 & 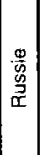 & 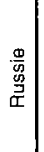 & 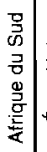 & 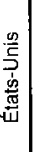 & $\begin{array}{l}\stackrel{\mathbb{D}}{\mathrm{g}} \\
\underline{\underline{F}}\end{array}$ & 墨 & 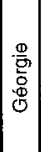 & 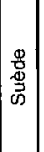 & 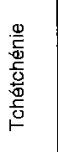 & 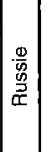 & 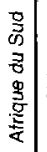 & 畩 & 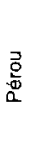 \\
\hline 昭 & 兽 & $\mid$\begin{tabular}{l} 
另 \\
\hdashline \\
\hdashline
\end{tabular} & 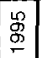 & 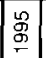 & 兽 & 兽 & $\mid \begin{array}{l}\stackrel{g}{\stackrel{g}{\sigma}} \\
\end{array}$ & $\mid \begin{array}{l}\mathscr{g} \\
\stackrel{\circ}{\circ}\end{array}$ & 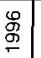 & $\mid \begin{array}{l}0 \\
0 \\
0 \\
0\end{array}$ & 棁 & $\mid \begin{array}{c}\mathrm{g} \\
\end{array}$ & $\mid \begin{array}{l}\hat{\circ} \\
\text { \% }\end{array}$ & 句 & $\begin{array}{l}5 \\
\end{array}$ & 稃 & 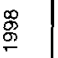 & 兽 & $\mid$\begin{tabular}{l}
$\infty$ \\
0 \\
0 \\
\hdashline
\end{tabular} & $\begin{array}{l}\stackrel{g}{8} \\
\stackrel{8}{\circ}\end{array}$ & $\begin{array}{l}\stackrel{\infty}{\%} \\
\stackrel{\sigma}{\sigma}\end{array}$ & $\begin{array}{l}\text { 怘 } \\
\stackrel{2}{2}\end{array}$ & $\stackrel{\infty}{\stackrel{\Xi}{\sigma}}$ & 总 \\
\hline
\end{tabular}




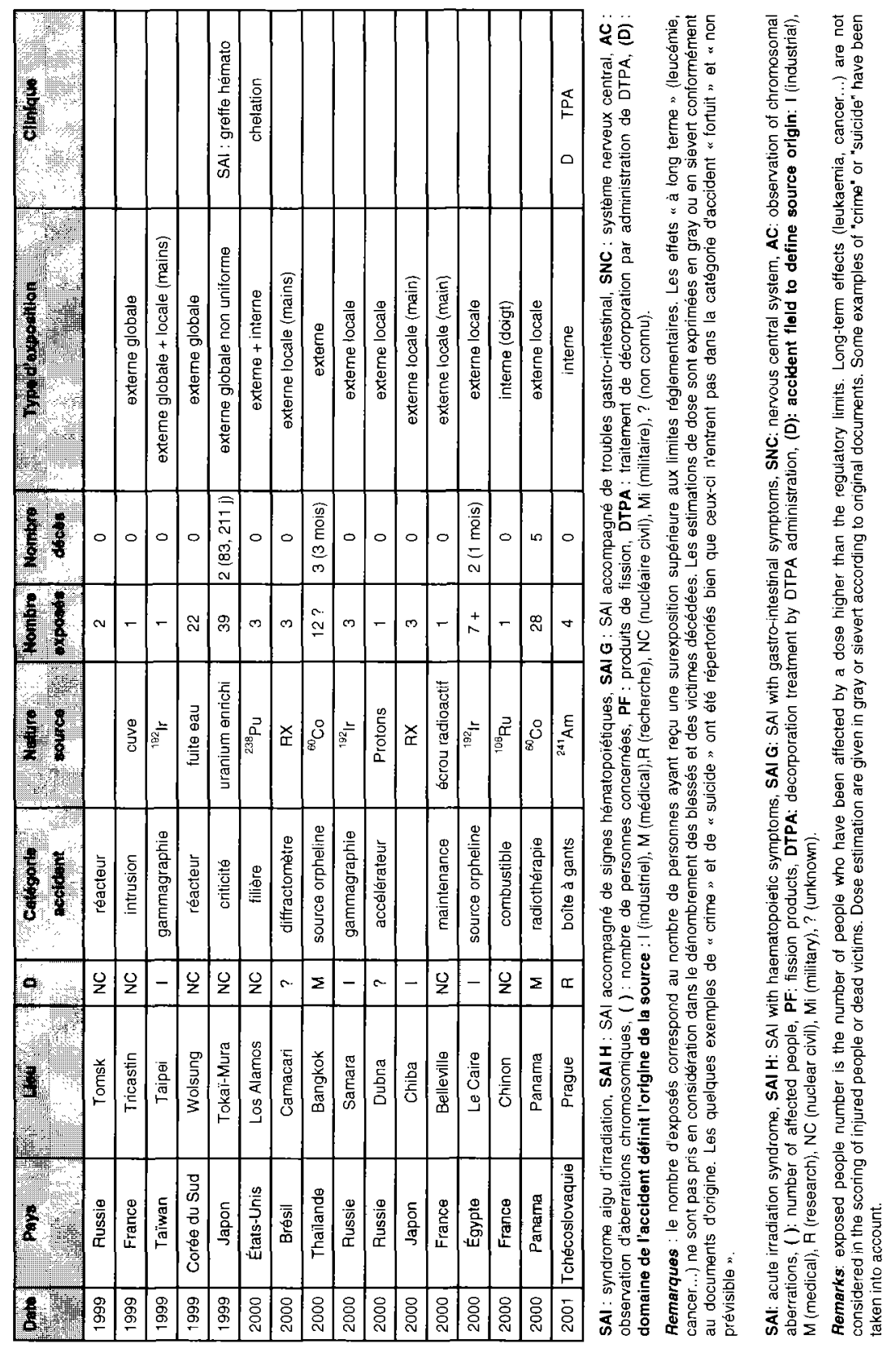

